\title{
REAL-VARIABLE CHARACTERIZATIONS OF MUSIELAK-ORLICZ HARDY SPACES ON SPACES OF HOMOGENEOUS TYPE
}

\author{
Xing Fu, Tao Ma and Dachun Yang* \\ Hubei University, Faculty of Mathematics and Statistics \\ Hubei Key Laboratory of Applied Mathematics \\ Wuhan 430062, P. R. China; xingfu@hubu.edu.cn \\ Wuhan University, School of Mathematics and Statistics \\ Wuhan 430072, P. R. China; tma.math@whu.edu.cn \\ Beijing Normal University, School of Mathematical Sciences \\ Laboratory of Mathematics and Complex Systems (Ministry of Education of China) \\ Beijing 100875, P. R. China; dcyang@bnu.edu.cn
}

\begin{abstract}
Let $(\mathcal{X}, d, \mu)$ be a space of homogeneous type in the sense of Coifman and Weiss. In this article, the authors establish a complete real-variable theory of Musielak-Orlicz Hardy spaces on $(\mathcal{X}, d, \mu)$. To be precise, the authors first introduce the atomic Musielak-Orlicz Hardy space $H_{\text {at }}^{\varphi}(\mathcal{X})$ and then establish its various maximal function characterizations. The authors also investigate the Littlewood-Paley characterizations of $H_{\mathrm{at}}^{\varphi}(\mathcal{X})$ via Lusin area functions, LittlewoodPaley $g$-functions and Littlewood-Paley $g_{\lambda}^{*}$-functions. The authors further obtain the finite atomic characterization of $H_{\mathrm{at}}^{\varphi}(\mathcal{X})$ and its improved version in case $q<\infty$, and their applications to criteria of the boundedness of sublinear operators from $H_{\mathrm{at}}^{\varphi}(\mathcal{X})$ to a quasi-Banach space, which are also applied to the boundedness of Calderón-Zygmund operators. Moreover, the authors find the dual space of $H_{\mathrm{at}}^{\varphi}(\mathcal{X})$, namely, the Musielak-Orlicz $\mathrm{BMO}$ space $\mathrm{BMO}^{\varphi}(\mathcal{X})$, present its several equivalent characterizations, and apply it to establish a new characterization of the set of pointwise multipliers for the space $\operatorname{BMO}(\mathcal{X})$. The main novelty of this article is that, throughout the article, except the last section, $\mu$ is not assumed to satisfy the reverse doubling condition.
\end{abstract}

\section{Introduction}

The classical real Hardy space $H^{p}\left(\mathbf{R}^{n}\right)$ on the $n$-dimensional Euclidean space has proved a nice substitute of the Lebesgue space $L^{p}\left(\mathbf{R}^{n}\right)$ when $p \in(0,1]$, because some important operators (for instance, the Riesz transforms) are not bounded on $L^{p}\left(\mathbf{R}^{n}\right)$ but bounded on $H^{p}\left(\mathbf{R}^{n}\right)$ when $p \in(0,1]$. The real-variable theory of $H^{p}\left(\mathbf{R}^{n}\right)$ was initiated by Stein and Weiss [57] and later extensively developed by Fefferman and Stein [13]. Precisely, Fefferman and Stein [13] established various maximal function characterizations of $H^{p}\left(\mathbf{R}^{n}\right)$ and found a surprising result on the dual space of $H^{1}\left(\mathbf{R}^{n}\right)$, which is just $\operatorname{BMO}\left(\mathbf{R}^{n}\right)$, the space of functions with bounded mean oscillations introduced by John and Nirenberg [33]. To better understand the structure

\footnotetext{
https://doi.org/10.5186/aasfm.2020.4519

2010 Mathematics Subject Classification: Primary 42B30; Secondary 42B25, 42B20, 42B35, 30L99.

Key words: Space of homogeneous type, Musielak-Orlicz Hardy space, atom, sublinear operator, pointwise multiplier.

This project is supported by the National Natural Science Foundation of China (Grant Nos. 11701160, 11871100, 11671308, 11431011, 11971058, 11761131002 and 11671185) and the independent research project of Wuhan University (Grant No. 2042017kf0209).

*Corresponding author.
} 
of Hardy spaces, one finds that a core idea is to decompose any element of $H^{p}\left(\mathbf{R}^{n}\right)$ into a sum of some basic elements with some good properties, which are called atoms (see Coifman [8] for the case $n=1$ and Latter [39] for the general case $n \in \mathbf{N}$ ). This paves the way for the extensive study of the boundedness on Hardy spaces of many classical operators appearing in harmonic analysis such as Calderón-Zygmund operators and fractional integrals. For more studies on the real-variable theory of Hardy spaces and its applications to harmonic analysis and partial differential equations, we refer the reader to some fundamental references, for instance, [57, 55, 13, 56, 48].

In the last several decades, the classical real Hardy spaces were generalized to other type Hardy spaces. Among this, Ky [35] extended both the Orlicz-Hardy spaces and the weighted Hardy spaces to new Orlicz type Hardy spaces, Musielak-Orlicz Hardy spaces $H^{\varphi}\left(\mathbf{R}^{n}\right)$, which prove useful. Particularly, the Musielak-Orlicz Hardy space $H^{\log }\left(\mathbf{R}^{n}\right)$, related to the typical growth function

$$
\theta(x, t):=\frac{t}{\log (e+|x|)+\log (e+t)}, \quad \forall x \in \mathbf{R}^{n}, \forall t \in[0, \infty),
$$

plays essential roles in the bilinear decompositions of $\operatorname{BMO}\left(\mathbf{R}^{n}\right) \times H^{1}\left(\mathbf{R}^{n}\right)$. These bilinear decompositions are important to the optimal endpoint estimate for the divcurl lemma, involving the space $H^{\log }\left(\mathbf{R}^{n}\right)$, and have also relations to an implicit conjecture from [6] (see also [5, 4]). These bilinear decompositions also play striking roles in bilinear or subbilinear decompositions, respectively, for linear or sublinear commutators of Calderón-Zygmund operators from [34]; see [36, 37] (or [59]) for more applications. So far, some literature on the real-variable theory of $H^{\varphi}\left(\mathbf{R}^{n}\right)$ and its applications appeared (see, for instance, [30, 35, 41, 42, 43, 44, 59, 60]).

To extend some classical results of harmonic analysis over Euclidean spaces to more generalized setting, Coifman and Weiss $[9,10]$ introduced the following notion of the space of homogeneous type, which is a natural setting for the theory of functions spaces and operators in harmonic analysis. Let us first recall the following notion of spaces of homogeneous type from $[9,10]$. Suppose that $\mathcal{X}$ is a non-empty set equipped with a quasi-metric $d$ with the quasi-triangle constant $A_{0} \in[1, \infty)$, namely, for any $x, y, z \in \mathcal{X}$,

(i) $d(x, y)=d(y, x)$;

(ii) $d(x, y)=0$ if and only if $x=y$;

(iii) $d$ satisfies the quasi-triangle inequality

$$
d(x, y) \leq A_{0}[d(x, z)+d(z, y)] .
$$

The triple $(\mathcal{X}, d, \mu)$ is called a space of homogeneous type if $\mu$ is a non-negative measure satisfying the doubling condition: there exists a positive constant $C_{(\mathcal{X})} \in$ $[1, \infty)$ such that, for any ball $B(x, r):=\{y \in \mathcal{X}: d(x, y)<r\}$ with $(x, r) \in \mathcal{X} \times$ $(0, \infty)$,

$$
\mu(B(x, 2 r)) \leq C_{(\mathcal{X})} \mu(B(x, r)),
$$

which further implies that there exists a positive constant $\widetilde{C}_{(\mathcal{X})}$ such that, for any $\lambda \in[1, \infty)$ and $(x, r) \in \mathcal{X} \times(0, \infty)$,

$$
\mu(B(x, \lambda r)) \leq \widetilde{C}_{(\mathcal{X})} \lambda^{\omega} \mu(B(x, r)),
$$

where $\omega:=\log _{2} C_{(\mathcal{X})}$.

Remark 1.1. Two typical examples of spaces of homogeneous type are as follows. 
(i) Let $\mathcal{X}:=\mathbf{R}^{n}, d(x, y):=\left[\sum_{j=1}^{n}\left(x_{i}-y_{i}\right)^{2}\right]^{1 / 2}$ for any $x:=\left(x_{1}, \ldots, x_{n}\right), y:=$ $\left(y_{1}, \ldots, y_{n}\right) \in \mathbf{R}^{n}$, and $\mu$ be the $n$-dimensional Lebesgue measure. This is just the Euclidean space equipped with the $n$-dimensional Lebesgue measure.

(ii) Let $\mathcal{X}:=\mathbf{R}^{n}, d(x, y):=\sum_{j=1}^{n}\left|x_{i}-y_{i}\right|^{\alpha_{i}}$ for any $x:=\left(x_{1}, \ldots, x_{n}\right), y:=$ $\left(y_{1}, \ldots, y_{n}\right) \in \mathbf{R}^{n}$, where $\alpha_{1}, \ldots, \alpha_{n} \in(0, \infty)$ are not all equal, and $\mu$ be the $n$-dimensional Lebesgue measure. This is so called the anisotropic Euclidean space equipped with the $n$-dimensional Lebesgue measure.

Please see [10, pp. 588-590] for more examples of spaces of homogeneous type.

In this setting, Coifman and Weiss [10] introduced the atomic Hardy space $H_{\mathrm{at}}^{p, q}(\mathcal{X})$ and proved that $H_{\mathrm{at}}^{p, q}(\mathcal{X})$ is independent of the choice of $q$. Hereafter, $H_{\mathrm{at}}^{p, q}(\mathcal{X})$ is simply denoted by $H_{\mathrm{at}}^{p}(\mathcal{X})$. Coifman and Weiss [10] also showed that its dual space is the Lipschitz space $\operatorname{Lip}_{1 / p-1}(\mathcal{X})$ when $p \in(0,1)$, or the space $\operatorname{BMO}(\mathcal{X})$ when $p=1$.

However, some important tools in the real-variable theory of $H_{\mathrm{at}}^{p}(\mathcal{X})$, such as the Calderón reproducing formulae, seem to need additional assumptions. To this end, Han et al. [26] (see also [25]) introduced the RD-space, namely, the space of homogeneous type satisfying the following reverse doubling condition: there exist some positive constants $a_{0}, c_{(\mathcal{X})} \in(1, \infty)$ such that, for any $x \in \mathcal{X}$ and $r \in\left(0, \operatorname{diam}(\mathcal{X}) / a_{0}\right)$ with $\operatorname{diam}(\mathcal{X}):=\sup \{d(x, y): x, y \in \mathcal{X}\}$,

$$
\mu\left(B\left(x, a_{0} r\right)\right) \geq c_{(\mathcal{X})} \mu(B(x, r)) ;
$$

see [62] for several equivalent characterizations of the reverse doubling condition (1.5). Later, a real-variable theory of various function spaces and their applications were given on RD-spaces; see, for instance, [25, 26, 61, 62, 21] and the references therein.

Recently, motivated by the remarkable wavelet theory of Auscher and Hytönen in [2], Han et al. [23, 24] established a partial real-variable theory of (product) Hardy spaces on spaces of homogeneous type without having recourse to the assumption (1.5), via using the orthonormal basis of regular wavelets from $[2,3]$ and investigating its applications to the boundedness of Calderón-Zygmund operators. Meanwhile, Fu et al. [19] confirmed a conjecture raised by Bonami and Bernicot (see Ky [38, p. 809]) that $f \times g$ of $f \in H_{\text {at }}^{1}(\mathcal{X})$ and $g \in \operatorname{BMO}(\mathcal{X})$ can be written into a sum of two bilinear operators on any metric measure space of homogeneous type without having recourse to the reverse doubling condition (1.5), where several equivalent wavelet characterizations of $H_{\mathrm{at}}^{1}(\mathcal{X})$ from [17] and a typical Musielak-Orlicz Hardy space $H^{\log }(\mathcal{X})$ play crucial roles. The space $H^{\log }(\mathcal{X})$ is also important to the local version of the above bilinear decomposition in [16]. Moreover, the bilinear decomposition in [19] is also useful to the endpoint boundedness of the (sub-)linear commutator $[b, T]$ of a sublinear operator $T$ and $b \in \operatorname{BMO}(\mathcal{X})$ on Hardy spaces in [46, 47]; see the survey [15] for more details.

More recently, He et al. [28] constructed a corresponding wavelet reproducing formulae without having recourse to the reverse doubling condition (1.5). Based on these wavelet reproducing formulae, $\mathrm{He}$ et al. [27] further established a complete real-variable theory of Hardy spaces $H^{p}(\mathcal{X})$ on spaces of homogeneous type without having recourse to the reverse doubling condition (1.5). On another hand, Hou et al. [31] investigated Musielak-Orlicz BMO-type spaces associated with generalized approximations to the identity on spaces of homogeneous type.

Motivated by [27] and the useful space $H^{\log }(\mathcal{X})$, one naturally expects to develop a complete real-variable theory of Musielak-Orlicz Hardy spaces, including the above 
useful space $H^{\log }(\mathcal{X})$. In this article, we positively answer this question on $(\mathcal{X}, d, \mu)$. Precisely, we introduce the atomic Musielak-Orlicz Hardy space $H_{\text {at }}^{\varphi}(\mathcal{X})$ and then investigate their various maximal function characterizations, the Littlewood-Paley characterizations, the finite atomic characterizations and the dual space of $H_{\mathrm{at}}^{\varphi}(\mathcal{X})$. We also consider their applications to the boundedness of sublinear operators, including Calderón-Zygmund operators as concrete instances, from $H_{\text {at }}^{\varphi}(\mathcal{X})$ to a quasiBanach space, and a characterization of the set of pointwise multipliers for the space $\operatorname{BMO}(\mathcal{X})$. It should be pointed out that the main novelty of this article is that, except Section 10, $\mu$ is not assumed to satisfy the reverse doubling condition (1.5).

Throughout this article, but except the last section, we always assume that $(\mathcal{X}, d, \mu)$ is a space of homogeneous type, which is non-atomic [namely, $\mu(\{x\})=0$ for any $x \in \mathcal{X}]$ and satisfies $\operatorname{diam}(\mathcal{X})=\infty$. It is known that $\operatorname{diam}(\mathcal{X})=\infty$ implies that $\mu(\mathcal{X})=\infty$ (see, for instance, [53, Lemma 5.1] or [2, Lemma 8.1]).

The organization of this article is as follows.

In Section 2, we recall some basic notions, notation and known results used in this article.

In Section 3, by establishing a version of Calderón-Zygmund decompositions on balls and borrowing some ideas from the proof of [43, Theorem 2.5], we obtain the John-Nirenberg inequality of $\mathrm{BMO}^{\varphi}(\mathcal{X})$ (see Theorem 3.1 below). Then we apply this John-Nirenberg inequality to establish several equivalent characterizations for $\mathrm{BMO}^{\varphi}(\mathcal{X})$, which are similar to [43, Theorem 2.7] with some technical modifications.

In Section 4, we first recall the important Calderón reproducing formulae established in [27] and then introduce Musielak-Orlicz Hardy spaces, $H^{+, \varphi}(\mathcal{X}), H_{\theta}^{\varphi}(\mathcal{X})$ and $H^{*, \varphi}(\mathcal{X})$ defined, respectively, via radial, nontangential and grand maximal functions. In Subsection 4.2 , by the pointwise inequalities for those maximal functions and some ideas from the proof of [27, Theorem 3.5], we show that $H^{+, \varphi}(\mathcal{X}), H_{\theta}^{\varphi}(\mathcal{X})$ and $H^{*, \varphi}(\mathcal{X})$ are mutually identical with equivalent quasi-norms (see Theorem 4.12 below).

Section 5 is devoted to the atomic characterizations of $H^{*, \varphi}(X)$ (see Theorem 5.4 below). The proof is divided into three parts. In Subsection 5.1, we show $H_{\mathrm{at}}^{\varphi, q}(\mathcal{X}) \subset$ $H^{*, \varphi}(\mathcal{X})$ via the properties of the growth functions $\varphi$ and some arguments used in the proof of [35, Theorem 4.2]. Then, in Subsection 5.2, we obtain a version of Calderón-Zygmund decompositions for $H^{*, \varphi}(\mathcal{X})$ (see Proposition 5.8 below), which is a generalization of the corresponding results on $H^{p}(\mathcal{X})$ in [27, Proposition 4.9]. In Subsection 5.3, via this Calderón-Zygmund decompositions for $H^{*, \varphi}(\mathcal{X})$, a technical lemma on the density of $L_{\varphi(\cdot, 1)}^{q}(\mathcal{X}) \cap H^{*, \varphi}(\mathcal{X})$ in $H^{*, \varphi}(\mathcal{X})$ and some arguments similar to those used in the proof of [27, Theorem 4.2], we prove that $H^{*, \varphi}(\mathcal{X}) \subset H_{\mathrm{at}}^{\varphi, q}(\mathcal{X})$, namely, we establish the atomic decomposition of $H^{*, \varphi}(\mathcal{X})$.

In Section 6, we study the Littlewood-Paley characterizations of $H_{\mathrm{at}}^{\varphi}(\mathcal{X})$, respectively, in terms of Lusin area functions, Littlewood-Paley $g$-functions and LittlewoodPaley $g_{\lambda}^{*}$-functions. Precisely, in Subsection 6.1, via the vector-valued FeffermanStein maximal inequality, we first show the independence of the choices of exp-ATIs on the Musielak-Orlicz Hardy space $H^{\varphi}(\mathcal{X})$ defined by the Lusin area functions (see Theorem 6.3 below). Then we obtain the molecular characterizations of $H^{\varphi}(\mathcal{X})$ in Subsection 6.2 (see Theorem 6.8 below) by borrowing some ideas from the proofs of [10, Theorem C] and [18, Theorem 2.12]. Later, in Subsection 6.3, via introducing the adapted atomic Musielak-Orlicz Hardy spaces $H_{\mathrm{at}, \mathrm{A}}^{\varphi, q}(\mathcal{X})$ and proving their equivalences to $H_{\mathrm{at}}^{\varphi, q}(\mathcal{X})$ (see Theorem 6.11 below), and some technical lemmas (see Lemmas 4.9 and 6.13 below), we establish the Lusin area function characterizations 
of $H^{\varphi}(\mathcal{X})$ (see Theorem 6.15 below), where the distribution $\left(\mathcal{G}_{0}^{\epsilon}(\varrho, \vartheta)\right)^{\prime}$ is replaced by its homogeneous version $\left(\dot{\mathcal{G}}_{0}^{\epsilon}(\varrho, \vartheta)\right)^{\prime}$ (see Proposition 6.12 below). Using the main results in the above subsections, in Subsection 6.4, we establish the Littlewood-Paley function characterizations of $H^{\varphi}(\mathcal{X})$ (see Theorem 6.16 below).

In Section 7, we give a finite atomic characterization of $H^{\varphi}(\mathcal{X})$ and an improved version in case $q<\infty$. Indeed, in Subsection 7.1, combining some ideas used in the proofs of [35, Theorem 3.4] and [27, Theorem 7.1], under the uniformly dominated convergence condition, we show that $\|\cdot\|_{H_{\text {fin }}^{\varphi, q}(\mathcal{X})}$, with $q \in(q(\varphi), \infty)$, and $\|\cdot\|_{H^{*, \varphi}(\mathcal{X})}$ are equivalent quasi-norms on $H_{\mathrm{fin}}^{\varphi, q}(\mathcal{X}),\|\cdot\|_{H_{\mathrm{fin}}^{\varphi, \infty}(\mathcal{X})}$ and $\|\cdot\|_{H_{\mathrm{at}}^{\varphi, \infty}(\mathcal{X})}$ are equivalent quasi-norms on $H_{\text {fin }}^{\varphi, \infty}(\mathcal{X}) \cap \mathrm{UC}(\mathcal{X})$, and $H_{\text {fin }}^{\varphi, \infty}(\mathcal{X}) \cap \mathrm{UC}(\mathcal{X})$ is dense in $H_{\mathrm{at}}^{\varphi, \infty}(\mathcal{X})$, where $\operatorname{UC}(\mathcal{X})$ denotes the set of uniformly continuous functions on $\mathcal{X}$. The application of the above finite atomic characterizations of $H^{\varphi}(\mathcal{X})$ to the criterion for the boundedness of sublinear operators from $H^{\varphi}(\mathcal{X})$ to a quasi-Banach space is also included in this section. By using some ideas from [7, 45], in Subsection 7.2 for the case $q<\infty$, we remove the uniformly dominated convergence condition of the main results of Subsection 7.1, which essentially improves the corresponding results.

In Section 8, with the help of the finite atomic characterization of $H^{\varphi}(\mathcal{X})$ in Section 7 and some ideas used in the proof of the corresponding Euclidean case (see, for instance, [35, Theorem 3.2]), we find that the dual space of $H^{\varphi}(\mathcal{X})$ is just the Musielak-Orlicz BMO space $\mathrm{BMO}^{\varphi}(\mathcal{X})$ (see Theorem 10.6 below).

In Section 9, applying the criteria of the boundedness of sublinear operators from $H^{\varphi}(\mathcal{X})$ to a quasi-Banach space established in Section 7 , we show the boundedness of Calderón-Zygmund operators on $H^{\varphi}(\mathcal{X})$ (see Theorem 9.2 below).

In Section 10, via the characterizations of $\operatorname{PWM}(\operatorname{BMO}(\mathcal{X}))$ [namely, the set of pointwise multipliers for $\operatorname{BMO}(\mathcal{X})]$ from [52, Example 2.8] in terms of some typical Musielak-Orlicz BMO space $\mathrm{BMO}^{\log }(\mathcal{X})$, we establish another characterization of $\operatorname{PWM}(\operatorname{BMO}(\mathcal{X}))$ (see Theorem 10.6 below) by the typical Musielak-Orlicz Hardy space $H^{\log }(\mathcal{X})$, which is a generalization of [35, Theorem 3.3]. The main difficulty is to find a proper equivalent norm of functions in $\mathrm{BMO}^{\log }(\mathcal{X})$ (see Proposition 10.4 below). We point out that the Ahlfors $n$-regular condition [see (10.1) below] plays important roles in the above characterization of $\operatorname{PWM}(\operatorname{BMO}(\mathcal{X}))$. It is unclear whether or not the Ahlfors $n$-regular assumption can be relaxed.

Finally, we list some conventions on notation. Throughout this article, let $\mathbf{N}:=\{1,2, \ldots\}, \mathbf{Z}_{+}:=\{0\} \cup \mathbf{N}, C$ and $c$ stand for positive constants which are independent of the main parameters, but they may change from line to line. Moreover, we use $C_{(\rho, \alpha, \ldots)}$ and $c_{(\rho, \alpha, \ldots)}$ to denote positive constants depending on the indicated parameters $\rho, \alpha, \ldots$. If, for any real functions $f$ and $g, f \leq C g$, we then write $f \lesssim g$ and, if $f \lesssim g \lesssim f$, we then write $f \sim g$. We also use the following convention: If $f \leq C g$ and $g=h$ or $g \leq h$, we then write $f \lesssim g \sim h$ or $f \lesssim g \lesssim h$, rather than $f \lesssim g=h$ or $f \lesssim g \leq h$. For any subset $E$ of $\mathcal{X}$, we let $\mathbf{1}_{E}$ denote its characteristic function and let $E^{\complement}$ denote its complementary set $\mathcal{X} \backslash E$. For any ball $B$, let $x_{B}$ and $r_{B}$ denote, respectively, the center and the radius of $B$. Furthermore, for any $x, y \in \mathcal{X}, r, \rho \in(0, \infty)$ and ball $B:=B(x, r)$, let

$$
\rho B:=B(x, \rho r), \quad V(x, r):=\mu(B(x, r))=: V_{r}(x) \quad \text { and } \quad V(x, y):=\mu(B(x, d(x, y))) .
$$

For any $p \in[1, \infty]$, we use $p^{\prime}$ to denote its conjugate index, namely, $1 / p+1 / p^{\prime}=1$. 


\section{Preliminaries}

In this section, we recall some basic notions, notation and known results used later in this article.

The following version of the space of test functions on $\mathcal{X}$ was originally introduced by Han et al. [25, Definition 2.2] (see also [26, Definition 2.8]).

Definition 2.1. Let $x_{1} \in \mathcal{X}, r \in(0, \infty), \varrho \in(0,1]$ and $\vartheta \in(0, \infty)$. The space of test functions, $\mathcal{G}\left(x_{1}, r, \varrho, \vartheta\right)$, is defined to be the set of all measurable functions $f$ on $\mathcal{X}$ satisfying that there exists a non-negative constant $C$ such that

(T1) for any $x \in \mathcal{X},|f(x)| \leq C \frac{1}{V_{r}\left(x_{1}\right)+V\left(x_{1}, x\right)}\left[\frac{r}{r+d\left(x_{1}, x\right)}\right]^{\vartheta}$;

(T2) for any $x, y \in \mathcal{X}$ satisfying $d(x, y) \leq\left[r+d\left(x_{1}, x\right)\right] /\left(2 A_{0}\right)$,

$$
|f(x)-f(y)| \leq C\left[\frac{d(x, y)}{r+d\left(x_{1}, x\right)}\right]^{\varrho} \frac{1}{V_{r}\left(x_{1}\right)+V\left(x_{1}, x\right)}\left[\frac{r}{r+d\left(x_{1}, x\right)}\right]^{\vartheta},
$$

where, for any $x, y \in \mathcal{X}$ and $r \in(0, \infty), V_{r}(x):=\mu(B(x, r))$ and $V(x, y):=$ $\mu(B(x, d(x, y)))$. Moreover, for any $f \in \mathcal{G}\left(x_{1}, r, \varrho, \vartheta\right)$, define

$$
\|f\|_{\mathcal{G}\left(x_{1}, r, \varrho, \vartheta\right)}:=\inf \{C: C \text { satisfies }(\mathrm{T} 1) \text { and }(\mathrm{T} 2)\} \text {. }
$$

Fix $x_{1} \in \mathcal{X}$. It is obvious that $\mathcal{G}\left(x_{1}, 1, \varrho, \vartheta\right)$ is a Banach space. For any $x \in \mathcal{X}$ and $r \in(0, \infty)$, it is easy to see that $\mathcal{G}(x, r, \varrho, \vartheta)=\mathcal{G}\left(x_{1}, 1, \varrho, \vartheta\right)$ with equivalent norms. For notational simplicity, we denote $\mathcal{G}\left(x_{1}, 1, \varrho, \vartheta\right)$ simply by $\mathcal{G}(\varrho, \vartheta)$. For any given $\varepsilon \in(0,1]$, let $\mathcal{G}_{0}^{\varepsilon}(\varrho, \vartheta)$ be the completion of the space $\mathcal{G}(\varepsilon, \varepsilon)$ in $\mathcal{G}(\varrho, \vartheta)$ when $\varrho, \vartheta \in(0, \varepsilon]$. Moreover, if $f \in \mathcal{G}_{0}^{\varepsilon}(\varrho, \vartheta)$, we then let $\|f\|_{\mathcal{G}_{0}^{\varepsilon}(\varrho, \vartheta)}:=\|f\|_{\mathcal{G}(\varrho, \vartheta)}$. Also, we let $\stackrel{\mathcal{G}}{0}_{0}^{\varepsilon}(\varrho, \vartheta):=\left\{f \in \mathcal{G}_{0}^{\varepsilon}(\varrho, \vartheta): \int_{\mathcal{X}} f(x) d \mu(x)=0\right\}$.

The dual spaces $\left(\mathcal{G}_{0}^{\varepsilon}(\varrho, \vartheta)\right)^{\prime}$ and $\left(\stackrel{\mathcal{G}}{0}_{0}^{\varepsilon}(\varrho, \vartheta)\right)^{\prime}$ are defined, respectively, to be the sets of all continuous linear functionals $\mathcal{L}$ from $\mathcal{G}_{0}^{\varepsilon}(\varrho, \vartheta)$ to $\mathbf{C}$ and from $\dot{\mathcal{G}}_{0}^{\varepsilon}(\varrho, \vartheta)$ to $\mathbf{C}$, and both are equipped with the weak-* topology. For any $f \in\left(\mathcal{G}_{0}^{\varepsilon}(\varrho, \vartheta)\right)^{\prime}$ and $\phi \in \mathcal{G}_{0}^{\varepsilon}(\varrho, \vartheta)$, we use the pairing $\langle f, \phi\rangle$ to denote the action of $f$ on $\phi$. In particular, when $f$ is locally integrable, it makes sense to write

$$
\langle f, \phi\rangle=\int_{\mathcal{X}} f(x) \phi(x) d \mu(x) .
$$

Then we recall the following notion of Orlicz functions. A function $\phi:[0, \infty) \rightarrow$ $[0, \infty)$ is called an Orlicz function if it is non-decreasing and $\phi(0)=0, \phi(t)>0$ if $t \in(0, \infty)$, and $\lim _{t \rightarrow \infty} \phi(t)=\infty$.

Now, we recall the notions of uniformly lower (resp., upper) types on MusielakOrlicz functions from [31].

Definition 2.2. For a given function $\varphi: \mathcal{X} \times[0, \infty) \rightarrow[0, \infty)$ such that, for almost every $x \in \mathcal{X}, \varphi(x, \cdot)$ is an Orlicz function, $\varphi$ is said to be of uniformly lower (resp., upper) type $p$ if there exists a positive constant $C_{(p)}$, depending on $p$, such that, for almost every $x \in \mathcal{X}, t \in[0, \infty)$ and $s \in(0,1)$ [resp., $s \in[1, \infty)]$,

$$
\varphi(x, s t) \leq C_{(p)} s^{p} \varphi(x, t) .
$$

The function $\varphi$ is said to be of uniformly lower (resp., upper) type if it is of uniformly lower (resp., upper) type $p$ for some $p \in(0, \infty)$ and let

$$
i(\varphi):=\sup \{p \in(0, \infty): \varphi \text { is of uniformly lower type } p\} .
$$

Now, we recall the following notion of the set of Muckenhoupt weights on $\mathcal{X}$ from [58]. 
Definition 2.3. A measurable function $w: \mathcal{X} \rightarrow(0, \infty)$ is said to belong to the set of Muckenhoupt weights for some $q \in[1, \infty)$, denoted by $\varphi \in A_{q}(\mathcal{X})$, if, when $q \in(1, \infty)$,

or

$$
[w]_{A_{q}(\mathcal{X})}:=\sup _{B \subset \mathcal{X}} \frac{1}{[\mu(B)]^{q}} \int_{B} w(x) d \mu(x)\left\{\int_{B}[w(y)]^{-1 /(q-1)} d \mu(y)\right\}^{q-1}<\infty
$$

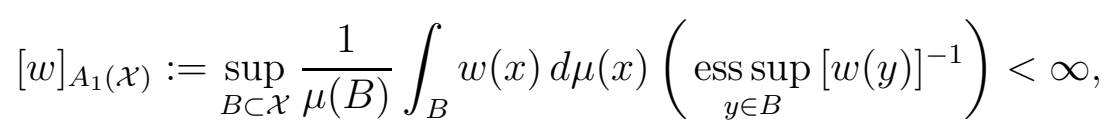

where the suprema are taken over all balls $B \subset \mathcal{X}$. Let

$$
A_{\infty}(\mathcal{X}):=\bigcup_{q \in[1, \infty)} A_{q}(\mathcal{X})
$$

We recall the notions of the uniformly Muckenhoupt condition and the uniformly reverse Hölder condition from [31].

Definition 2.4. A function $\varphi: \mathcal{X} \times[0, \infty) \rightarrow[0, \infty)$ is said to satisfy the uniformly Muckenhoupt condition for some $q \in[1, \infty)$, denoted by $\varphi \in \mathbf{A}_{q}(\mathcal{X})$, if, when $q \in(1, \infty)$,

$$
[\varphi]_{\mathbf{A}_{q}(\mathcal{X})}:=\sup _{t \in(0, \infty)} \sup _{B \subset \mathcal{X}} \frac{1}{[\mu(B)]^{q}} \int_{B} \varphi(x, t) d \mu(x)\left\{\int_{B}[\varphi(y, t)]^{-1 /(q-1)} d \mu(y)\right\}^{q-1}<\infty
$$

or

$$
[\varphi]_{\mathbf{A}_{1}(\mathcal{X})}:=\sup _{t \in(0, \infty)} \sup _{B \subset \mathcal{X}} \frac{1}{\mu(B)} \int_{B} \varphi(x, t) d \mu(x)\left(\underset{y \in B}{\operatorname{ess} \sup }[\varphi(y, t)]^{-1}\right)<\infty,
$$

where the first suprema are taken over all $t \in(0, \infty)$ and the second ones over all balls $B \subset \mathcal{X}$.

Let

$$
\mathbf{A}_{\infty}(\mathcal{X}):=\bigcup_{q \in[1, \infty)} \mathbf{A}_{q}(\mathcal{X})
$$

A function $\varphi: \mathcal{X} \times[0, \infty) \rightarrow[0, \infty)$ is said to satisfy the uniformly reverse Hölder condition for some $q \in(1, \infty]$, denoted by $\varphi \in \mathbf{R H}_{q}(\mathcal{X})$, if, when $q \in(1, \infty)$,

$$
[\varphi]_{\mathbf{R H}_{q}(\mathcal{X})}:=\sup _{t \in(0, \infty)} \sup _{B \subset \mathcal{X}}\left\{\frac{1}{\mu(B)} \int_{B}[\varphi(x, t)]^{q} d \mu(x)\right\}^{1 / q}\left\{\frac{1}{\mu(B)} \int_{B} \varphi(y, t) d \mu(y)\right\}^{-1}<\infty
$$

or

$$
[\varphi]_{\mathbf{R} \mathbf{H}_{\infty}(\mathcal{X})}:=\sup _{t \in(0, \infty)} \sup _{B \subset \mathcal{X}}\left\{\operatorname{esssup}_{y \in B} \varphi(y, t)\right\}\left\{\frac{1}{\mu(B)} \int_{B} \varphi(y, t) d \mu(y)\right\}^{-1}<\infty
$$

where the first suprema are taken over all $t \in(0, \infty)$ and the second ones over all balls $B \subset \mathcal{X}$.

The following notion of growth functions on spaces of homogeneous type were taken from [31].

Definition 2.5. A function $\varphi: \mathcal{X} \times[0, \infty) \rightarrow[0, \infty)$ is called a growth function if the following conditions are satisfied:

(i) $\varphi$ is a Musielak-Orlicz function, namely,

$(\mathrm{i})_{1}$ the function $\varphi(x, \cdot):[0, \infty) \rightarrow[0, \infty)$ is an Orlicz function for almost every $x \in \mathcal{X}$; 
(i) $)_{2}$ the function $\varphi(\cdot, t)$ is measurable for any $t \in[0, \infty)$.

(ii) $\varphi \in \mathbf{A}_{\infty}(\mathcal{X})$.

(iii) $\varphi$ is of uniformly lower type $p$ for some $p \in(0,1]$ and of uniformly upper type 1.

In what follows, let $M$ denote the Hardy-Littlewood maximal operator defined by setting, for any $f \in L_{\text {loc }}^{1}(\mathcal{X})$ (the space of all locally integrable functions on $\mathcal{X}$ ) and $x \in \mathcal{X}$,

$$
M(f)(x):=\sup _{B \ni x} \frac{1}{\mu(B)} \int_{B}|f(y)| d \mu(y),
$$

where the supremum is taken over all balls $B$ of $\mathcal{X}$ containing $x$.

Now, we list some basic properties of $\mathbf{A}_{q}(\mathcal{X})$ for any $q \in[1, \infty)$ whose proofs are similar to those of the Euclidean case in [59, Lemma 1.1.3]; the details are omitted here (see [31, Lemmas 2.11 and 2.12] for some details).

Lemma 2.6. The following conclusions hold true.

(i) $\mathbf{A}_{1}(\mathcal{X}) \subset \mathbf{A}_{p}(\mathcal{X}) \subset \mathbf{A}_{q}(\mathcal{X})$ for any $p, q$ satisfying $1 \leq p \leq q<\infty$.

(ii) $\mathbf{R H}_{\infty}(\mathcal{X}) \subset \mathbf{R} \mathbf{H}_{q}(\mathcal{X}) \subset \mathbf{R} \mathbf{H}_{p}(\mathcal{X})$ for any $p$, $q$ satisfying $1<p \leq q \leq \infty$.

(iii) If $q \in[1, \infty)$ and $\varphi \in \mathbf{A}_{q}(\mathcal{X})$, then there exists a positive constant $C$ such that, for any ball $B \subset \mathcal{X}$, measurable function $f$ and $t \in(0, \infty)$,

$$
\left[\frac{1}{\mu(B)} \int_{B}|f(x)| d \mu(x)\right]^{q} \leq C \frac{1}{\varphi(B, t)} \int_{B}|f(x)|^{q} \varphi(x, t) d \mu(x),
$$

here and hereafter, for any measurable set $E \subset \mathcal{X}$ and $t \in[0, \infty)$, let

$$
\varphi(E, t):=\int_{E} \varphi(x, t) d \mu(x) .
$$

(iv) If $\varphi \in \mathbf{A}_{q}(\mathcal{X})$ with $q \in[1, \infty)$, then there exists a positive constant $C$ such that, for any ball $B \subset \mathcal{X}$, measurable set $E \subset B$ and $t \in(0, \infty)$,

$$
\frac{\varphi(B, t)}{\varphi(E, t)} \leq C\left[\frac{\mu(B)}{\mu(E)}\right]^{q} .
$$

(v) If $q \in(1, \infty)$ and $\varphi \in \mathbf{A}_{q}(\mathcal{X})$, then there exists a positive constant $C$ such that, for any $f \in L_{\mathrm{loc}}^{1}(\mathcal{X})$ and $t \in[0, \infty)$,

$$
\int_{\mathcal{X}}[M(f)(x)]^{q} \varphi(x, t) d \mu(x) \leq C \int_{\mathcal{X}}|f(x)|^{q} \varphi(x, t) d \mu(x) .
$$

We then recall the definition of Musielak-Orlicz spaces from [31, Definition 2.8].

Definition 2.7. Let $\varphi$ be a Musielak-Orlicz function. The Musielak-Orlicz space $L^{\varphi}(\mathcal{X})$ is defined to be the set of all measurable functions $f$ such that, for some $\lambda \in(0, \infty)$,

$$
\int_{\mathcal{X}} \varphi(x,|f(x)| / \lambda) d \mu(x)<\infty
$$

equipped with the Luxembourg (also called the Luxembourg-Nakano) (quasi-)norm

$$
\|f\|_{L^{\varphi}(\mathcal{X})}:=\inf \left\{\lambda \in(0, \infty): \int_{\mathcal{X}} \varphi(x,|f(x)| / \lambda) d \mu(x) \leq 1\right\} .
$$

We further recall some basic properties of $L^{\varphi}(\mathcal{X})$, which are easy generalizations of the corresponding properties of the Euclidean case in [59, Lemmas 1.1.6 and 1.1.10] to any space of homogeneous type; the details are omitted. 
Real-variable characterizations of Musielak-Orlicz Hardy spaces on spaces of homogeneous type 351

Lemma 2.8. Let $\varphi$ be a growth function as in Definition 2.5. Then the following conclusions hold true.

(i) The growth function $\varphi$ is uniformly $\sigma$-quasi-subadditive on $\mathcal{X} \times[0, \infty)$, namely, there exists a positive constant $C$ such that, for any $\left(x, t_{j}\right) \in \mathcal{X} \times[0, \infty)$ with $j \in \mathbf{N}$,

$$
\varphi\left(x, \sum_{j=1}^{\infty} t_{j}\right) \leq C \sum_{j=1}^{\infty} \varphi\left(x, t_{j}\right) .
$$

(ii) For any $f \in L^{\varphi}(\mathcal{X}) \backslash\{0\}$,

$$
\int_{\mathcal{X}} \varphi\left(x, \frac{|f(x)|}{\|f\|_{L^{\varphi}(\mathcal{X})}}\right) d \mu(x)=1 .
$$

(iii) For any $\left\{f_{k}\right\}_{k \in \mathbf{N}} \subset L^{\varphi}(\mathcal{X}), \lim _{k \rightarrow \infty}\left\|f_{k}\right\|_{L^{\varphi}(\mathcal{X})}=0$ if and only if

$$
\lim _{k \rightarrow \infty} \int_{\mathcal{X}} \varphi\left(x,\left|f_{k}(x)\right|\right) d \mu(x)=0 .
$$

(iii) For any $(x, t) \in \mathcal{X} \times[0, \infty), \widetilde{\varphi}(x, t):=\int_{0}^{t} \frac{\varphi(x, s)}{s} d s$ is a growth function, which is equivalent to $\varphi$; moreover, for almost every $x \in \mathcal{X}, \widetilde{\varphi}(x, \cdot)$ is continuous and strictly increasing.

In what follows, for any $f \in L_{\text {loc }}^{1}(\mathcal{X})$ and any ball $B \subset \mathcal{X}$, let

$$
m_{B}(f):=\frac{1}{\mu(B)} \int_{B} f(x) d \mu(x) .
$$

Then we generalize the notion of Musielak-Orlicz BMO spaces $\operatorname{BMO}^{\varphi}\left(\mathbf{R}^{n}\right)$ in [35] to any space of homogeneous type as follows.

Definition 2.9. A function $f \in L_{\text {loc }}^{1}(\mathcal{X})$ is said to belong to $\operatorname{BMO}^{\varphi}(\mathcal{X})$ if

$$
\|f\|_{\mathrm{BMO}^{\varphi}(\mathcal{X})}:=\sup _{B} \frac{1}{\left\|\mathbf{1}_{B}\right\|_{L^{\varphi}(\mathcal{X})}} \int_{B}\left|f(x)-m_{B}(f)\right| d \mu(x)<\infty,
$$

where the supremum is taken over all balls $B \subset \mathcal{X}$.

Remark 2.10. Fix $x_{0} \in \mathcal{X}$. A typical example of the space $\operatorname{BMO}^{\varphi}(\mathcal{X})$ is the space $\mathrm{BMO}^{\log }(\mathcal{X})$ which is just the space $\mathrm{BMO}^{\varphi}(\mathcal{X})$ with

$$
\varphi(x, t):=\frac{t}{\log \left(e+d\left(x, x_{0}\right)\right)+\log (e+t)}, \quad \forall x \in \mathcal{X}, \forall t \in[0, \infty) .
$$

We further introduce the following notion of Musielak-Orlicz Campanato spaces $\operatorname{Lip}_{\varphi, q}(\mathcal{X})$, which is a generalization of the corresponding Euclidean case in [43] to any space of homogeneous type.

Definition 2.11. A function $f \in L_{\text {loc }}^{1}(\mathcal{X})$ is said to belong to the MusielakOrlicz Campanato space $\operatorname{Lip}_{\varphi, q}(\mathcal{X})$ if

$$
\begin{aligned}
\|f\|_{\operatorname{Lip}_{\varphi, q}(\mathcal{X})} & :=\sup _{B} \frac{1}{\left\|\mathbf{1}_{B}\right\|_{L^{\varphi}(\mathcal{X})}}\left\{\int_{B}\left[\frac{\left|f(x)-m_{B}(f)\right|}{\varphi\left(x,\left\|\mathbf{1}_{B}\right\|_{L^{\varphi}(\mathcal{X})}^{-1}\right)}\right]^{q} \varphi\left(x,\left\|\mathbf{1}_{B}\right\|_{L^{\varphi}(\mathcal{X})}^{-1}\right) d \mu(x)\right\}^{1 / q} \\
& <\infty
\end{aligned}
$$

where the supremum is taken over all balls $B \subset \mathcal{X}$.

Remark 2.12. Obviously, $\operatorname{Lip}_{\varphi, 1}(\mathcal{X})=\operatorname{BMO}^{\varphi}(\mathcal{X})$. 


\section{John-Nirenberg inequality of $\mathrm{BMO}^{\varphi}(\mathcal{X})$}

In this section, we obtain the John-Nirenberg inequality and establish several equivalent characterizations of $\mathrm{BMO}^{\varphi}(\mathcal{X})$. The following John-Nirenberg inequality for $\mathrm{BMO}^{\varphi}(\mathcal{X})$ is similar to the corresponding Euclidean case in [43, Theorem 2.5]. For the convenience of the reader, we present some details here.

Theorem 3.1. Let $\varphi$ be a growth function as in Definition 2.5 and $f \in \mathrm{BMO}^{\varphi}(\mathcal{X})$. Then there exist positive constants $C_{1}, C_{2}$ and $C_{3}$, independent of $f$, such that, for any ball $B \subset \mathcal{X}$ and $t \in(0, \infty)$, if $\varphi \in \mathbf{A}_{1}(\mathcal{X})$, then

$$
\varphi\left(\left\{x \in B: \frac{\left|f(x)-m_{B}(f)\right|}{\varphi\left(x,\left\|\mathbf{1}_{B}\right\|_{L^{\varphi}(\mathcal{X})}^{-1}\right)}>t\right\},\left\|\mathbf{1}_{B}\right\|_{L^{\varphi}(\mathcal{X})}^{-1}\right) \leq C_{1} \exp \left\{-\frac{C_{2} t}{\|f\|_{\operatorname{Lip}_{\varphi, 1}(\mathcal{X})}\left\|\mathbf{1}_{B}\right\|_{L^{\varphi}(\mathcal{X})}}\right\}
$$

and, if $\varphi \in \mathbf{A}_{q}(\mathcal{X})$ for some $q \in(1, \infty)$, then

$$
\varphi\left(\left\{x \in B: \frac{\left|f(x)-m_{B}(f)\right|}{\varphi\left(x,\left\|\mathbf{1}_{B}\right\|_{L^{\varphi}(\mathcal{X})}^{-1}\right)}>t\right\},\left\|\mathbf{1}_{B}\right\|_{L^{\varphi}(\mathcal{X})}^{-1}\right) \leq C_{3}\left[1+\frac{t}{\|f\|_{\operatorname{Lip}_{\varphi, 1}(\mathcal{X})}\left\|\mathbf{1}_{B}\right\|_{L^{\varphi}(\mathcal{X})}}\right]^{-q^{\prime}}
$$

where $m_{B}(f)$ is as in $(2.3)$ and $q^{\prime}:=q /(q-1)$.

To prove Theorem 3.1, we need the following two technical lemmas. The first lemma is an easy generalization of the corresponding Euclidean case in [51, Lemma (3.1)] to any space of homogeneous type; the details are omitted.

Lemma 3.2. Let $q \in(1, \infty)$ and $\frac{1}{q}+\frac{1}{q^{\prime}}=1$. If $w \in A_{q}(\mathcal{X})$, then there exists a positive constant $C$ such that, for any ball $B \subset \mathcal{X}$ and $t \in(0, \infty)$,

$$
w(\{x \in B: w(x)<t\}) \leq C\left[t \frac{\mu(B)}{w(B)}\right]^{q^{\prime}} w(B),
$$

here and hereafter, for any non-negative measurable function $w$ and measurable set $E \subset \mathcal{X}$

$$
w(E):=\int_{E} w(x) d \mu(x)
$$

The second lemma is a generalization of the weighted version of CalderónZygmund decompositions on the Euclidean case in [51, Lemma (3.2)] to any space of homogeneous type.

Lemma 3.3. Let $w: \mathcal{X} \rightarrow(0, \infty)$ be a measurable function satisfying the doubling condition, namely, there exists a positive constant $\widetilde{C}_{0}$ such that, for any ball $B \subset \mathcal{X}, w(2 B) \leq \widetilde{C}_{0} w(B)$ and, for a given ball $B_{0} \subset \mathcal{X}$ and $\sigma \in(0, \infty)$, let $f$ be a non-negative function which satisfies that

$$
\frac{1}{w\left(B_{0}\right)} \int_{B_{0}} f(x) w(x) d \mu(x) \leq \sigma .
$$

Then there exists a sequence $\left\{B_{k}\right\}_{k \in I}$ ( $I$ is some countable index set) of disjoint balls such that, for almost every $x \in B_{0} \backslash \bigcup_{k \in I}\left(4 A_{0} B_{k}\right)$ with $A_{0}$ same as in (1.2),

$$
f(x) \leq \sigma
$$

and, for any $k \in I$,

$$
\sigma<\frac{1}{w\left(B_{k}\right)} \int_{B_{k} \cap B_{0}} f(x) w(x) d \mu(x) \leq \widetilde{C}_{0} \sigma
$$


Proof. For a fixed ball $B_{0}$ and $\sigma \in(0, \infty)$, let $E:=\left\{x \in \mathcal{X}: f(x) \mathbf{1}_{B_{0}}(x)>\sigma\right\} \subset$ $B_{0}$. Observe that, by the generalized Lebesgue differential theorem (see, for instance, [29, Theorem 1.8]), we know that, for almost every $x \in E$, there exists a ball $B_{x}^{\prime} \subset \mathcal{X}$ such that

$$
\frac{1}{w\left(B_{x}^{\prime}\right)} \int_{B_{x}^{\prime}} f(y) \mathbf{1}_{B_{0}}(y) w(y) d \mu(y)>\sigma .
$$

Let $B_{x}$ be the largest ball, satisfying (3.2), concentric with $B_{x}^{\prime}$ and having the radius of the form $2^{m} r_{B_{x}^{\prime}}$ with $m \in \mathbf{Z}_{+}$. Then, for $\widetilde{B}_{x}:=2 B_{x}$,

$$
\frac{1}{w\left(\widetilde{B}_{x}\right)} \int_{\widetilde{B}_{x}} f(y) \mathbf{1}_{B_{0}}(y) w(y) d \mu(y) \leq \sigma .
$$

From the Vitali-Wiener type covering lemma (see, for instance, [10, Theorem (3.1)]), it follows that there exists a disjoint subsequence $\left\{B_{k}\right\}_{k \in I}$ ( $I$ is some countable index set) of $\left\{B_{x}\right\}_{x \in E}$ such that $E \subset \bigcup_{k \in I}\left(4 A_{0} B_{k}\right)$. Thus, (3.1) follows immediately.

Moreover, by the doubling condition of $w$, we further have

$$
\sigma<\frac{1}{w\left(B_{k}\right)} \int_{B_{k} \cap B_{0}} f(y) w(y) d \mu(y) \leq \frac{w\left(\widetilde{B}_{k}\right)}{w\left(B_{k}\right)} \frac{1}{w\left(\widetilde{B}_{k}\right)} \int_{\widetilde{B}_{k}} f(y) \mathbf{1}_{B_{0}}(y) w(y) d \mu(y) \leq \widetilde{C}_{0} \sigma
$$

which completes the proof of Lemma 3.3.

Now, we are ready to prove Theorem 3.1.

Proof of Theorem 3.1. Let $f \in \operatorname{Lip}_{\varphi, 1}(\mathcal{X})$ and fix any ball $B_{0} \subset \mathcal{X}$. By the homogeneity of the norm of $f \in \operatorname{Lip}_{\varphi, 1}(\mathcal{X})$, without loss of generality, we may assume that $\|f\|_{\operatorname{Lip}_{\varphi, 1}(\mathcal{X})}=\left\|\mathbf{1}_{B_{0}}\right\|_{L^{\varphi}(\mathcal{X})}^{-1} ;$ otherwise, we may replace $f$ by

$$
\frac{f}{\|f\|_{\operatorname{Lip}_{\varphi, 1}(\mathcal{X})}\left\|\mathbf{1}_{B_{0}}\right\|_{L^{\varphi}(\mathcal{X})}} \text {. }
$$

For any $t \in(0, \infty)$ and ball $B \subset B_{0}$, let

$$
\lambda(t, B):=\varphi\left(\left\{x \in B: \frac{\left|f(x)-m_{B}(f)\right|}{\varphi\left(x,\left\|\mathbf{1}_{B_{0}}\right\|_{L^{\varphi}(\mathcal{X})}^{-1}\right)}>t\right\},\left\|\mathbf{1}_{B_{0}}\right\|_{L^{\varphi}(\mathcal{X})}^{-1}\right)
$$

and

$$
\mathcal{F}(t):=\sup _{B \subset B_{0}} \frac{\lambda(t, B)}{\varphi\left(B,\left\|\mathbf{1}_{B_{0}}\right\|_{L^{\varphi}(\mathcal{X})}^{-1}\right)} .
$$

From $\lambda(t, B) \leq \varphi\left(B,\left\|\mathbf{1}_{B_{0}}\right\|_{L^{\varphi}(\mathcal{X})}^{-1}\right)$, it follows that, for any $t \in(0, \infty), \mathcal{F}(t) \leq 1$. By $\|f\|_{\operatorname{Lip}_{\varphi, 1}(\mathcal{X})}=\left\|\mathbf{1}_{B_{0}}\right\|_{L^{\varphi}(\mathcal{X})}^{-1}$ and the uniformly upper type 1 property of $\varphi$, we obtain

$$
\begin{aligned}
& \frac{1}{\varphi\left(B,\left\|\mathbf{1}_{B_{0}}\right\|_{L^{\varphi}(\mathcal{X})}^{-1}\right)} \int_{B}\left|f(x)-m_{B}(f)\right| d \mu(x) \\
& \leq \frac{\left\|\mathbf{1}_{B}\right\|_{L^{\varphi}(\mathcal{X})}}{\varphi\left(B,\left\|\mathbf{1}_{B_{0}}\right\|_{L^{\varphi}(\mathcal{X})}^{-1}\right)\left\|\mathbf{1}_{B_{0}}\right\|_{L^{\varphi}(\mathcal{X})}} \leq \frac{\widetilde{C}_{1}\left\|\mathbf{1}_{B}\right\|_{L^{\varphi}(\mathcal{X})}}{\varphi\left(B,\left\|\mathbf{1}_{B}\right\|_{L^{\varphi}(\mathcal{X})}^{-1}\right)\left\|\mathbf{1}_{B}\right\|_{L^{\varphi}(\mathcal{X})}}=\widetilde{C}_{1}
\end{aligned}
$$

for the positive constant $\widetilde{C}_{1}:=\frac{1}{C_{(1)}}$ with $C_{(1)}$ as in Definition 2.2.

Furthermore, let $\sigma \in\left[\widetilde{C}_{1}, \infty\right)$. From Lemma 3.3 with $f(\cdot)$ and $w(\cdot)$ replaced, respectively, by

$$
\left[\varphi\left(\cdot,\left\|\mathbf{1}_{B_{0}}\right\|_{L^{\varphi}(\mathcal{X})}^{-1}\right)\right]^{-1}\left|f(\cdot)-m_{B}(f)\right|
$$


and $\varphi\left(\cdot,\left\|\mathbf{1}_{B_{0}}\right\|_{L^{\varphi}(\mathcal{X})}^{-1}\right)$, we deduce that there exist a disjoint sequence $\left\{B_{k}\right\}_{k \in I}$, with $I$ as in Lemma 3.3, of balls and a positive constant $\widetilde{C}_{0}$ as in Lemma 3.3 such that, for almost every $x \in B \backslash \bigcup_{k \in I}\left(4 A_{0} B_{k}\right)$,

$$
\left[\varphi\left(x,\left\|\mathbf{1}_{B_{0}}\right\|_{L^{\varphi}(\mathcal{X})}^{-1}\right)\right]^{-1}\left|f(x)-m_{B}(f)\right| \leq \sigma
$$

and, for any $k \in I$,

$$
\sigma<\frac{1}{\varphi\left(B_{k},\left\|\mathbf{1}_{B_{0}}\right\|_{L^{\varphi}(\mathcal{X})}^{-1}\right)} \int_{B_{k} \cap B}\left|f(x)-m_{B}(f)\right| d \mu(x) \leq \widetilde{C}_{0} \sigma .
$$

Thus, by this and (3.4), we have

$$
\begin{aligned}
& \sum_{k \in I} \varphi\left(B_{k},\left\|\mathbf{1}_{B_{0}}\right\|_{L^{\varphi}(\mathcal{X})}^{-1}\right) \leq \frac{1}{\sigma} \sum_{k \in I} \int_{B_{k} \cap B}\left|f(x)-m_{B}(f)\right| d \mu(x) \\
& \leq \frac{1}{\sigma} \int_{B}\left|f(x)-m_{B}(f)\right| d \mu(x) \leq \frac{\widetilde{C}_{1}}{\sigma} \varphi\left(B,\left\|\mathbf{1}_{B_{0}}\right\|_{L^{\varphi}(\mathcal{X})}^{-1}\right) .
\end{aligned}
$$

If $t \in[\sigma, \infty)$, from (3.5) and Lemma 2.6(iv), we then deduce that

$$
\begin{aligned}
\lambda(t, B) & =\varphi\left(\left\{x \in B: \frac{\left|f(x)-m_{B}(f)\right|}{\varphi\left(x,\left\|\mathbf{1}_{B_{0}}\right\|_{L^{\varphi}(\mathcal{X})}^{-1}\right)}>t\right\},\left\|\mathbf{1}_{B_{0}}\right\|_{L^{\varphi}(\mathcal{X})}^{-1}\right) \\
& \leq \sum_{k \in I} \varphi\left(\left\{x \in 4 A_{0} B_{k}: \frac{\left|f(x)-m_{B}(f)\right|}{\varphi\left(x,\left\|\mathbf{1}_{B_{0}}\right\|_{L^{\varphi}(\mathcal{X})}^{-1}\right)}>t\right\},\left\|\mathbf{1}_{B_{0}}\right\|_{L^{\varphi}(\mathcal{X})}^{-1}\right) \\
& \lesssim \sum_{k \in I} \varphi\left(\left\{x \in B_{k}: \frac{\left|f(x)-m_{B}(f)\right|}{\varphi\left(x,\left\|\mathbf{1}_{B_{0}}\right\|_{L^{\varphi}(\mathcal{X})}^{-1}\right)}>t\right\},\left\|\mathbf{1}_{B_{0}}\right\|_{L^{\varphi}(\mathcal{X})}^{-1}\right) .
\end{aligned}
$$

Thus, for any $t \in[\sigma, \infty)$ and any $s \in[0, t]$, we write

$$
\begin{aligned}
\lambda(t, B) \leq & \sum_{k \in I} \lambda\left(t-s, B_{k}\right) \\
& +\sum_{k \in I} \varphi\left(\left\{x \in B_{k}: \frac{\left|m_{B_{k}}(f)-m_{B}(f)\right|}{\varphi\left(x,\left\|\mathbf{1}_{B_{0}}\right\|_{L^{\varphi}(\mathcal{X})}^{-1}\right)}>s\right\},\left\|\mathbf{1}_{B_{0}}\right\|_{L^{\varphi}(\mathcal{X})}^{-1}\right) \\
= & : \mathrm{I}_{1}+\mathrm{I}_{2} .
\end{aligned}
$$

For $\mathrm{I}_{1}$, by (3.3), (3.7) and some arguments similar to those used in the estimation of $\mathrm{I}_{1}$ in the proof of [43, Theoem 2.5], we then obtain

$$
\mathrm{I}_{1} \leq \frac{\widetilde{C}_{1}}{\sigma} \mathcal{F}(t-s) \varphi\left(B,\left\|\mathbf{1}_{B_{0}}\right\|_{L^{\varphi}(\mathcal{X})}^{-1}\right) .
$$

Moreover, from (3.6), we deduce that

$$
\begin{aligned}
\left|m_{B_{k}}(f)-m_{B}(f)\right| & \leq \frac{1}{\mu\left(B_{k}\right)} \int_{B_{k}}\left|f(x)-m_{B}(f)\right| d \mu(x) \\
& \leq \widetilde{C}_{0} \sigma \frac{\varphi\left(B_{k},\left\|\mathbf{1}_{B_{0}}\right\|_{L^{\varphi}(\mathcal{X})}^{-1}\right)}{\mu\left(B_{k}\right)} .
\end{aligned}
$$

We further consider the following two cases. 
Real-variable characterizations of Musielak-Orlicz Hardy spaces on spaces of homogeneous type 355

Case 1. $\varphi \in \mathbf{A}_{1}(\mathcal{X})$. In this case, we know that there exists a positive constant $\widetilde{C}_{2}$ such that

$$
\frac{\varphi\left(B_{k},\left\|\mathbf{1}_{B_{0}}\right\|_{L^{\varphi}(\mathcal{X})}^{-1}\right)}{\mu\left(B_{k}\right)} \leq \widetilde{C}_{2} \underset{y \in B_{k}}{\operatorname{essinf}} \varphi\left(y,\left\|\mathbf{1}_{B_{0}}\right\|_{L^{\varphi}(\mathcal{X})}^{-1}\right)
$$

which, together with (3.10), implies that

$$
\begin{aligned}
& \varphi\left(\left\{x \in B_{k}: \frac{\left|m_{B_{k}}(f)-m_{B}(f)\right|}{\varphi\left(x,\left\|\mathbf{1}_{B_{0}}\right\|_{L^{\varphi}(\mathcal{X})}^{-1}\right)}>s\right\},\left\|\mathbf{1}_{B_{0}}\right\|_{L^{\varphi}(\mathcal{X})}^{-1}\right) \\
& \leq \varphi\left(\left\{x \in B_{k}: \frac{\widetilde{C}_{0} \widetilde{C}_{2} \sigma \underset{y \in B_{k}}{\operatorname{essinf}} \varphi\left(y,\left\|\mathbf{1}_{B_{0}}\right\|_{L^{\varphi}(\mathcal{X})}^{-1}\right)}{\varphi\left(x,\left\|\mathbf{1}_{B_{0}}\right\|_{L^{\varphi}(\mathcal{X})}^{-1}\right)}>s\right\},\left\|\mathbf{1}_{B_{0}}\right\|_{L^{\varphi}(\mathcal{X})}^{-1}\right) .
\end{aligned}
$$

Now, we choose $\sigma:=2 \widetilde{C}_{1}$ and $s:=2 \widetilde{C}_{0} \widetilde{C}_{1} \widetilde{C}_{2}$. Then we observe that, if $t \in(s, \infty)$, then $\sigma \in\left(\widetilde{C}_{1}, t\right)$ and $s \in(0, t)$ as required.

By (3.8) and (3.11), we conclude that

$$
\mathrm{I}_{2} \leq \sum_{k \in I} \varphi\left(\left\{x \in B_{k}: \frac{\underset{y \in B_{k}}{\operatorname{essinf}} \varphi\left(y,\left\|\mathbf{1}_{B_{0}}\right\|_{L^{\varphi}(\mathcal{X})}^{-1}\right)}{\varphi\left(x,\left\|\mathbf{1}_{B_{0}}\right\|_{L^{\varphi}(\mathcal{X})}^{-1}\right)}>1\right\},\left\|\mathbf{1}_{B_{0}}\right\|_{L^{\varphi}(\mathcal{X})}^{-1}\right)=0
$$

which, combined with (3.8) and (3.9), further implies that, for any $t \in(s, \infty)$ and $B \subset B_{0}$,

$$
\lambda(t, B) \leq \frac{1}{2} \mathcal{F}(t-s) \varphi\left(B,\left\|\mathbf{1}_{B_{0}}\right\|_{L^{\varphi}(\mathcal{X})}^{-1}\right)
$$

Thus, if $t \in(s, \infty)$, then $\mathcal{F}(t) \leq \frac{1}{2} \mathcal{F}(t-s)$.

Moreover, if $m \in \mathbf{N}$ and $t \in(m s,(m-1) s]$, then

$$
\mathcal{F}(t) \leq \frac{1}{2} \mathcal{F}(t-s) \leq \cdots \leq \frac{1}{2^{m}} \mathcal{F}(t-m s)
$$

By $\mathcal{F}(t-m s) \leq 1$ and $m \geq \frac{t}{s}-1$, we conclude that

$$
\mathcal{F}(t) \leq 2^{-m} \leq 2^{1-\frac{t}{s}}=2 e^{-\left(\frac{t}{s} \log 2\right)}
$$

Let $C_{1}:=2$ and $C_{2}:=\frac{1}{s} \log 2$. Then we conclude that, for any given $\varphi \in \mathbf{A}_{1}(\mathcal{X})$ and any $s \in(0, t)$,

$$
\varphi\left(\left\{x \in B: \frac{\left|f(x)-m_{B}(f)\right|}{\varphi\left(x,\left\|\mathbf{1}_{B}\right\|_{L^{\varphi}(\mathcal{X})}^{-1}\right)}>t\right\},\left\|\mathbf{1}_{B}\right\|_{L^{\varphi}(\mathcal{X})}^{-1}\right) \leq 2 e^{-\left(\frac{1}{s} \log 2\right) t}=C_{1} e^{-C_{2} t}
$$

which implies the desired conclusion of Theorem 3.1 in Case 1. 
Case 2. $\varphi \in \mathbf{A}_{q}(\mathcal{X})$ for some $q \in(1, \infty)$. In this case, by (3.8), (3.10), Lemma 3.2 and (3.7), we know that

$$
\begin{aligned}
\mathrm{I}_{2} & \leq \sum_{k \in I} \varphi\left(\left\{x \in B_{k}: \frac{\widetilde{C}_{0} \sigma \varphi\left(B_{k},\left\|\mathbf{1}_{B_{0}}\right\|_{L^{\varphi}(\mathcal{X})}^{-1}\right)}{\mu\left(B_{k}\right) \varphi\left(x,\left\|\mathbf{1}_{B_{0}}\right\|_{L^{\varphi}(\mathcal{X})}^{-1}\right)}>s\right\},\left\|\mathbf{1}_{B_{0}}\right\|_{L^{\varphi}(\mathcal{X})}^{-1}\right) \\
& =\sum_{k \in I} \varphi\left(\left\{x \in B_{k}: \varphi\left(x,\left\|\mathbf{1}_{B_{0}}\right\|_{L^{\varphi}(\mathcal{X})}^{-1}\right)<\frac{\widetilde{C}_{0} \sigma \varphi\left(B_{k},\left\|\mathbf{1}_{B_{0}}\right\|_{L^{\varphi}(\mathcal{X})}^{-1}\right)}{s \mu\left(B_{k}\right)}\right\},\left\|\mathbf{1}_{B_{0}}\right\|_{L^{\varphi}(\mathcal{X})}^{-1}\right) \\
& \leq \sum_{k \in I} C_{3}\left(\frac{\widetilde{C}_{0} \sigma}{s}\right)^{q^{\prime}} \varphi\left(B_{k},\left\|\mathbf{1}_{B_{0}}\right\|_{L^{\varphi}(\mathcal{X})}^{-1}\right) \leq C_{3}\left(\frac{\widetilde{C}_{0} \sigma}{s}\right)^{q^{\prime}} \frac{\widetilde{C}_{1}}{\sigma} \varphi\left(B,\left\|\mathbf{1}_{B_{0}}\right\|_{L^{\varphi}(\mathcal{X})}^{-1}\right),
\end{aligned}
$$

where $C_{3}$ is the same fixed positive constant $C$ as in Lemma 3.2.

From this, (3.8) and (3.9), it follows that, for any $\sigma \in\left[\widetilde{C}_{1}, t\right], s \in(0, t)$ and $B \subset B_{0}$,

$$
\lambda(t, B) \leq\left[\frac{\widetilde{C}_{1}}{\sigma} \mathcal{F}(t-s)+C_{3}\left(\frac{\widetilde{C}_{0} \sigma}{s}\right)^{q^{\prime}} \frac{\widetilde{C}_{1}}{\sigma}\right] \varphi\left(B,\left\|\mathbf{1}_{B_{0}}\right\|_{L^{\varphi}(\mathcal{X})}^{-1}\right) .
$$

Then, by (3.12) with $\sigma:=4^{q^{\prime}} \widetilde{C}_{1}, s:=\frac{t}{2}$ and $C_{0}:=\max \left\{\sigma, \widetilde{C}_{1} C_{3}\left(2 \widetilde{C}_{0}\right)^{q^{\prime}} \sigma^{q^{\prime}-1}\right\}$, we have, for any $t \in\left(C_{0}, \infty\right)$,

$$
\mathcal{F}(t) \leq 4^{-q^{\prime}} \mathcal{F}\left(\frac{t}{2}\right)+C_{0} t^{-q^{\prime}} .
$$

Now, we show that, if $t \in\left(C_{0}, 2 C_{0}\right]$ and $m \in \mathbf{Z}_{+}$, then

$$
\mathcal{F}\left(2^{m} t\right) \leq\left(2 C_{0}\right)^{q^{\prime}}\left(2^{m} t\right)^{-q^{\prime}} .
$$

To this end, we use mathematical induction. Indeed, when $m=0$, we observe that

$$
\mathcal{F}\left(2^{m} t\right) \leq 1 \leq\left(2 C_{0}\right)^{q^{\prime}} t^{-q^{\prime}}
$$

which implies (3.14) in this case.

Suppose that (3.14) holds true with $m$ replaced by $m-1$. Then, from (3.13), we deduce that

$$
\begin{aligned}
\mathcal{F}\left(2^{m} t\right) & \leq 4^{-q^{\prime}} \mathcal{F}\left(2^{m-1} t\right)+C_{0}\left(2^{m} t\right)^{-q^{\prime}} \leq 4^{-q^{\prime}}\left(2 C_{0}\right)^{q^{\prime}}\left(2^{m-1} t\right)^{-q^{\prime}}+C_{0}\left(2^{m} t\right)^{-q^{\prime}} \\
& =\left(2 C_{0}\right)^{q^{\prime}}\left(2^{m} t\right)^{-q^{\prime}}\left(2^{-q^{\prime}}+2^{-q^{\prime}} C_{0}^{1-q^{\prime}}\right),
\end{aligned}
$$

which, together with the fact that

$$
2^{-q^{\prime}}+2^{-q^{\prime}} C_{0}^{1-q^{\prime}}<2^{-q^{\prime}}+2^{-q^{\prime}}<1
$$

implies that (3.14) holds true for $m$.

Then, by mathematical induction on $m$, we further conclude that (3.14) holds true for any $m \in \mathbf{Z}_{+}$. Furthermore, from (3.14), we deduce that, if $t \in\left(C_{0}, \infty\right)$, then

$$
\mathcal{F}(t) \leq\left(2 C_{0}\right)^{q^{\prime}} t^{-q^{\prime}}
$$

which implies the desired conclusion of Theorem 3.1 in Case 2 and hence completes the proof of Theorem 3.1.

In what follows, let

$$
m(\varphi):=\lfloor\omega[q(\varphi) / i(\varphi)-1]\rfloor,
$$


where, for any $a \in \mathbf{R},\lfloor a\rfloor$ denotes the largest integer which is not bigger than $a, \omega$ is as in (1.4), $i(\varphi)$ is as in $(2.1)$ and

$$
q(\varphi):=\inf \left\{q \in[1, \infty): \varphi \in \mathbf{A}_{q}(\mathcal{X})\right\} .
$$

In the spirit of the corresponding Euclidean case in [43, Theorem 2.7], we obtain the following several equivalent characterizations of $\mathrm{BMO}^{\varphi}(\mathcal{X})$ on $\mathcal{X}$.

Theorem 3.4. Let $\varphi$ be a growth function as in Definition 2.5, $q \in\left[1,[q(\varphi)]^{\prime}\right)$ and $\epsilon \in\left(\omega\left[\frac{q(\varphi)}{i(\varphi)}-1\right], \infty\right)$, where $i(\varphi)$ and $q(\varphi)$ are, respectively, as in (2.1) and (3.16), and $1 / q(\varphi)+1 /[q(\varphi)]^{\prime}=1$. Then, for any $f \in L_{\text {loc }}^{1}(\mathcal{X})$, the following statements are mutually equivalent:

(i) $\|f\|_{\mathrm{BMO}^{\varphi}(\mathcal{X})}<\infty$;

(ii) $\|f\|_{\operatorname{Lip}_{\varphi, q}(\mathcal{X})}<\infty$;

(iii) $\|f\|_{\operatorname{Lip}_{\varphi, 1}^{\epsilon}(\mathcal{X})}:=\sup _{\left\{x_{0} \in \mathcal{X}, r \in(0, \infty), B:=B\left(x_{0}, r\right) \subset \mathcal{X}\right\}} \frac{r^{\omega+\epsilon}}{\left\|\mathbf{1}_{B}\right\|_{L^{\varphi}(\mathcal{X})}} \int_{\mathcal{X}} \frac{\left|f(x)-m_{B}(f)\right|}{r^{\omega+\epsilon}+\left[d\left(x, x_{0}\right)\right]^{\omega+\epsilon}} d \mu(x)$ $<\infty$, where $m_{B}(f)$ is as in $(2.3)$.

Moreover, the norms $\|\cdot\|_{\mathrm{BMO}^{\varphi}(\mathcal{X})},\|\cdot\|_{\operatorname{Lip}_{\varphi, q}(\mathcal{X})}$ and $\|\cdot\|_{\operatorname{Lip}_{\varphi, 1}^{\epsilon}(\mathcal{X})}$ are mutually equivalent.

Proof. From Theorem 3.1 and some arguments similar to those used in the proof of [43, Theorem 2.7], it follows that "(i) $\Longleftrightarrow$ (ii)" and, for any $f \in L_{\text {loc }}^{1}(\mathcal{X})$,

$$
\|f\|_{\operatorname{Lip}_{\varphi, 1}(\mathcal{X})} \sim\|f\|_{\operatorname{Lip}_{\varphi, q}(\mathcal{X})},
$$

where the positive equivalence constants are independent of $f$.

Now, we show that "(iii) $\Longleftrightarrow(\mathrm{i})$ ". It is clear that "(iii) $\Longrightarrow(\mathrm{i})$ " and, for any $f \in L_{\text {loc }}^{1}(\mathcal{X})$,

$$
\|f\|_{\operatorname{Lip}_{\varphi, 1}(\mathcal{X})} \lesssim\|f\|_{\operatorname{Lip}_{\varphi, 1}^{\epsilon}(\mathcal{X})} .
$$

Then we are left to show that "(i) $\Longrightarrow$ (iii)". Fix any $x_{0} \in \mathcal{X}, r \in(0, \infty)$ and ball $B:=B\left(x_{0}, r\right)$. For any $k \in \mathbf{Z}_{+}:=\{0\} \cup \mathbf{N}$, let $B_{k}:=2^{k} B$. Then we write

$$
\begin{aligned}
\mathrm{I}: & =\frac{r^{\omega+\epsilon}}{\left\|\mathbf{1}_{B}\right\|_{L^{\varphi}(\mathcal{X})}} \int_{\mathcal{X}} \frac{\left|f(x)-m_{B}(f)\right|}{r^{\omega+\epsilon}+\left[d\left(x, x_{0}\right)\right]^{\omega+\epsilon}} d \mu(x) \\
& =\frac{r^{\omega+\epsilon}}{\left\|\mathbf{1}_{B}\right\|_{L^{\varphi}(\mathcal{X})}} \int_{B} \frac{\left|f(x)-m_{B}(f)\right|}{r^{\omega+\epsilon}+\left[d\left(x, x_{0}\right)\right]^{\omega+\epsilon}} d \mu(x)+\sum_{k=1}^{\infty} \frac{r^{\omega+\epsilon}}{\left\|\mathbf{1}_{B}\right\|_{L^{\varphi}(\mathcal{X})}} \int_{B_{k} \backslash B_{k-1}} \ldots \\
& =: \mathrm{I}_{1}+\sum_{k=1}^{\infty} \mathrm{I}_{2, k},
\end{aligned}
$$

where

and, for any $k \in \mathbf{N}$,

$$
\mathrm{I}_{1}:=\frac{r^{\omega+\epsilon}}{\left\|\mathbf{1}_{B}\right\|_{L^{\varphi}(\mathcal{X})}} \int_{B} \frac{\left|f(x)-m_{B}(f)\right|}{r^{\omega+\epsilon}+\left[d\left(x, x_{0}\right)\right]^{\omega+\epsilon}} d \mu(x)
$$

$$
\mathrm{I}_{2, k}:=\frac{r^{\omega+\epsilon}}{\left\|\mathbf{1}_{B}\right\|_{L^{\varphi}(\mathcal{X})}} \int_{B_{k} \backslash B_{k-1}} \frac{\left|f(x)-m_{B}(f)\right|}{r^{\omega+\epsilon}+\left[d\left(x, x_{0}\right)\right]^{\omega+\epsilon}} d \mu(x) .
$$

Obviously, we have

$$
\mathrm{I}_{1} \leq \frac{1}{\left\|\mathbf{1}_{B}\right\|_{L^{\varphi}(\mathcal{X})}} \int_{B}\left|f(x)-m_{B}(f)\right| d \mu(x) \leq\|f\|_{\operatorname{Lip}_{\varphi, 1}(\mathcal{X})} .
$$

Moreover, observe that $\epsilon \in\left(\omega\left[\frac{q(\varphi)}{i(\varphi)}-1\right], \infty\right)$ implies that there exist $p_{0} \in(0, i(\varphi))$ and $q_{0} \in(q(\varphi), \infty)$ such that $\epsilon>\omega\left(\frac{q_{0}}{p_{0}}-1\right)$. Thus, $\varphi \in \mathbf{A}_{q_{0}}(\mathcal{X})$ and $\varphi$ is of uniformly lower 
type $p_{0}$. By some arguments similar to those used in the proof of [43, Theorem 2.7], we conclude that, for any $k \in \mathbf{N}$,

$$
\left\|\mathbf{1}_{B_{k}}\right\|_{L^{\varphi}(\mathcal{X})} \lesssim 2^{k \omega \frac{q_{0}}{p_{0}}}\left\|\mathbf{1}_{B}\right\|_{L^{\varphi}(\mathcal{X})}
$$

which, together with (1.4), further implies that, for any $k \in \mathbf{N}$,

$$
\begin{aligned}
& \mathrm{I}_{2, k} \lesssim 2^{-k(\omega+\epsilon)} \frac{1}{\left\|\mathbf{1}_{B}\right\|_{L^{\varphi}(\mathcal{X})}} \int_{B_{k}}\left|f(x)-m_{B}(f)\right| d \mu(x) \\
& \lesssim 2^{-k(\omega+\epsilon)} \frac{1}{\left\|\mathbf{1}_{B}\right\|_{L^{\varphi}(\mathcal{X})}}\left[\int_{B_{k}}\left|f(x)-m_{B_{k}}(f)\right| d \mu(x)\right. \\
& \left.+\mu\left(B_{k}\right)\left|m_{B_{k}}(f)-m_{B}(f)\right|\right] \\
& \lesssim 2^{-k(\omega+\epsilon)} \frac{1}{\left\|\mathbf{1}_{B}\right\|_{L^{\varphi}(\mathcal{X})}}\left[\int_{B_{k}}\left|f(x)-m_{B_{k}}(f)\right| d \mu(x)\right. \\
& \left.+\mu\left(B_{k}\right) \sum_{s=1}^{k}\left|m_{B_{s}}(f)-m_{B_{s-1}}(f)\right|\right] \\
& \lesssim 2^{-k(\omega+\epsilon)}\left[\frac{1}{\left\|\mathbf{1}_{B}\right\|_{L^{\varphi}(\mathcal{X})}} \int_{B_{k}}\left|f(x)-m_{B_{k}}(f)\right| d \mu(x)\right. \\
& \left.+\sum_{s=1}^{k} \frac{\mu\left(B_{k}\right)}{\mu\left(B_{s-1}\right)} \frac{1}{\left\|\mathbf{1}_{B}\right\|_{L^{\varphi}(\mathcal{X})}} \int_{B_{s-1}}\left|f(x)-m_{B_{s}}(f)\right| d \mu(x)\right] \\
& \lesssim 2^{-k(\omega+\epsilon)}\left[2^{k \omega \frac{q_{0}}{p_{0}}}\|f\|_{\operatorname{Lip}_{\varphi, 1}(\mathcal{X})}\right. \\
& \left.+\sum_{s=1}^{k} \frac{\mu\left(B_{k}\right)}{\mu\left(B_{s}\right)} \frac{1}{\left\|\mathbf{1}_{B}\right\|_{L^{\varphi}(\mathcal{X})}} \int_{B_{s}}\left|f(x)-m_{B_{s}}(f)\right| d \mu(x)\right] \\
& \lesssim 2^{-k(\omega+\epsilon)}\left[2^{k \omega \frac{q_{0}}{p_{0}}}\|f\|_{\operatorname{Lip}_{\varphi, 1}(\mathcal{X})}+\sum_{s=1}^{k} 2^{(k-s) \omega} 2^{s \omega \frac{q_{0}}{p_{0}}}\|f\|_{\operatorname{Lip}_{\varphi, 1}(\mathcal{X})}\right] \\
& \lesssim 2^{-k(\omega+\epsilon)}\left[2^{k \omega \frac{q_{0}}{p_{0}}}+2^{k \omega} \sum_{s=-\infty}^{k} 2^{s \omega\left(\frac{q_{0}}{p_{0}}-1\right)}\right]\|f\|_{\operatorname{Lip}_{\varphi, 1}(\mathcal{X})} \\
& \sim 2^{-k(\omega+\epsilon)} 2^{k \omega \frac{q_{0}}{p_{0}}}\|f\|_{\operatorname{Lip}_{\varphi, 1}(\mathcal{X})} \sim 2^{-k\left[\epsilon-\omega\left(\frac{q_{0}}{p_{0}}-1\right)\right]}\|f\|_{\operatorname{Lip}_{\varphi, 1}(\mathcal{X})} .
\end{aligned}
$$

From (3.17), (3.18), (3.20) and $\epsilon>\omega\left(\frac{q_{0}}{p_{0}}-1\right)$, we deduce that

$$
\mathrm{I} \lesssim\|f\|_{\operatorname{Lip}_{\varphi, 1}(\mathcal{X})}+\sum_{k=1}^{\infty} 2^{-k\left[\epsilon-\omega\left(\frac{q_{0}}{p_{0}}-1\right)\right]}\|f\|_{\operatorname{Lip}_{\varphi, 1}(\mathcal{X})} \lesssim\|f\|_{\operatorname{Lip}_{\varphi, 1}(\mathcal{X})}
$$

which implies that, for any $f \in \operatorname{Lip}_{\varphi, 1}(\mathcal{X})$,

$$
\|f\|_{\operatorname{Lip}_{\varphi, 1}^{\epsilon}(\mathcal{X})} \lesssim\|f\|_{\operatorname{Lip}_{\varphi, 1}(\mathcal{X})}
$$

This finishes the proof of "(i) $\Longrightarrow$ (iii)" and hence of Theorem 3.4.

Remark 3.5. By Theorem 3.4, we know that $\operatorname{Lip}_{\varphi, q}(\mathcal{X})$ is independent of the choice of $q \in\left[1,[q(\varphi)]^{\prime}\right.$, with $q(\varphi)$ as in (3.16), in the sense of equivalent norms. 


\section{Musielak-Orlicz Hardy spaces via various maximal functions}

In this section, we introduce Musielak-Orlicz Hardy spaces, $H^{+, \varphi}(\mathcal{X}), H_{\theta}^{\varphi}(\mathcal{X})$ and $H^{*, \varphi}(\mathcal{X})$, defined, respectively, via radial, nontangential and grand maximal functions and show that $H^{+, \varphi}(\mathcal{X}), H_{\theta}^{\varphi}(\mathcal{X})$ and $H^{*, \varphi}(\mathcal{X})$ are mutually identical with equivalent quasi-norms.

4.1. Calderón reproducing formulae. In this section, we first recall the important Calderón reproducing formulae established in [28]. Now, we first recall the following system of dyadic cubes given in [32, Theorem 2.2] (see also [2]), which was formulated in [27, Lemma 2.3].

Lemma 4.1. Fix constants $c_{0}, C_{0}$ and $\delta$ such that $0<c_{0} \leq C_{0}<\infty, \delta \in(0,1)$ and $12 A_{0}^{3} C_{0} \delta \leq c_{0}$. Suppose that a set of points, $\left\{z_{\alpha}^{k}: k \in \mathbf{Z}, \alpha \in \mathscr{A}_{k}\right\} \subset \mathcal{X}$ with $\mathscr{A}_{k}$ $(k \in \mathbf{Z})$ being a countable set of indices, has the following properties: for any $k \in \mathbf{Z}$,

(i) $d\left(z_{\alpha}^{k}, z_{\beta}^{k}\right) \geq c_{0} \delta^{k}$ when $\alpha \neq \beta$;

(ii) $\min _{\alpha \in \mathscr{A}_{k}} d\left(x, z_{\alpha}^{k}\right) \leq C_{0} \delta^{k}$ for any $x \in \mathcal{X}$.

(The existence of such a set of points is guaranteed by the Zorn lemma.) Then there exists a family of sets, $\left\{Q_{\alpha}^{k}: k \in \mathbf{Z}, \alpha \in \mathscr{A}_{k}\right\}$ (which is called the system of half-open dyadic cubes), satisfying

(iii) $\mathcal{X}=\bigcup_{\alpha \in \mathscr{A}_{k}} Q_{\alpha}^{k}$, where $\left\{Q_{\alpha}^{k}: \alpha \in \mathscr{A}_{k}\right\}$ are mutually disjoint;

(iv) if $\ell \geq k, \alpha \in \mathscr{A}_{k}$ and $\beta \in \mathscr{A}_{\ell}$, then either $Q_{\beta}^{\ell} \subset Q_{\alpha}^{k}$ or $Q_{\alpha}^{k} \cap Q_{\beta}^{\ell}=\emptyset$;

(v) for any $\alpha \in \mathscr{A}_{k}, B\left(z_{\alpha}^{k}, c_{\natural} \delta^{k}\right) \subset Q_{\alpha}^{k} \subset B\left(z_{\alpha}^{k}, C^{\natural} \delta^{k}\right)=: B\left(Q_{\alpha}^{k}\right)$, where $c_{\natural}:=$ $\left(3 A_{0}^{2}\right)^{-1} c_{0}, C^{\natural}:=2 A_{0} C_{0}$ and $z_{\alpha}^{k}$ is called the "center" of $Q_{\alpha}^{k}$.

In what follows, for any $k \in \mathbf{Z}$, let

$$
\mathcal{X}^{k}:=\left\{z_{\alpha}^{k}\right\}_{\alpha \in \mathscr{A}_{k}}, \quad \mathscr{G}_{k}:=\mathscr{A}_{k+1} \backslash \mathscr{A}_{k} \quad \text { and } \quad \mathcal{Y}^{k}:=\mathcal{X}^{k+1} \backslash \mathcal{X}^{k} .
$$

Then we recall the following notion of the approximation of the identity with exponential decay from [28, Definition 2.7]; see also [27, Definition 2.4].

Definition 4.2. A sequence $\left\{Q_{k}\right\}_{k \in \mathbf{Z}}$ of bounded linear integral operators on $L^{2}(\mathcal{X})$ is called an approximation of the identity with exponential decay (for short, exp-ATI) if there exist constants $C, \nu \in(0, \infty), a \in(0,1]$ and $\eta \in(0,1)$ such that, for any $k \in \mathbf{Z}$, the kernel of the operator $Q_{k}$, which is still denoted by $Q_{k}$, satisfies

(i) (the identity condition) $\sum_{k=-\infty}^{\infty} Q_{k}=I$ in $L^{2}(\mathcal{X})$, where $I$ is the identity operator on $L^{2}(\mathcal{X})$

(ii) (the size condition) for any $x, y \in \mathcal{X}$,

$$
\begin{aligned}
\left|Q_{k}(x, y)\right| \leq & C \frac{1}{\sqrt{V_{\delta^{k}}(x) V_{\delta^{k}}(y)}} \exp \left\{-\nu\left[\frac{d(x, y)}{\delta^{k}}\right]^{a}\right\} \\
& \cdot \exp \left\{-\nu\left[\frac{\max \left\{d\left(x, \mathcal{Y}^{k}\right), d\left(y, \mathcal{Y}^{k}\right)\right\}}{\delta^{k}}\right]^{a}\right\}
\end{aligned}
$$

(iii) (the regularity condition) for any $x, \widetilde{x}, y \in \mathcal{X}$ with $d(x, \widetilde{x}) \leq \delta^{k}$,

$$
\begin{aligned}
& \left|Q_{k}(x, y)-Q_{k}(\widetilde{x}, y)\right|+\left|Q_{k}(y, x)-Q_{k}(y, \widetilde{x})\right| \\
& \leq C\left[\frac{d(x, \widetilde{x})}{\delta^{k}}\right]^{\eta} \frac{1}{\sqrt{V_{\delta^{k}}(x) V_{\delta^{k}}(y)}} \exp \left\{-\nu\left[\frac{d(x, y)}{\delta^{k}}\right]^{a}\right\} \\
& \quad \cdot \exp \left\{-\nu\left[\frac{\max \left\{d\left(x, \mathcal{Y}^{k}\right), d\left(y, \mathcal{Y}^{k}\right)\right\}}{\delta^{k}}\right]^{a}\right\}
\end{aligned}
$$


(iv) (the second difference regularity condition) for any $x, \widetilde{x}, y, \widetilde{y} \in \mathcal{X}$ with $d(x, \widetilde{x}) \leq$ $\delta^{k}$ and $d(y, \widetilde{y}) \leq \delta^{k}$,

$$
\begin{aligned}
& \left|\left[Q_{k}(x, y)-Q_{k}(\widetilde{x}, y)\right]-\left[Q_{k}(x, \widetilde{y})-Q_{k}(\widetilde{x}, \widetilde{y})\right]\right| \\
& \leq C\left[\frac{d(x, \widetilde{x})}{\delta^{k}}\right]^{\eta}\left[\frac{d(y, \widetilde{y})}{\delta^{k}}\right]^{\eta} \frac{1}{\sqrt{V_{\delta^{k}}(x) V_{\delta^{k}}(y)}} \exp \left\{-\nu\left[\frac{d(x, y)}{\delta^{k}}\right]^{a}\right\} \\
& \quad \cdot \exp \left\{-\nu\left[\frac{\max \left\{d\left(x, \mathcal{Y}^{k}\right), d\left(y, \mathcal{Y}^{k}\right)\right\}}{\delta^{k}}\right]^{a}\right\} ;
\end{aligned}
$$

(v) (the cancellation condition) for any $x, y \in \mathcal{X}$,

$$
\int_{\mathcal{X}} Q_{k}(x, \widetilde{y}) d \mu(\widetilde{y})=0=\int_{\mathcal{X}} Q_{k}(\widetilde{x}, y) d \mu(\widetilde{x}) .
$$

Via the set of points, $\left\{z_{\alpha}^{k}\right\}_{k \in \mathbf{Z}, \alpha \in \mathscr{A}_{k}}$, constructing random dyadic cubes (based on the system of dyadic cubes as in Lemma 4.1) and the spline functions, Auscher and Hytönen [2, Theorem 7.1] constructed the following system $\left\{\psi_{\beta}^{k}\right\}_{k \in \mathbf{Z}, \beta \in \mathscr{G}_{k}}$ of wavelet functions on $\mathcal{X}$, which is an orthonormal basis of $L^{2}(\mathcal{X})$ (see also [28, Theorem 2.6]).

Theorem 4.3. There exist constants $a \in(0,1], \eta \in(0,1), C, \nu \in(0, \infty)$ and wavelet functions $\left\{\psi_{\beta}^{k}\right\}_{k \in \mathbf{Z}, \beta \in \mathscr{G}_{k}}$ satisfying

(i) for any $k \in \mathbf{Z}, \beta \in \mathscr{G}_{k}$ and $x \in \mathcal{X}$,

$$
\left|\psi_{\beta}^{k}(x)\right| \leq C \frac{1}{\sqrt{V\left(y_{\beta}^{k}, \delta^{k}\right)}} \exp \left(-\nu\left[\frac{d\left(y_{\beta}^{k}, x\right)}{\delta^{k}}\right]^{a}\right)
$$

(ii) for any $k \in \mathbf{Z}, \beta \in \mathscr{G}_{k}$ and $x, y \in \mathcal{X}$ such that $d(x, y) \leq \delta^{k}$,

$$
\left|\psi_{\beta}^{k}(x)-\psi_{\beta}^{k}(y)\right| \leq C\left[\frac{d(x, y)}{\delta^{k}}\right]^{\eta} \frac{1}{\sqrt{V\left(y_{\beta}^{k}, \delta^{k}\right)}} \exp \left(-\nu\left[\frac{d\left(y_{\beta}^{k}, x\right)}{\delta^{k}}\right]^{a}\right)
$$

(iii) for any $k \in \mathbf{Z}$ and $\beta \in \mathscr{G}_{k}$,

$$
\int_{\mathcal{X}} \psi_{\beta}^{k}(x) d \mu(x)=0 .
$$

Moreover, the wavelets $\left\{\psi_{\beta}^{k}\right\}_{k \in \mathbf{Z}, \beta \in \mathscr{G}_{k}}$ form an orthonormal basis of $L^{2}(\mathcal{X})$ and an unconditional basis of $L^{p}(\mathcal{X})$ with any given $p \in(1, \infty)$.

Remark 4.4. (i) Let $\left\{\psi_{\beta}^{k}\right\}_{k \in \mathbf{Z}, \beta \in \mathscr{G}_{k}}$ be as in Theorem 4.3. For any $k \in \mathbf{Z}$ and $x, y \in \mathcal{X}$, letting

$$
D_{k}(x, y):=\sum_{\beta \in \mathscr{G}_{k}} \psi_{\beta}^{k}(x) \psi_{\beta}^{k}(y),
$$

it was shown in $\left[28\right.$, p. 291] that the sequence $\left\{D_{k}\right\}_{k \in \mathbf{Z}}$ satisfies all conditions (i) through (v) of Definition 4.2.

(ii) The importance of the index $\eta$ in Theorem 4.3 (and hence in Definition 4.2) lies not only in the regularity of the wavelet functions but also in the restriction of the index $p$ for all results on Hardy spaces $H^{p}(\mathcal{X})$; see, for instance, [27, Theorems 3.5, 4.2, 5.10, 6.3 and 7.3].

The following homogeneous continuous Calderón reproducing formula was established in [28, Theorem 4.15]. 
Theorem 4.5. Let $\left\{Q_{k}\right\}_{k \in \mathbf{Z}}$ be an exp-ATI and $\varrho, \vartheta \in(0, \eta)$. Then there exists a sequence $\left\{\widetilde{Q}_{k}\right\}_{k \in \mathbf{Z}}$ of bounded linear operators on $L^{2}(\mathcal{X})$ such that, for any $f$ in $\left(\stackrel{\mathcal{G}}{0}_{0}^{\eta}(\varrho, \vartheta)\right)^{\prime}\left[\right.$ resp., $L^{p}(\mathcal{X})$ with any given $\left.p \in(1, \infty)\right]$,

$$
f=\sum_{k=-\infty}^{\infty} \widetilde{Q}_{k} Q_{k} f
$$

where the series converges in $\left(\stackrel{\mathcal{G}}{0}_{0}^{\eta}(\varrho, \vartheta)\right)^{\prime}$ [resp., $L^{p}(\mathcal{X})$ with any given $\left.p \in(1, \infty)\right]$. Moreover, there exists a positive constant $C$ such that, for any $k \in \mathbf{Z}$, the kernel of $\widetilde{Q}_{k}$, which is still denoted by $\widetilde{Q}_{k}$, satisfies the following conditions:

(i) for any $x, y \in \mathcal{X}$,

$$
\left|\widetilde{Q}_{k}(x, y)\right| \leq C \frac{1}{V_{\delta^{k}}(x)+V(x, y)}\left[\frac{\delta^{k}}{\delta^{k}+d(x, y)}\right]^{\vartheta} ;
$$

(ii) for any $x, \widetilde{x}, y \in \mathcal{X}$ with $d(x, \widetilde{x}) \leq\left(2 A_{0}\right)^{-1}\left[\delta^{k}+d(x, y)\right]$,

$$
\left|\widetilde{Q}_{k}(x, y)-\widetilde{Q}_{k}(\widetilde{x}, y)\right| \leq C\left[\frac{d(x, \widetilde{x})}{\delta^{k}+d(x, y)}\right]^{\varrho} \frac{1}{V_{\delta^{k}}(x)+V(x, y)}\left[\frac{\delta^{k}}{\delta^{k}+d(x, y)}\right]^{\vartheta} ;
$$

(iii) for any $x \in \mathcal{X}$,

$$
\int_{\mathcal{X}} \widetilde{Q}_{k}(x, y) d \mu(y)=0=\int_{\mathcal{X}} \widetilde{Q}_{k}(y, x) d \mu(y) .
$$

Next we recall the following notion of 1-exp-ATIs from [27, Definition 2.8].

Definition 4.6. A sequence $\left\{P_{k}\right\}_{k \in \mathbf{Z}}$ of bounded linear integral operators on $L^{2}(\mathcal{X})$ is called an approximation of the identity with exponential decay and integration 1 (for short, 1-exp-ATI) if $\left\{P_{k}\right\}_{k \in \mathbf{Z}}$ has the following properties:

(i) for any $k \in \mathbf{Z}, P_{k}$ satisfies (ii), (iii) and (iv) of Definition 4.2 but without the exponential decay factor

$$
\exp \left\{-\nu\left[\frac{\max \left\{d\left(x, \mathcal{Y}^{k}\right), d\left(y, \mathcal{Y}^{k}\right)\right\}}{\delta^{k}}\right]^{a}\right\}
$$

where $\mathcal{Y}^{k}$ is as in (4.1);

(ii) for any $k \in \mathbf{Z}$ and $x \in \mathcal{X}, \int_{\mathcal{X}} P_{k}(x, y) d \mu(y)=1=\int_{\mathcal{X}} P_{k}(y, x) d \mu(y)$;

(iii) for any $k \in \mathbf{Z}$, letting $Q_{k}:=P_{k}-P_{k-1}$, then $\left\{Q_{k}\right\}_{k \in \mathbf{Z}}$ is an exp-ATI.

Remark 4.7. The existence of the 1-exp-ATI is guaranteed by [2, Lemma 10.1] (see also [27, Remark 2.9]).

4.2. Equivalences of Musielak-Orlicz Hardy spaces via various maximal functions. In this subsection, we show that $H^{+, \varphi}(\mathcal{X}), H_{\theta}^{\varphi}(\mathcal{X})$ and $H^{*, \varphi}(\mathcal{X})$ mutually coincide with equivalent quasi-norms.

We first introduce the notions of Musielak-Orlicz Hardy spaces defined via various maximal functions, which are generalizations of the corresponding spaces on Hardy spaces $H^{p}(\mathcal{X})$ in [27, Section 3] [in this case,

$$
\varphi(x, t):=t^{p} \quad \text { for any }(x, t) \in \mathcal{X} \times(0, \infty) \text { and any given } p \in(\omega /(\omega+\eta), 1]
$$

with $\omega$ as in (1.4) and $\eta$ as in Definition 4.2]. 
Definition 4.8. Let $\varrho, \vartheta \in(0, \eta)$ and $f \in\left(\mathcal{G}_{0}^{\eta}(\varrho, \vartheta)\right)^{\prime}$ with $\eta$ as in Definition 4.2. Let $\left\{P_{k}\right\}_{k \in \mathbf{Z}}$ be an 1-exp-ATI. The radial maximal function $\mathcal{M}^{+}(f)$ of $f$ is defined by setting

$$
\mathcal{M}^{+}(f)(x):=\sup _{k \in \mathbf{Z}}\left|P_{k} f(x)\right|, \quad \forall x \in \mathcal{X} .
$$

The non-tangential maximal function $\mathcal{M}_{\theta}(f)$ of $f$, with aperture $\theta \in(0, \infty)$, is defined by setting

$$
\mathcal{M}_{\theta}(f)(x):=\sup _{k \in \mathbf{Z}} \sup _{y \in B\left(x, \theta \delta^{k}\right)}\left|P_{k} f(y)\right|, \quad \forall x \in \mathcal{X} .
$$

Moreover, the grand maximal function $f^{*}$ of $f$ is defined by setting, for any $x \in \mathcal{X}$,

$$
f^{*}(x):=\sup \left\{|\langle f, h\rangle|: h \in \mathcal{G}_{0}^{\eta}(\varrho, \vartheta),\|h\|_{\mathcal{G}(x, r, \varrho, \vartheta)} \leq 1 \text { for some } r \in(0, \infty)\right\} .
$$

Let $\varphi$ be a growth function as in Definition 2.5. Then the Musielak-Orlicz Hardy spaces, $H^{+, \varphi}(\mathcal{X}), H_{\theta}^{\varphi}(\mathcal{X})$ [with $\left.\theta \in(0, \infty)\right]$ and $H^{*, \varphi}(\mathcal{X})$, are defined, respectively, by setting

$$
\begin{aligned}
H^{+, \varphi}(\mathcal{X}) & :=\left\{f \in\left(\mathcal{G}_{0}^{\eta}(\varrho, \vartheta)\right)^{\prime}:\|f\|_{H^{+, \varphi}(\mathcal{X})}:=\left\|\mathcal{M}^{+}(f)\right\|_{L^{\varphi}(\mathcal{X})}<\infty\right\}, \\
H_{\theta}^{\varphi}(\mathcal{X}) & :=\left\{f \in\left(\mathcal{G}_{0}^{\eta}(\varrho, \vartheta)\right)^{\prime}:\|f\|_{H_{\theta}^{\varphi}(\mathcal{X})}:=\left\|\mathcal{M}_{\theta}(f)\right\|_{L^{\varphi}(\mathcal{X})}<\infty\right\}
\end{aligned}
$$

and

$$
H^{*, \varphi}(\mathcal{X}):=\left\{f \in\left(\mathcal{G}_{0}^{\eta}(\varrho, \vartheta)\right)^{\prime}:\|f\|_{H^{*, \varphi}(\mathcal{X})}:=\left\|f^{*}\right\|_{L^{\varphi}(\mathcal{X})}<\infty\right\} .
$$

We first give the following property of $H^{*, \varphi}(\mathcal{X})$ on $\mathcal{X}$, which is a generalization of the Euclidean case in [35, Proposition 5.1].

Lemma 4.9. Let $\varphi, \varrho$ and $\vartheta$ be as in Definition 4.8 and $\eta$ as in Definition 4.2. Then $H^{*, \varphi}(\mathcal{X})$ continuously embeds into $\left(\mathcal{G}_{0}^{\eta}(\varrho, \vartheta)\right)^{\prime}$, namely, there exists a positive constant $C$ such that, for any $f \in H^{*, \varphi}(\mathcal{X})$ and $h \in \mathcal{G}_{0}^{\eta}(\varrho, \vartheta)$,

$$
|\langle f, h\rangle| \leq C\|h\|_{\mathcal{G}_{0}^{\eta}(\varrho, \vartheta)}\|f\|_{H^{*, \varphi}(\mathcal{X})}
$$

Proof. Let $h \in \mathcal{G}_{0}^{\eta}(\varrho, \vartheta)$. Then it is obvious that, for any $x \in B\left(x_{1}, 1\right)$,

$$
\|h\|_{\mathcal{G}_{0}^{\eta}(x, 1, \varrho, \vartheta)} \lesssim\|h\|_{\mathcal{G}_{0}^{\eta}\left(x_{1}, 1, \varrho, \vartheta\right)} \sim\|h\|_{\mathcal{G}_{0}^{\eta}(\varrho, \vartheta)},
$$

where $x_{1} \in \mathcal{X}$ is as in Definition 2.1. Thus, for any $x \in B\left(x_{1}, 1\right)$,

$$
|\langle f, h\rangle| \lesssim\|h\|_{\mathcal{G}_{0}^{\eta}(\varrho, \vartheta)} f^{*}(x),
$$

which further implies that

$$
|\langle f, h\rangle| \lesssim\|h\|_{\mathcal{G}_{0}^{\eta}(\varrho, \vartheta)} \inf _{x \in B\left(x_{1}, 1\right)} f^{*}(x) \lesssim\|h\|_{\mathcal{G}_{0}^{\eta}(\varrho, \vartheta)}\left\|\mathbf{1}_{B\left(x_{1}, 1\right)}\right\|_{L^{\varphi}(\mathcal{X})}^{-1}\|f\|_{H^{*, \varphi}(\mathcal{X})} .
$$

This finishes the proof of Lemma 4.9 .

Remark 4.10. (i) It was shown in $[27,(3.1)]$ that, for any $f \in\left(\mathcal{G}_{0}^{\eta}(\varrho, \vartheta)\right)^{\prime}$ and $x \in \mathcal{X}$,

$$
\mathcal{M}^{+} f(x) \leq \mathcal{M}_{\theta}(f)(x) \lesssim f^{*}(x),
$$

where the implicit positive constant only depends on $\theta$.

(ii) Similarly to the Euclidean case in [35, Propsition 5.2], by Lemma 4.9, we can show that $H^{*, \varphi}(\mathcal{X})$ is complete; the details are omitted. 
The following boundedness of the Hardy-Littlewood maximal operator $M$ as in $(2.2)$ on $L^{\varphi}(\mathcal{X})$ is similar to the Euclidean case in [41, Corollary 2.8] (see also [59, Corollary 2.1.2]); the details are omitted.

Theorem 4.11. Assume that $\varphi$ is a Musielak-Orlicz function of uniformly lower type $p_{\varphi}^{-}$and of uniformly upper type $p_{\varphi}^{+}$with $q(\varphi)<p_{\varphi}^{-} \leq p_{\varphi}^{+}<\infty$, where $q(\varphi)$ is as in (3.16). Then the Hardy-Littlewood maximal function $M$ is bounded on $L^{\varphi}(\mathcal{X})$ and, moreover, there exists a positive constant $C$ such that, for any $f \in L^{\varphi}(\mathcal{X})$,

$$
\int_{\mathcal{X}} \varphi(x, M(f)(x)) d \mu(x) \leq C \int_{\mathcal{X}} \varphi(x,|f(x)|) d \mu(x) .
$$

Now, we state the main result of this section, which is a generalization of the corresponding results on Hardy spaces $H^{p}(\mathcal{X})$ in [27, Theorem 3.5] [in this case, $\varphi$ is the same as in (4.2)].

Theorem 4.12. Assume that $\varphi$ is a growth function as in Definition 2.5 and $\theta \in(0, \infty)$. Then, for any $f \in\left(\mathcal{G}_{0}^{\eta}(\varrho, \vartheta)\right)^{\prime}$ with $\varrho, \vartheta \in\left(\omega\left[\frac{q(\varphi)}{p}-1\right], \eta\right)$ with $\omega$ as in (1.4), $q(\varphi)$ as in (3.16), $p$ as in Definition 2.5 and $\eta$ as in Definition 4.2,

$$
\|f\|_{H^{+, \varphi}(\mathcal{X})} \sim\|f\|_{H_{\theta}^{\varphi}(\mathcal{X})} \sim\|f\|_{H^{*, \varphi}(\mathcal{X})},
$$

with positive equivalence constants independent of $f$.

Proof. Let $f \in\left(\mathcal{G}_{0}^{\eta}(\varrho, \vartheta)\right)^{\prime}$ with $\varrho, \vartheta \in\left(\omega\left[\frac{q(\varphi)}{p}-1\right], \eta\right)$. Fix $\theta \in(0, \infty)$. From Remark 4.10(i), it follows that

$$
\left\|\mathcal{M}^{+}(f)\right\|_{L^{\varphi}(\mathcal{X})} \lesssim\left\|\mathcal{M}_{\theta}(f)\right\|_{L^{\varphi}(\mathcal{X})} \lesssim\left\|f^{*}\right\|_{L^{\varphi}(\mathcal{X})} .
$$

Thus, to complete the proof of Theorem 4.12, it suffices to show that, for any $f \in$ $\left(\mathcal{G}_{0}^{\eta}(\varrho, \vartheta)\right)^{\prime}$ with $\varrho, \vartheta \in\left(\omega\left[\frac{q(\varphi)}{p}-1\right], \eta\right)$,

$$
\left\|f^{*}\right\|_{L^{\varphi}(\mathcal{X})} \lesssim\left\|\mathcal{M}^{+}(f)\right\|_{L^{\varphi}(\mathcal{X})}
$$

To show (4.4), we first recall the following estimate in $[27,(3.5)]$ that, for any given $r \in\left(0, \frac{p}{q(\varphi)}\right) \subset(0, p)$ and, for any $f \in\left(\mathcal{G}_{0}^{\eta}(\varrho, \vartheta)\right)^{\prime}$ with $\varrho, \vartheta \in\left(\omega\left[\frac{q(\varphi)}{p}-1\right], \eta\right)$ and any $x \in \mathcal{X}$,

$$
f^{*}(x) \lesssim \mathcal{M}^{+}(f)(x)+\left\{M\left(\left[\mathcal{M}^{+}(f)\right]^{r}\right)(x)\right\}^{1 / r}
$$

By this and the boundedness of $M$ on $L^{\widetilde{\varphi}}(\mathcal{X})$ with $\widetilde{\varphi}(x, t):=\varphi\left(x, t^{1 / r}\right)$ for any $x \in \mathcal{X}$ and $t \in(0, \infty)$ (see Theorem 4.11), we conclude that

$$
\left\|f^{*}\right\|_{L^{\varphi}(\mathcal{X})} \lesssim\left\|\mathcal{M}^{+}(f)\right\|_{L^{\varphi}(\mathcal{X})}+\left\|M\left(\left[\mathcal{M}^{+}(f)\right]^{r}\right)\right\|_{L^{\tilde{\varphi}}(\mathcal{X})}^{1 / r} \sim\left\|\mathcal{M}^{+}(f)\right\|_{L^{\varphi}(\mathcal{X})},
$$

where we used the fact that $\widetilde{\varphi}$ is of uniformly lower type $p / r>q(\varphi)$ when $r \in\left(0, \frac{p}{q(\varphi)}\right)$. This implies (4.4) and hence finishes the proof of Theorem 4.12.

\section{Atomic characterizations of $H^{*, \varphi}(\mathcal{X})$}

This section is devoted to the atomic characterizations of $H^{*, \varphi}(\mathcal{X})$, which is divided into three parts.

We need the following affiliated spaces, which are generalizations of the corresponding Euclidean case in [35, Definition 2.3] to any space of homogeneous type. 
Definition 5.1. For any measurable set $E$ in $\mathcal{X}$, the space $L_{\varphi}^{q}(E)$ for any given $q \in[1, \infty]$ is defined to be the set of all measurable functions $f$ on $\mathcal{X}$, supported in $E$, such that

$$
\|f\|_{L_{\varphi}^{q}(E)}:= \begin{cases}\sup _{t \in(0, \infty)}\left[\frac{1}{\varphi(E, t)} \int_{\mathcal{X}}|f(x)|^{q} \varphi(x, t) d \mu(x)\right]^{1 / q}<\infty & \text { when } q \in[1, \infty), \\ \|f\|_{L^{\infty}(\mathcal{X})}<\infty & \text { when } q=\infty .\end{cases}
$$

We then introduce the notions of (finite) atomic Musielak-Orlicz Hardy spaces on spaces of homogeneous type, which are generalizations of the corresponding Euclidean case in [35, Definition 2.4].

Definition 5.2. A couple $(\varphi, q)$ is said to be admissible if $q \in(q(\varphi), \infty]$ and $m(\varphi) \leq 0$, where $q(\varphi)$ is as in (3.16) and $m(\varphi)$ as in (3.15). A measurable function $a$ is called a $(\varphi, q)$-atom supported in a ball $B \subset \mathcal{X}$ if the following three conditions hold true:

(i) $\operatorname{supp} a:=\{x \in \mathcal{X}: a(x) \neq 0\} \subset B$;

(ii) $a \in L_{\varphi}^{q}(B)$ and $\|a\|_{L_{\varphi}^{q}(B)} \leq\left\|\mathbf{1}_{B}\right\|_{L^{\varphi}(\mathcal{X})}^{-1}$;

(iii) $\int_{\mathcal{X}} a(x) d \mu(x)=0$.

The atomic Musielak-Orlicz Hardy space $H_{\mathrm{at}}^{\varphi, q}(\mathcal{X})$ is defined to be the set of all distributions $f \in\left(\mathcal{G}_{0}^{\eta}(\varrho, \vartheta)\right)^{\prime}$ with

$$
\varrho, \vartheta \in\left(\omega\left[\frac{q(\varphi)}{p}-1\right], \eta\right)
$$

here $\omega$ is as in (1.4), $p$ as in Definition 2.5 and $\eta$ as in Definition 4.2, satisfying that there exists a sequence $\left\{b_{j}\right\}_{j \in \mathbf{N}}$ of multiples of some $(\varphi, q)$-atoms supported, respectively, in balls $\left\{B_{j}\right\}_{j \in \mathbf{N}}$, such that

$$
\sum_{j=1}^{\infty} \varphi\left(B_{j},\left\|b_{j}\right\|_{L_{\varphi}^{q}\left(B_{j}\right)}\right)<\infty
$$

and $f=\sum_{j=1}^{\infty} b_{j}$ in $\left(\mathcal{G}_{0}^{\epsilon}(\varrho, \vartheta)\right)^{\prime}$. Moreover, let

$$
\Lambda_{q}\left(\left\{b_{j}\right\}_{j=1}^{\infty}\right):=\inf \left\{\lambda \in(0, \infty): \sum_{j=1}^{\infty} \varphi\left(B_{j}, \frac{\left\|b_{j}\right\|_{L_{\varphi}^{q}\left(B_{j}\right)}}{\lambda}\right) \leq 1\right\}
$$

and

$$
\|f\|_{H_{\mathrm{at}}^{\varphi, q}(\mathcal{X})}:=\inf \left\{\Lambda_{q}\left(\left\{b_{j}\right\}_{j=1}^{\infty}\right): f=\sum_{j=1}^{\infty} b_{j} \quad \text { in } \quad\left(\mathcal{G}_{0}^{\eta}(\varrho, \vartheta)\right)^{\prime}\right\}
$$

where the last infimum is taken over all decompositions of $f$ as above.

The finite atomic Musielak-Orlicz Hardy space $H_{\text {fin }}^{\varphi, q}(\mathcal{X})$ is defined to be the set of all finite linear combinations $f$ of $(\varphi, q)$-atoms, namely, for some $N \in \mathbf{N}$,

$$
f=\sum_{j=1}^{N} b_{j}
$$


where $\left\{b_{j}\right\}_{j=1}^{N}$ are multiples of some $(\varphi, q)$-atoms supported, respectively, in balls $\left\{B_{j}\right\}_{j=1}^{N}$. Moreover, define

$$
\|f\|_{H_{\text {fin }}^{\varphi, q}(\mathcal{X})}:=\inf \left\{\Lambda_{q}\left(\left\{b_{j}\right\}_{j=1}^{N}\right): f=\sum_{j=1}^{N} b_{j}\right\}
$$

where the infimum is taken over all finite decompositions of $f$ as above.

Remark 5.3. It is obvious that $m(\varphi) \leq 0$ is equivalent to $\omega q(\varphi)<(\omega+1) i(\varphi)$. Thus, the real-variable theory of $H_{\mathrm{at}}^{\varphi, q}(\mathcal{X})$ for the case $\omega q(\varphi) \geq(\omega+1) i(\varphi)$ [or, equivalently, $m(\varphi)>0$ ] is failed on $\mathcal{X}$, because the $(\varphi, q)$-atoms only have 0 -order cancelation.

Now, we are ready to state the main result of this section, which is a generalization of the corresponding results on Hardy spaces $H^{p}(\mathcal{X})$ in [27, Theorem 4.2] [in this case, $\varphi$ is the same as in (4.2)].

Theorem 5.4. Let $\varphi$ be a growth function, $m(\varphi) \leq 0$ with $m(\varphi)$ as in (3.15), $q \in(q(\varphi), \infty]$ and $\varrho, \vartheta$ as in (5.1) with $p>\frac{\omega}{\eta+\omega} q(\varphi)$. Then $H^{*, \varphi}(\mathcal{X})=H_{\text {at }}^{\varphi, q}(\mathcal{X})$ as subspaces of $\left(\mathcal{G}_{0}^{\eta}(\varrho, \vartheta)\right)^{\prime}$ with equivalent quasi-norms.

Remark 5.5. Due to Theorem 5.4, $H_{\mathrm{at}}^{\varphi, q}(\mathcal{X})$ is independent of the choice of $q \in(q(\varphi), \infty]$ in the sense of equivalent quasi-norms. In what follows, $H_{\mathrm{at}}^{\varphi, q}(\mathcal{X})$ is simply denoted by $H_{\mathrm{at}}^{\varphi}(\mathcal{X})$.

5.1. Proof of $\boldsymbol{H}_{\mathrm{at}}^{\varphi, q}(\mathcal{X}) \subset \boldsymbol{H}^{*, \varphi}(\mathcal{X})$. In this subsection, we show that $H_{\mathrm{at}}^{\varphi, q}(\mathcal{X}) \subset H^{*, \varphi}(\mathcal{X})$

Proof of Theorem 5.4, Part I. In this step, we show $H_{\mathrm{at}}^{\varphi, q}(\mathcal{X}) \subset H^{*, \varphi}(\mathcal{X})$. Let $b$ be a multiple of a $(\varphi, q)$-atom supported in a ball $B:=B\left(x_{B}, r_{B}\right)$ with $x_{B} \in \mathcal{X}$ and $r_{B} \in(0, \infty)$. We first claim that

$$
\int_{\mathcal{X}} \varphi\left(x, b^{*}(x)\right) d \mu(x) \lesssim \varphi\left(B,\|b\|_{L_{\varphi}^{q}(B)}\right) .
$$

Indeed, let $\widetilde{B}:=2 A_{0} B$. When $x \in \widetilde{B}$, from the proof of [27, (4.1)], it follows that $b^{*}(x) \lesssim M(b)(x)$. By Lemma 2.6(iii) and the fact that $\varphi \in \mathbf{A}_{q}(\mathcal{X})$ for any $q \in(q(\varphi), \infty)$, we conclude that, for any $t \in(0, \infty)$,

$$
\|b\|_{L^{1}(\mathcal{X})} \leq \mu(B)\left\{\frac{1}{\varphi(B, t)} \int_{B}|b(x)|^{q} \varphi(x, t) d \mu(x)\right\}^{1 / q} \leq \mu(B)\|b\|_{L_{\varphi}^{q}(B)},
$$

which, combined with some arguments used in the proof of [27, (4.1)], further implies that, for any $h \in \mathcal{G}_{0}^{\eta}(\varrho, \vartheta)$ with $\varrho, \vartheta \in\left(\omega\left[\frac{q(\varphi)}{p}-1\right], \eta\right)$, and $x \in(\widetilde{B})^{\complement}$,

$$
|\langle b, h\rangle| \leq\left[\frac{r_{B}}{d\left(x_{B}, x\right)}\right]^{\varrho} \frac{1}{V\left(x_{B}, x\right)}\|b\|_{L^{1}(\mathcal{X})} \leq\left[\frac{r_{B}}{d\left(x_{B}, x\right)}\right]^{\varrho} \frac{\mu(B)}{V\left(x_{B}, x\right)}\|b\|_{L_{\varphi}^{q}(B)} .
$$

Therefore, taking the supremum over all $h \in \mathcal{G}_{0}^{\eta}(\varrho, \vartheta)$ satisfying $\|h\|_{\mathcal{G}(x, r, \varrho, \vartheta)} \leq 1$ for some $r \in(0,1)$, we conclude that, for any $x \in \mathcal{X}$,

$$
b^{*}(x) \lesssim M(b) \mathbf{1}_{\widetilde{B}}(x)+\left[\frac{r_{B}}{d\left(x_{B}, x\right)}\right]^{\varrho} \frac{\mu(B)}{V\left(x_{B}, x\right)}\|b\|_{L_{\varphi}^{q}(B)} \mathbf{1}_{\widetilde{B}^{\complement}}(x) .
$$

Now, we use (5.3) to prove (5.2). To this end, let $q \in(q(\varphi), \infty]$. From the Hölder inequality and the uniformly upper type 1 property of $\varphi$, and (iv) and (v) of Lemma 2.6, 
we deduce that

$$
\begin{aligned}
& \int_{\widetilde{B}} \varphi\left(x, b^{*}(x)\right) d \mu(x) \\
& \lesssim \int_{\widetilde{B}} \varphi(x, M(b)(x)) d \mu(x) \lesssim \int_{\widetilde{B}}\left[\frac{M(b)(x)}{\|b\|_{L_{\varphi}^{q}(B)}}+1\right] \varphi\left(x,\|b\|_{L_{\varphi}^{q}(B)}\right) d \mu(x) \\
& \lesssim \varphi\left(\widetilde{B},\|b\|_{L_{\varphi}^{q}(B)}\right) \\
& +\frac{1}{\|b\|_{L_{\varphi}^{q}(B)}}\left\{\int_{\widetilde{B}}[M(b)(x)]^{q} \varphi\left(x,\|b\|_{L_{\varphi}^{q}(B)}\right) d \mu(x)\right\}^{\frac{1}{q}}\left[\varphi\left(\widetilde{B},\|b\|_{L_{\varphi}^{q}(B)}\right)\right]^{\frac{q-1}{q}} \\
& \lesssim\left[1+\frac{1}{\|b\|_{L_{\varphi}^{q}(B)}}\|b\|_{L_{\varphi}^{q}(B)}\right] \varphi\left(\widetilde{B},\|b\|_{L_{\varphi}^{q}(B)}\right) \lesssim \varphi\left(B,\|b\|_{L_{\varphi}^{q}(B)}\right) .
\end{aligned}
$$

On another hand, choose $q>q(\varphi)$ such that $\omega\left[\frac{q}{p}-1\right]<\eta$ and $\varrho \in\left(\omega\left[\frac{q}{p}-1\right], \eta\right)$. By (5.3), the uniformly lower type $p \in(0,1]$ property of $\varphi$, Lemma 2.6(iv) and $\varrho \in\left(\omega\left[\frac{q}{p}-1\right], \eta\right)$, we obtain

$$
\begin{aligned}
& \int_{\widetilde{B}^{\complement}} \varphi\left(x, b^{*}(x)\right) d \mu(x) \\
& \lesssim \int_{\widetilde{B}^{\complement}} \varphi\left(x,\left[\frac{r_{B}}{d\left(x_{B}, x\right)}\right]^{\varrho} \frac{\mu(B)}{V\left(x_{B}, x\right)}\|b\|_{L_{\varphi}^{q}(B)}\right) d \mu(x) \\
& \lesssim \sum_{k=1}^{\infty} \int_{2^{k+1} A_{0} B \backslash 2^{k} A_{0} B} \varphi\left(x, 2^{-k \varrho} \frac{\mu(B)}{V\left(x_{B}, 2^{k} r_{B}\right)}\|b\|_{L_{\varphi}^{q}(B)}\right) d \mu(x) \\
& \lesssim \sum_{k=1}^{\infty} 2^{-k \varrho p}\left[\frac{\mu(B)}{V\left(x_{B}, 2^{k} r_{B}\right)}\right]^{p} \int_{2^{k+1} A_{0} B \backslash 2^{k} A_{0} B} \varphi\left(x,\|b\|_{L_{\varphi}^{q}(B)}\right) d \mu(x) \\
& \lesssim \sum_{k=1}^{\infty} 2^{-k \varrho p}\left[\frac{\mu(B)}{V\left(x_{B}, 2^{k} r_{B}\right)}\right]^{p} \varphi\left(2^{k+1} A_{0} B,\|b\|_{L_{\varphi}^{q}(B)}\right) \\
& \lesssim \sum_{k=1}^{\infty} 2^{-k \varrho p}\left[\frac{\mu(B)}{V\left(x_{B}, 2^{k} r_{B}\right)}\right]^{p}\left[\frac{\mu\left(2^{k+1} A_{0} B\right)}{\mu(B)}\right]^{q} \varphi\left(B,\|b\|_{L_{\varphi}^{q}(B)}\right) \\
& \lesssim \sum_{k=1}^{\infty} 2^{-k \varrho p}\left[\frac{\mu\left(2^{k} B\right)}{\mu(B)}\right]^{q-p} \varphi\left(B,\|b\|_{L_{\varphi}^{q}(B)}\right) \\
& \lesssim \sum_{k=1}^{\infty} 2^{-k[\varrho p-\omega(q-p)]} \varphi\left(B,\|b\|_{L_{\varphi}^{q}(B)}\right) \lesssim \varphi\left(B,\|b\|_{L_{\varphi}^{q}(B)}\right) .
\end{aligned}
$$

Thus, we have

$$
\int_{\mathcal{X}} \varphi\left(x, b^{*}(x)\right) d \mu(x)=\int_{\widetilde{B}} \varphi\left(x, b^{*}(x)\right) d \mu(x)+\int_{\widetilde{B}^{\complement}} \cdots \lesssim \varphi\left(B,\|b\|_{L_{\varphi}^{q}(B)}\right)
$$

which completes the proof of the above claim (5.2). 
Moreover, for any $f \in H_{\mathrm{at}}^{\varphi, q}(\mathcal{X})$, by Definition 5.2, we know that $f \in\left(\mathcal{G}_{0}^{\eta}(\varrho, \vartheta)\right)^{\prime}$, with $\varrho, \vartheta \in\left(\omega\left[\frac{q(\varphi)}{p}-1\right], \eta\right)$, and there exists a sequence $\left\{b_{j}\right\}_{j=1}^{\infty}$ of multiples of $(\varphi, q)$ atoms such that

$$
f=\sum_{j=1}^{\infty} b_{j} \quad \text { in }\left(\mathcal{G}_{0}^{\eta}(\varrho, \vartheta)\right)^{\prime} \quad \text { and } \quad \Lambda_{q}\left(\left\{b_{j}\right\}_{j=1}^{\infty}\right) \lesssim\|f\|_{H_{\mathrm{at}}^{\varphi, q}(\mathcal{X})} .
$$

Thus, for any $x \in \mathcal{X}, f^{*}(x) \leq \sum_{j=1}^{\infty}\left(b_{j}\right)^{*}(x)$. From this, Lemma 2.8(i) and the above claim (5.2), it follows that

$$
\begin{aligned}
\int_{\mathcal{X}} \varphi\left(x, \frac{f^{*}(x)}{\Lambda_{q}\left(\left\{b_{j}\right\}_{j=1}^{\infty}\right)}\right) d \mu(x) & \lesssim \sum_{j=1}^{\infty} \int_{\mathcal{X}} \varphi\left(x, \frac{\left(b_{j}\right)^{*}(x)}{\Lambda_{q}\left(\left\{b_{j}\right\}_{j=1}^{\infty}\right)}\right) d \mu(x) \\
& \lesssim \sum_{j=1}^{\infty} \varphi\left(B_{j}, \frac{\left\|b_{j}\right\|_{L_{\varphi}^{q}(B)}}{\Lambda_{q}\left(\left\{b_{j}\right\}_{j=1}^{\infty}\right)}\right) \lesssim 1
\end{aligned}
$$

which further implies that

$$
\|f\|_{H^{*, \varphi}(\mathcal{X})} \sim\left\|f^{*}\right\|_{L^{\varphi}(\mathcal{X})} \lesssim \Lambda_{q}\left(\left\{b_{j}\right\}_{j=1}^{\infty}\right) \lesssim\|f\|_{H_{\mathrm{at}}^{\varphi, q}(\mathcal{X})}
$$

This finishes the proof of $H_{\mathrm{at}}^{\varphi, q}(\mathcal{X}) \subset H^{*, \varphi}(\mathcal{X})$ and hence of the Part I of the proof of Theorem 5.4.

5.2. Calderón-Zygmund decompositions of $\boldsymbol{H}^{*, \varphi}(\mathcal{X})$. We obtain a version of Calderón-Zygmund decompositions for $H^{*, \varphi}(\mathcal{X})$ in this subsection.

Suppose that $f \in H_{\mathrm{at}}^{\varphi, q}(\mathcal{X})$. Then, by Definition 5.2, we know that $f \in\left(\mathcal{G}_{0}^{\eta}(\varrho, \vartheta)\right)^{\prime}$ with $\varrho, \vartheta$ as in (5.1). Since the level set $\left\{x \in \mathcal{X}: f^{*}(x)>\lambda\right\}$ with $\lambda \in(0, \infty)$ may not be open even in the case that $d$ is a metric, to obtain the Calderón-Zygmund decomposition of $f$, we need to use some ideas from [49, Theorem 2] and [21, Remark 2.9] (see also [27, Section 4.2]).

From the proof of [49, Theorem 2], it follows that there exist a constant $\theta \in(0,1)$ and a metric $d^{\prime} \sim d^{\theta}$. For any $x \in \mathcal{X}$ and $r \in(0, \infty)$, define the $d^{\prime}$-ball $B^{\prime}(x, r):=$ $\left\{y \in \mathcal{X}: d^{\prime}(x, y)<r\right\}$. Then $\left(\mathcal{X}, d^{\prime}, \mu\right)$ is a doubling metric measure space. We also recall the following variant of the space of test functions associated with the metric $d^{\prime}$ from [27, Definition 4.6].

Definition 5.6. Let $x \in \mathcal{X}, r \in(0, \infty)$ and $\widetilde{\varrho}, \widetilde{\vartheta} \in(0, \infty)$. The space of test functions, denoted by $G(x, r, \widetilde{\varrho}, \widetilde{\vartheta})$, is defined to be the set of all functions $f$ on $\mathcal{X}$ satisfying that there exists a non-negative constant $C$ such that

$(\mathrm{T} 1)^{\prime}$ for any $y \in \mathcal{X},|f(y)| \leq C \frac{1}{\mu\left(B^{\prime}\left(y, r+d^{\prime}(x, y)\right)\right)}\left[\frac{r}{r+d^{\prime}(x, y)}\right]^{\widetilde{\vartheta}}$;

$(\mathrm{T} 2)^{\prime}$ for any $y, \widetilde{y} \in \mathcal{X}$ satisfying $d(y, \widetilde{y}) \leq\left[r+d^{\prime}(x, y)\right] / 2$,

$$
|f(y)-f(\widetilde{y})| \leq C\left[\frac{d^{\prime}(y, \widetilde{y})}{r+d^{\prime}(x, y)}\right]^{\widetilde{\varrho}} \frac{1}{\mu\left(B^{\prime}\left(y, r+d^{\prime}(x, y)\right)\right)}\left[\frac{r}{r+d^{\prime}(x, y)}\right]^{\widetilde{\vartheta}} .
$$

Moreover, for any $f \in G(x, r, \widetilde{\varrho}, \widetilde{\vartheta})$, define

$$
\|f\|_{G(x, r, \widetilde{\varrho}, \widetilde{\vartheta})}:=\inf \left\{C: C \text { satisfies }(\mathrm{T} 1)^{\prime} \text { and }(\mathrm{T} 2)^{\prime}\right\} \text {. }
$$

It was shown in $\left[27\right.$, Section 4.2] that $\mathcal{G}(x, r, \varrho, \vartheta)=G\left(x, r^{\theta}, \varrho / \theta, \vartheta / \theta\right)$ with equivalent norms. Moreover, for any $\varrho, \vartheta \in(0, \eta)$ and $f \in\left(\mathcal{G}_{0}^{\eta}(\varrho, \vartheta)\right)^{\prime}$, the modified grand maximal function $f^{\star}$ of $f$ is defined by setting, for any $x \in \mathcal{X}$,

$$
f^{\star}(x):=\sup \left\{\langle f, h\rangle: h \in \mathcal{G}_{0}^{\eta}(\varrho, \vartheta),\|h\|_{G(x, r, \varrho / \theta, \vartheta / \theta)} \leq 1 \text { for some } r \in(0, \infty)\right\} .
$$


Then we know that $f^{\star} \sim f^{*}$ pointwisely on $\mathcal{X}$ with the positive equivalence constants independent of $f$, where $f^{*}$ is as in Definition 4.8. For any $\lambda \in(0, \infty)$ and $j \in \mathbf{Z}$, define

$$
\Omega_{\lambda}:=\left\{x \in \mathcal{X}: f^{\star}(x)>\lambda\right\} \quad \text { and } \quad \Omega^{j}:=\Omega_{2^{j}} .
$$

It was shown in $\left[21\right.$, Remark 2.9(ii)] that, for any $\lambda \in(0, \infty), \Omega_{\lambda}$ is open under the topology induced by $d^{\prime}$ and hence under the topology induced by $d$.

Suppose that $\varrho, \vartheta \in\left(\omega\left[\frac{q(\varphi)}{p}-1\right], \eta\right)$ and $f \in H_{\mathrm{at}}^{\varphi, q}(\mathcal{X})$. Then $f^{\star} \in L^{\varphi}(\mathcal{X})$ and $\mu\left(\Omega_{j}\right)<\infty$ for any $j \in \mathbf{Z}$. Consequently, it was shown in [27, Section 4.2] that, for any $j \in \mathbf{Z}$, there exist $\left\{x_{k}^{j}\right\}_{k \in I_{j}}$ with $I_{j}$ being a countable index set, $\left\{r_{k}^{j}\right\}_{k \in I_{j}} \subset(0, \infty)$, $L_{0} \in \mathbf{N}$ and a sequence $\left\{\phi_{k}^{j}\right\}_{k \in I_{j}}$ of non-negative functions satisfying all conclusions of [27, Propositions 4.4 and 4.5]. For any $j \in \mathbf{Z}$ and $k \in I_{j}$, the operator $\Phi_{k}^{j}$ is defined by setting, for any $h \in \mathcal{G}_{0}^{\eta}(\varrho, \vartheta)$ and $x \in \mathcal{X}$,

$$
\Phi_{k}^{j}(h)(x):=\phi_{k}^{j}(x)\left[\int_{\mathcal{X}} \phi_{k}^{j}(z) d \mu(z)\right]^{-1} \int_{\mathcal{X}}[h(x)-h(z)] \phi_{k}^{j}(z) d \mu(z) .
$$

By [21, Lemma 4.9], we know that $\Phi_{k}^{j}$ is bounded on $\mathcal{G}_{0}^{\eta}(\varrho, \vartheta)$ with operator norm depending on $j$ and $k$. Therefore, it makes sense to define a distribution $b_{k}^{j}$ on $\mathcal{G}_{0}^{\eta}(\varrho, \vartheta)$ by setting, for any $h \in \mathcal{G}_{0}^{\eta}(\varrho, \vartheta)$,

$$
\left\langle b_{k}^{j}, h\right\rangle:=\left\langle f, \Phi_{k}^{j}(h)\right\rangle .
$$

In what follows, for any $j \in \mathbf{Z}$ and $k \in I_{j}$, let $x_{k}^{j} \in \Omega^{j}$ and $r_{k}^{j} \in(0, \infty)$ be defined as in [27, Propositions 4.4 and 4.5] with the open set $\Omega$ therein replaced by $\Omega^{j}$ as in (5.5). The following estimate of $\left(b_{k}^{j}\right)^{*}$ is taken from [40, Lemma 3.7].

Proposition 5.7. For any $j \in \mathbf{Z}$ and $k \in I_{j}$, let $b_{k}^{j}$ be defined by (5.6). Then there exists a positive constant $C$ such that, for any $j \in \mathbf{Z}, k \in I_{j}$ and $x \in \mathcal{X}$,

$\left(b_{k}^{j}\right)^{*}(x)$

$\leq C 2^{j} \frac{V\left(x_{k}^{j}, r_{k}^{j}\right)}{V\left(x_{k}^{j}, r_{k}^{j}\right)+V\left(x_{k}^{j}, x\right)}\left[\frac{r_{k}^{j}}{r_{k}^{j}+d\left(x_{k}^{j}, x\right)}\right]^{\varrho} \mathbf{1}_{\left(B\left(x_{k}^{j}, 16 A_{0} r_{k}^{j}\right)\right)^{\complement}}(x)+C f^{*} \mathbf{1}_{B\left(x_{k}^{j}, 16 A_{0} r_{k}^{j}\right)}(x)$.

Then we obtain the following Calderón-Zygmund decomposition, which is a generalization of the corresponding results on Hardy spaces $H^{p}(\mathcal{X})$ in $[27$, Proposition 4.9] [in this case, $\varphi$ is the same as in (4.2)].

Proposition 5.8. Let $\varphi$ be a growth function as in Definition 2.5. For any $f \in H^{*, \varphi}(\mathcal{X}) \subset\left(\mathcal{G}_{0}^{\eta}(\varrho, \vartheta)\right)^{\prime}$, with $\varrho$, $\vartheta$ as in (5.1), and, for any $j \in \mathbf{Z}$ and $k \in I_{j}$, letting $b_{k}^{j}$ be defined as in (5.6), then there exists a positive constant $C$ such that, for any $j \in \mathbf{Z}$ and $k \in I_{j}$,

$$
\int_{\mathcal{X}} \varphi\left(x,\left(b_{k}^{j}\right)^{*}(x)\right) d \mu(x) \leq C \int_{B\left(x_{k}^{j}, 16 A_{0}^{4} r_{k}^{j}\right)} \varphi\left(x, f^{*}(x)\right) d \mu(x)
$$

moreover, there exists $b^{j} \in H^{*, \varphi}(\mathcal{X})$ such that $b^{j}=\sum_{k \in I_{j}} b_{k}^{j}$ in $H^{*, \varphi}(\mathcal{X})$ and, for any $x \in \mathcal{X}$,

$$
\left(b^{j}\right)^{*}(x) \leq C 2^{j} \sum_{k \in I_{j}} \frac{V\left(x_{k}^{j}, r_{k}^{j}\right)}{V\left(x_{k}^{j}, r_{k}^{j}\right)+V\left(x_{k}^{j}, x\right)}\left[\frac{r_{k}^{j}}{r_{k}^{j}+d\left(x_{k}^{j}, x\right)}\right]^{\varrho}+C f^{*}(x) \mathbf{1}_{\Omega^{j}}(x) ;
$$



if $g^{j}:=f-b^{j}$ for any $j \in \mathbf{Z}$, then, for any $x \in \mathcal{X}$,

$$
\left(g^{j}\right)^{*}(x) \leq C 2^{j} \sum_{k \in I_{j}} \frac{V\left(x_{k}^{j}, r_{k}^{j}\right)}{V\left(x_{k}^{j}, r_{k}^{j}\right)+V\left(x_{k}^{j}, x\right)}\left[\frac{r_{k}^{j}}{r_{k}^{j}+d\left(x_{k}^{j}, x\right)}\right]^{\varrho}+C f^{*}(x) \mathbf{1}_{\left(\Omega^{j}\right) \mathrm{C}}(x) .
$$

Proof. Since (5.8) and (5.9) can be proved by the same arguments used in the proof of $[27,(4.5)$ and (4.6)], we only need to show (5.7) and that there exists $b^{j} \in H^{*, \varphi}(\mathcal{X})$ such that $b^{j}=\sum_{k \in I_{j}} b_{k}^{j}$ in $H^{*, \varphi}(\mathcal{X})$. Indeed, by Proposition 5.7, we write

$$
\begin{aligned}
& \int_{\mathcal{X}} \varphi\left(x,\left(b_{k}^{j}\right)^{*}(x)\right) d \mu(x) \\
& \lesssim \int_{\mathcal{X}} \varphi\left(x, 2^{j} \frac{V\left(x_{k}^{j}, r_{k}^{j}\right)}{V\left(x_{k}^{j}, r_{k}^{j}\right)+V\left(x_{k}^{j}, x\right)}\left[\frac{r_{k}^{j}}{r_{k}^{j}+d\left(x_{k}^{j}, x\right)}\right]^{\varrho} \mathbf{1}_{\left(B\left(x_{k}^{j}, 16 A_{0} r_{k}^{j}\right)\right)^{\mathrm{C}}}(x)\right) d \mu(x) \\
& \quad+\int_{\mathcal{X}} \varphi\left(x, f^{*}(x) \mathbf{1}_{B\left(x_{k}^{j}, 16 A_{0} r_{k}^{j}\right)}(x)\right) d \mu(x)=: \mathrm{I}+\mathrm{II} .
\end{aligned}
$$

By [27, (ii) and (v) of Proposition 4.4], we have, for any $j \in \mathbf{Z}$,

$$
\Omega^{j}=\bigcup_{k \in I_{j}} B\left(x_{k}^{j}, 16 A_{0} r_{k}^{j}\right) \text { and }\left\{B\left(x_{k}^{j}, 16 A_{0} r_{k}^{j}\right)\right\}_{k \in I_{j}} \text { is finitely overlapped }
$$

with the finite positive integer independent of $j$ and $k$. We immediately observe that

$$
\mathrm{II} \sim \int_{B\left(x_{k}^{j}, 16 A_{0}^{4} r_{k}^{j}\right)} \varphi\left(x, f^{*}(x)\right) d \mu(x) .
$$

For the estimation of I, choose $q>q(\varphi)$ such that $\omega\left(\frac{q}{p}-1\right)<\eta$ and $\varrho \in$ $\left(\omega\left[\frac{q}{p}-1\right], \eta\right)$. By $\varrho \in\left(\omega\left[\frac{q}{p}-1\right], \eta\right)$ and $f^{\star} \sim f^{*}$, we conclude that

$$
\begin{aligned}
\mathrm{I} & \lesssim \sum_{s=0}^{\infty} \int_{B\left(x_{k}^{j}, 2^{s+1} 16 A_{0} r_{k}^{j}\right) \backslash B\left(x_{k}^{j}, 2^{s} 16 A_{0} r_{k}^{j}\right)} \varphi\left(x, 2^{j} \frac{V\left(x_{k}^{j}, r_{k}^{j}\right)}{V\left(x_{k}^{j}, 2^{s} 16 A_{0}^{4} r_{k}^{j}\right)} 2^{-s \varrho}\right) d \mu(x) \\
& \lesssim \sum_{s=0}^{\infty} 2^{-s \varrho p}\left[\frac{V\left(x_{k}^{j}, r_{k}^{j}\right)}{V\left(x_{k}^{j}, 2^{s} 16 A_{0}^{4} r_{k}^{j}\right)}\right]^{p} \varphi\left(B\left(x_{k}^{j}, 2^{s+1} 16 A_{0} r_{k}^{j}\right), 2^{j}\right) \\
& \lesssim \sum_{s=0}^{\infty} 2^{-s \varrho p}\left[\frac{V\left(x_{k}^{j}, 2^{s} 16 A_{0}^{4} r_{k}^{j}\right)}{V\left(x_{k}^{j}, r_{k}^{j}\right)}\right]^{q-p} \varphi\left(B\left(x_{k}^{j}, 16 A_{0} r_{k}^{j}\right), 2^{j}\right) \\
& \lesssim \sum_{s=0}^{\infty} 2^{-s \varrho p} 2^{s \omega(q-p)} \varphi\left(B\left(x_{k}^{j}, 16 A_{0} r_{k}^{j}\right), 2^{j}\right) d \mu(x) \lesssim \int_{B\left(x_{k}^{j}, 16 A_{0}^{4} r_{k}^{j}\right)} \varphi\left(x, f^{*}(x)\right) d \mu(x) .
\end{aligned}
$$

Combining the estimates of I and II, we then complete the proof of (5.7).

Moreover, from Lemma 2.8(i), (5.7) and (5.10), we deduce that

$$
\begin{aligned}
& \int_{\mathcal{X}} \varphi\left(x,\left(\sum_{k \in I_{j}} b_{k}^{j}\right)^{*}(x)\right) d \mu(x) \\
& \lesssim \int_{\mathcal{X}} \varphi\left(x, \sum_{k \in I_{j}}\left(b_{k}^{j}\right)^{*}(x)\right) d \mu(x) \lesssim \sum_{k \in I_{j}} \int_{\mathcal{X}} \varphi\left(x,\left(b_{k}^{j}\right)^{*}(x)\right) d \mu(x)
\end{aligned}
$$




$$
\lesssim \sum_{k \in I_{j}} \int_{B\left(x_{k}^{j}, 16 A_{0}^{4} r_{k}^{j}\right)} \varphi\left(x, f^{*}(x)\right) d \mu(x) \lesssim \int_{\Omega^{j}} \varphi\left(x, f^{*}(x)\right) d \mu(x)<\infty,
$$

which, together with the completeness of $H^{*, \varphi}(\mathcal{X})$, further implies that there exist $\left\{b^{j}\right\}_{j \in I_{j}} \subset H^{*, \varphi}(\mathcal{X})$ such that $b^{j}=\sum_{k \in I_{j}} b_{k}^{j}$ in $H^{*, \varphi}(\mathcal{X})$. This finishes the proof of Proposition 5.8.

5.3. Atomic characterizations of $\boldsymbol{H}^{*, \varphi}(\mathcal{X})$. In this subsection, we prove that $H^{*, \varphi}(\mathcal{X}) \subset H_{\text {at }}^{\varphi, q}(\mathcal{X})$, namely, the atomic decomposition of $H^{*, \varphi}(\mathcal{X})$. To this end, we first need the following result on the density of $L_{\varphi(\cdot, 1)}^{q}(\mathcal{X}) \cap H^{*, \varphi}(\mathcal{X})$ in $H^{*, \varphi}(\mathcal{X})$, which is a variant of the corresponding Euclidean case in [35, Proposition 5.3].

Lemma 5.9. For any $q \in(q(\varphi), \infty), L_{\varphi(\cdot, 1)}^{q}(\mathcal{X}) \cap H^{*, \varphi}(\mathcal{X})$ is dense in $H^{*, \varphi}(\mathcal{X})$.

Proof. Let $f \in H^{*, \varphi}(\mathcal{X})$. By the Calderón-Zygmund decomposition of $f^{*}$ (see Proposition 5.8 with the same notation as therein), we conclude that, for any $j \in \mathbf{Z}$,

$$
f=g^{j}+b^{j}=g^{j}+\sum_{k \in I_{j}} b_{k}^{j} .
$$

Now, we show that $g^{j} \in L_{\varphi(\cdot, 1)}^{q}(\mathcal{X}) \cap H^{*, \varphi}(\mathcal{X})$.

Indeed, for any $j \in \mathbf{Z}, k \in I_{j}$ and any $x \in \mathcal{X}$,

$$
\begin{aligned}
& \frac{V\left(x_{k}^{j}, r_{k}^{j}\right)}{V\left(x_{k}^{j}, r_{k}^{j}\right)+V\left(x_{k}^{j}, x\right)}\left[\frac{r_{k}^{j}}{r_{k}^{j}+d\left(x_{k}^{j}, x\right)}\right]^{\varrho} \\
& \lesssim \frac{V\left(x_{k}^{j}, r_{k}^{j}\right)}{V\left(x_{k}^{j}, r_{k}^{j}+d\left(x_{k}^{j}, x\right)\right)}\left[\frac{V\left(x_{k}^{j}, r_{k}^{j}\right)}{V\left(x_{k}^{j}, r_{k}^{j}+d\left(x_{k}^{j}, x\right)\right)}\right]^{\frac{\rho}{\omega}} \sim\left[\frac{V\left(x_{k}^{j}, r_{k}^{j}\right)}{V\left(x_{k}^{j}, r_{k}^{j}+d\left(x_{k}^{j}, x\right)\right)}\right]^{1+\frac{\rho}{\omega}} \\
& \sim\left\{\frac{1}{V\left(x_{k}^{j}, r_{k}^{j}+d\left(x_{k}^{j}, x\right)\right)} \int_{B\left(x_{k}^{j}, r_{k}^{j}+d\left(x_{k}^{j}, x\right)\right)} \mathbf{1}_{B\left(x_{k}^{j}, r_{k}^{j}\right)}(y) d \mu(y)\right\}^{1+\frac{\rho}{\omega}} \\
& \lesssim\left[M\left(\mathbf{1}_{B\left(x_{k}^{j}, r_{k}^{j}\right)}\right)(x)\right]^{1+\frac{\varrho}{\omega}} .
\end{aligned}
$$

In what follows, let $s_{0}:=1+\frac{\varrho}{\omega}$. From this, (5.9) and the Fefferman-Stein vectorvalued maximal inequality (see, for instance, [22, Theorem 1.2]), we deduce that

$$
\begin{aligned}
& \int_{\mathcal{X}}\left[\left(g^{j}\right)^{*}(x)\right]^{q} \varphi(x, 1) d \mu(x) \\
& \lesssim \int_{\mathcal{X}} 2^{j q}\left\{\sum_{k \in I_{j}} \frac{V\left(x_{k}^{j}, r_{k}^{j}\right)}{V\left(x_{k}^{j}, r_{k}^{j}\right)+V\left(x_{k}^{j}, x\right)}\left[\frac{r_{k}^{j}}{r_{k}^{j}+d\left(x_{k}^{j}, x\right)}\right]^{\varrho}\right\}^{q} \varphi(x, 1) d \mu(x) \\
& \quad+\int_{\left(\Omega^{j}\right)^{\mathrm{C}}}\left[f^{*}(x)\right]^{q} \varphi(x, 1) d \mu(x) \\
& \lesssim 2^{j q} \int_{\mathcal{X}}\left\{\left[\sum_{k \in I_{j}}\left\{M\left(\mathbf{1}_{B\left(x_{k}^{j}, r_{k}^{j}\right)}\right)(x)\right\}^{s_{0}}\right]^{\frac{1}{s_{0}}}\right\}^{s_{0} q} \varphi(x, 1) d \mu(x) \\
& \quad+\int_{\left(\Omega^{j}\right)^{\complement}}\left[f^{*}(x)\right]^{q} \varphi(x, 1) d \mu(x)
\end{aligned}
$$




$$
\begin{aligned}
& \lesssim 2^{j q} \int_{\mathcal{X}}\left\{\left[\sum_{k \in I_{j}}\left\{\mathbf{1}_{B\left(x_{k}^{j}, r_{k}^{j}\right)}(x)\right\}^{s_{0}}\right]^{\frac{1}{s_{0}}}\right\}^{s_{0} q} \varphi(x, 1) d \mu(x)+\int_{\left(\Omega^{j}\right)^{\mathrm{C}}}\left[f^{*}(x)\right]^{q} \varphi(x, 1) d \mu(x) \\
& \lesssim 2^{j q} \int_{\Omega^{j}} \varphi(x, 1) d \mu(x)+\int_{\left(\Omega^{j}\right)^{\mathrm{C}}}\left[f^{*}(x)\right]^{q} \varphi(x, 1) d \mu(x) \\
& \lesssim 2^{j q} \int_{\Omega^{j}} \varphi\left(x, \frac{f^{*}(x)}{2^{j}}\right) d \mu(x) \\
& +\int_{\left(\Omega^{j}\right)^{\mathrm{C}}}\left[f^{*}(x)\right]^{q} \max \left\{\frac{1}{\left[f^{*}(x)\right]^{p}}, \frac{1}{f^{*}(x)}\right\} \varphi\left(x, f^{*}(x)\right) d \mu(x) \\
& \lesssim 2^{j q} \max \left\{\frac{1}{2^{j p}}, \frac{1}{2}\right\} \int_{\Omega^{j}} \varphi\left(x, f^{*}(x)\right) d \mu(x) \\
& +\max \left\{2^{j(q-p)}, 2^{j(q-1)}\right\} \int_{\left(\Omega^{j}\right)^{\mathrm{C}}} \varphi\left(x, f^{*}(x)\right) d \mu(x) \\
& \sim 2^{j q} \max \left\{\frac{1}{2^{j p}}, \frac{1}{2}\right\} \int_{\mathcal{X}} \varphi\left(x, f^{*}(x)\right) d \mu(x)<\infty .
\end{aligned}
$$

Moreover, by (5.11), we know that

$$
\begin{aligned}
\int_{\mathcal{X}} \varphi\left(x,\left(g^{j}-f\right)^{*}(x)\right) d \mu(x) & =\int_{\mathcal{X}} \varphi\left(x,\left(b^{j}\right)^{*}(x)\right) d \mu(x) \\
& \lesssim \int_{\Omega^{j}} \varphi\left(x, f^{*}(x)\right) d \mu(x) \rightarrow 0
\end{aligned}
$$

as $j \rightarrow \infty$. Therefore, by Lemma 2.8(iii), we finally obtain

$$
\lim _{j \rightarrow \infty}\left\|g^{j}-f\right\|_{H^{*, \varphi}(\mathcal{X})}=0
$$

which completes the proof of Lemma 5.9.

Now, we are ready to prove $H^{*, \varphi}(\mathcal{X}) \subset H_{\text {at }}^{\varphi, q}(\mathcal{X})$. In what follows, with all the same notation as in Subsection 5.2, for any $j \in \mathbf{Z}$ and $k \in I_{j}$, let

$$
m_{k}^{j}:=\frac{1}{\left\|\phi_{k}^{j}\right\|_{L^{1}(\mathcal{X})}} \int_{\mathcal{X}} f(y) \phi_{k}^{j}(y) d \mu(y) \quad \text { and } \quad b_{k}^{j}:=\left(f-m_{k}^{j}\right) \phi_{k}^{j} .
$$

For any $q \in[1, \infty]$, we also use the symbol $L_{\varphi(\cdot, 1)}^{q}(\mathcal{X})$ to denote the weighted Lebesgue space equipped with the following norm: for any $f \in L_{\varphi(\cdot, 1)}^{q}(\mathcal{X})$,

$$
\|f\|_{L_{\varphi(\cdot, 1)}^{q}(\mathcal{X})}:=\left\{\int_{\mathcal{X}}|f(x)|^{q} \varphi(x, 1) d \mu(x)\right\}^{1 / q}
$$

with the usual modification made when $q=\infty$.

Proof of Theorem 5.4, Part II. In this step, we show $H^{*, \varphi}(\mathcal{X}) \subset H_{\mathrm{at}}^{\varphi, q}(\mathcal{X})$. By Lemma 5.9 and a standard density argument (see, for instance, [59, pp. 32-33]), we reduce the proof of this step to showing that, for any $f \in L_{\varphi(\cdot, 1)}^{q}(\mathcal{X}) \cap H^{*, \varphi}(\mathcal{X})$, $f \in H_{\mathrm{at}}^{\varphi, q}(\mathcal{X})$ and

$$
\|f\|_{H_{\mathrm{at}}^{\varphi, q}(\mathcal{X})} \lesssim\|f\|_{H^{*, \varphi}(\mathcal{X})}
$$


Indeed, let $f \in L_{\varphi(\cdot, 1)}^{q}(\mathcal{X}) \cap H^{*, \varphi}(\mathcal{X})$. By the Calderón-Zygmund decomposition of $f^{*}$ (see Proposition 5.8 with the same notation as therein), we conclude that, for any $j \in \mathbf{Z}$,

$$
f=g^{j}+b^{j}=g^{j}+\sum_{k \in I_{j}} b_{k}^{j} .
$$

For any $j \in \mathbf{N}$, let $h^{j}:=g^{j+1}-g^{j}=b^{j}-b^{j+1}$. Then $f-\sum_{j=-m}^{m} h^{j}=b^{m+1}+g^{-m}$. From (5.12), it follows that

$$
\lim _{j \rightarrow \infty}\left\|g^{j}-f\right\|_{H^{*, \varphi}(\mathcal{X})}=0 .
$$

Therefore, $\left\|\left(b^{m+1}\right)^{*}\right\|_{L^{\varphi}(\mathcal{X})}=\left\|g^{m+1}-f\right\|_{H^{*, \varphi}(\mathcal{X})} \rightarrow 0$ as $m \rightarrow \infty$. Observe that, by [27, Lemma 4.11(iv)], $g^{j} \rightarrow 0$ uniformly as $j \rightarrow-\infty$. Thus,

$$
f=\sum_{j=-\infty}^{\infty}\left(g^{j+1}-g^{j}\right) \quad \text { in }\left(\mathcal{G}_{0}^{\eta}(\varrho, \vartheta)\right)^{\prime} .
$$

Besides, from the proof of $H^{*, p}(\mathcal{X}) \subset H_{\mathrm{at}}^{p, q}(\mathcal{X})$ in [27], we deduce that

$$
h^{j}=b^{j}-b^{j+1}=\sum_{k \in I_{j}}\left[b_{k}^{j}-\sum_{l \in I_{j+1}} b_{l}^{j+1} \phi_{k}^{j}-L_{k, l}^{j+1} \phi_{l}^{j+1}\right]=: \sum_{k \in I_{j}} h_{k}^{j}
$$

converges in $\left(\mathcal{G}_{0}^{\eta}(\varrho, \vartheta)\right)^{\prime}$ and almost everywhere on $\mathcal{X}$,

$$
\operatorname{supp} h_{k}^{j} \subset B_{k}^{j}:=B\left(x_{k}^{j}, 16 A_{0}^{4} r_{k}^{j}\right) \quad \text { and } \quad\left\|h_{k}^{j}\right\|_{L^{\infty}(\mathcal{X})} \leq C_{4} 2^{j} \text {, }
$$

where $C_{4}$ is some fixed positive constant. Let

$$
\lambda_{k}^{j}:=C_{4} 2^{j}\left\|\mathbf{1}_{B_{k}^{j}}\right\|_{L^{\varphi}(\mathcal{X})} \quad \text { and } \quad a_{k}^{j}:=\frac{h_{k}^{j}}{\lambda_{k}^{j}} .
$$

Then $a_{k}^{j}$ is a $(\varphi, \infty)$-atom with $\operatorname{supp} a_{k}^{j} \subset B_{k}^{j}$ and

$$
f=\sum_{j=-\infty}^{\infty} \sum_{k \in I_{j}} h_{k}^{j} \quad \text { in }\left(\mathcal{G}_{0}^{\eta}(\varrho, \vartheta)\right)^{\prime} .
$$

Furthermore, by (5.14) and Lemma 2.8(ii), we obtain

$$
\begin{aligned}
& \sum_{j \in \mathbf{Z}} \sum_{k \in I_{j}} \varphi\left(B_{k}^{j}, \frac{\left\|h_{k}^{j}\right\|_{L^{\infty}(\mathcal{X})}}{\|f\|_{H^{*, \varphi}(\mathcal{X})}}\right) \\
& \lesssim \sum_{j \in \mathbf{Z}} \varphi\left(\Omega^{j}, \frac{2^{j}}{\|f\|_{H^{*, \varphi}(\mathcal{X})}}\right) \sim \sum_{j \in \mathbf{Z}} \sum_{\ell=j}^{\infty} \varphi\left(\Omega^{\ell} \backslash \Omega^{\ell+1}, \frac{2^{j}}{\|f\|_{H^{*, \varphi}(\mathcal{X})}}\right) \\
& \lesssim \sum_{\ell \in \mathbf{Z}} \sum_{j=-\infty}^{\ell} 2^{(j-\ell) p} \varphi\left(\Omega^{\ell} \backslash \Omega^{\ell+1}, \frac{2^{\ell}}{\|f\|_{H^{*, \varphi}(\mathcal{X})}}\right) \lesssim \sum_{\ell \in \mathbf{Z}} \varphi\left(\Omega^{\ell} \backslash \Omega^{\ell+1}, \frac{f^{*}}{\|f\|_{H^{*, \varphi}(\mathcal{X})}}\right) \\
& \sim \int_{\mathcal{X}} \varphi\left(x, \frac{f^{*}(x)}{\|f\|_{H^{*, \varphi}(\mathcal{X})}}\right) d \mu(x) \sim 1 .
\end{aligned}
$$

Consequently, $\|f\|_{H_{\mathrm{at}}^{\varphi, q}(\mathcal{X})} \lesssim\|f\|_{H_{\mathrm{at}}^{\varphi, \infty}(\mathcal{X})} \lesssim\|f\|_{H^{*, \varphi}(\mathcal{X})}$, which completes the proof of $H^{*, \varphi}(\mathcal{X}) \subset H_{\mathrm{at}}^{\varphi, q}(\mathcal{X})$ and hence of Part II of the proof of Theorem 5.4. So far, the proof of Theorem 5.4 is then completed. 


\section{Littlewood-Paley characterizations of $\boldsymbol{H}_{\mathrm{at}}^{\varphi}(\mathcal{X})$}

In this section, we investigate the Littlewood-Paley characterizations of $H_{\mathrm{at}}^{\varphi}(\mathcal{X})$. We first recall the notions of the Lusin area function, the Littlewood-Paley $g$-function and the Littlewood-Paley $g_{\lambda}^{*}$-function from [27, Section 5]. Throughout the whole section, we assume that $q(\varphi)<\frac{p(\eta+\omega)}{\omega}$, which is sharp in the sense that, if $\varphi(x, t)=t^{p}$ for any $(x, t) \in \mathcal{X} \times[0, \infty)$, then $q(\varphi)=1$ and hence $q(\varphi)<\frac{p(\eta+\omega)}{\omega}$ if and only if $p>\frac{\omega}{\eta+\omega}$, which returns to the classical case.

Definition 6.1. Let $\varphi$ be a growth function as in Definition 2.5 and $\varrho, \vartheta$ as in (5.1), and let $f \in\left(\dot{\mathcal{G}}_{0}^{\eta}(\varrho, \vartheta)\right)^{\prime}$ and $\left\{Q_{k}\right\}_{k \in \mathbf{Z}}$ be an exp-ATI.

(i) For any $\theta \in(0, \infty)$, the Lusin area function of $f$ with aperture $\theta, \mathcal{S}_{\theta}(f)$, is defined by setting, for any $x \in \mathcal{X}$,

$$
\mathcal{S}_{\theta}(f)(x):=\left[\sum_{k \in \mathbf{Z}} \int_{B\left(x, \theta \delta^{k}\right)}\left|Q_{k} f(y)\right|^{2} \frac{d \mu(y)}{V_{\theta \delta^{k}}(x)}\right]^{\frac{1}{2}} .
$$

Particularly, we simply write $\mathcal{S}:=\mathcal{S}_{1}$.

(ii) The Littlewood-Paley $g$-function of $f, g(f)$, is defined by setting, for any $x \in \mathcal{X}$,

$$
g(f)(x):=\left[\sum_{k \in \mathbf{Z}}\left|Q_{k} f(x)\right|^{2}\right]^{\frac{1}{2}} .
$$

(iii) Let $\lambda \in(0, \infty)$. The Littlewood-Paley $g_{\lambda}^{*}$-function of $f, g_{\lambda}^{*}(f)$, is defined by setting, for any $x \in \mathcal{X}$,

$$
g_{\lambda}^{*}(f)(x):=\left\{\sum_{k \in \mathbf{Z}} \int_{\mathcal{X}}\left|Q_{k} f(y)\right|^{2}\left[\frac{\delta^{k}}{\delta^{k}+d(x, y)}\right]^{\lambda} \frac{d \mu(y)}{V_{\delta^{k}}(x)+V_{\delta^{k}}(y)}\right\}^{\frac{1}{2}} .
$$

Then we introduce the notion of Musielak-Orlicz Hardy spaces via Lusin area functions as follows.

Definition 6.2. Let $\varphi$ be a growth function as in Definition 2.5 and $\varrho, \vartheta$ as in (5.1). The Musielak-Orlicz Hardy space $H^{\varphi}(\mathcal{X})$, related to the Lusin area function, is defined by setting

$$
H^{\varphi}(\mathcal{X}):=\left\{f \in\left(\stackrel{\circ}{\mathcal{G}}_{0}^{\eta}(\varrho, \vartheta)\right)^{\prime}:\|\mathcal{S}(f)\|_{L^{\varphi}(\mathcal{X})}<\infty\right\}
$$

and, moreover, for any $f \in H^{\varphi}(\mathcal{X})$, let

$$
\|f\|_{H^{\varphi}(\mathcal{X})}:=\|\mathcal{S}(f)\|_{L^{\varphi}(\mathcal{X})} .
$$

6.1. Independence of exp-ATIs. In this section, we prove that $H^{\varphi}(\mathcal{X})$ is independent of the choices of exp-ATIs. Let $\mathcal{E}:=\left\{E_{k}\right\}_{k \in \mathbf{Z}}$ and $\mathcal{Q}:=\left\{Q_{k}\right\}_{k \in \mathbf{Z}}$ be two exp-ATIs, we then let $\mathcal{S}_{\mathcal{E}}$ and $\mathcal{S}_{\mathcal{Q}}$ be the Lusin area functions associated with $\mathcal{E}$ and $\mathcal{Q}$, respectively.

Theorem 6.3. Let $\varphi$ be a growth function as in Definition 2.5, $\varrho, \vartheta \in\left(\omega\left[\frac{q(\varphi)}{p}-\right.\right.$ 1], $\eta), \mathcal{E}:=\left\{E_{k}\right\}_{k \in \mathbf{Z}}$ and $\mathcal{Q}:=\left\{Q_{k}\right\}_{k \in \mathbf{Z}}$ be two exp-ATIs. Then there exists a positive constant $C$ such that, for any $f \in\left(\stackrel{\mathcal{G}}{0}_{0}^{\eta}(\varrho, \vartheta)\right)^{\prime}$,

$$
C^{-1}\left\|\mathcal{S}_{\mathcal{Q}}(f)\right\|_{L^{\varphi}(\mathcal{X})} \leq\left\|\mathcal{S}_{\mathcal{E}}(f)\right\|_{L^{\varphi}(\mathcal{X})} \leq C\left\|\mathcal{S}_{\mathcal{Q}}(f)\right\|_{L^{\varphi}(\mathcal{X})} .
$$


In order to prove Theorem 6.3, we need to first establish the Fefferman-Stein vector-valued maximal inequality for $L^{\varphi}(\mathcal{X})$. We first state the following vectorvalued interpolation theorem of Musielak-Orlicz type, which is similar to the corresponding Euclidean case in [41, Theorem 2.9]; the details are omitted.

Recall that the space $L^{\varphi}\left(\ell^{r}, \mathcal{X}\right)$ is defined to be the set of all measurable functions $\left\{f_{j}\right\}_{j}$ such that

$$
\left[\sum_{j}\left|f_{j}\right|^{r}\right]^{1 / r} \in L^{\varphi}(\mathcal{X})
$$

equipped with the (quasi-)norm

$$
\left\|\left\{f_{j}\right\}_{j}\right\|_{L^{\varphi}\left(\ell^{r}, \mathcal{X}\right)}:=\left\|\left[\sum_{j}\left|f_{j}\right|^{r}\right]^{1 / r}\right\|_{L^{\varphi}(\mathcal{X})} .
$$

Theorem 6.4. Let $p_{1}, p_{2} \in(0, \infty), p_{1}<p_{2}, r \in[1, \infty)$ and $\varphi$ be a MusielakOrlicz function of uniformly lower type $p_{\varphi}^{-}$and of uniformly upper type $p_{\varphi}^{+}$. Suppose that $0<p_{1}<p_{\varphi}^{-}<p_{\varphi}^{+}<p_{2}<\infty$ and $T$ is a linear operator defined on $L_{\varphi(\cdot, 1)}^{p_{1}}(\mathcal{X})+$ $L_{\varphi(\cdot, 1)}^{p_{2}}(\mathcal{X})$ satisfying that there exist positive constants $C_{1}$ and $C_{2}$ such that, for any $i \in\{1,2\}$ and any sequence $\left\{f_{j}\right\}_{j} \in L^{p_{i}}\left(\ell^{r}, \mathcal{X}\right), \alpha \in(0, \infty)$ and $t \in(0, \infty)$,

$$
\begin{aligned}
& \varphi\left(\left\{x \in \mathcal{X}:\left[\sum_{j}\left|T\left(f_{j}\right)(x)\right|^{r}\right]^{\frac{1}{r}}>\alpha\right\}, t\right) \\
& \leq C_{i} \alpha^{-p_{i}} \int_{\mathcal{X}}\left[\sum_{j}\left|f_{j}(x)\right|^{r}\right]^{\frac{p_{i}}{r}} \varphi(x, t) d \mu(x) .
\end{aligned}
$$

Then there exists a positive constant $C$ such that, for any sequence $\left\{f_{j}\right\}_{j} \in L^{\varphi}\left(\ell^{r}, \mathcal{X}\right)$,

$$
\int_{\mathcal{X}} \varphi\left(x,\left[\sum_{j}\left|T\left(f_{j}\right)(x)\right|^{r}\right]^{\frac{1}{r}}\right) d \mu(x) \leq C \int_{\mathcal{X}} \varphi\left(x,\left[\sum_{j}\left|f_{j}(x)\right|^{r}\right]^{\frac{1}{r}}\right) d \mu(x) .
$$

By the Calderón-Zygmund decomposition (see Lemma 3.3) and some arguments similar to those used in the proof of the Euclidean case in [1, Theorme 3.1], we obtain the corresponding result on $\mathcal{X}$ and omit the details here.

Theorem 6.5. Let $r \in(1, \infty]$.

(i) If $p \in[1, \infty)$, then there exists a positive constant $C_{(r, p)}$, depending on $r$ and $p$, such that $w \in A_{p}(\mathcal{X})$ if and only if

$$
\omega\left(\left\{x \in \mathcal{X}:\left[\sum_{j}\left\{M\left(f_{j}\right)(x)\right\}^{r}\right]^{\frac{1}{r}}>\alpha\right\}\right) \leq C_{(r, p)} t^{-p} \int_{\mathcal{X}}\left[\sum_{j}\left|f_{j}(x)\right|^{r}\right]^{\frac{p}{r}} \omega(x) d \mu(x) .
$$

(ii) If $p \in(1, \infty)$, then there exists a positive constant $C_{(r, p)}$, depending on $r$ and $p$, such that $w \in A_{p}(\mathcal{X})$ if and only if

$$
\int_{\mathcal{X}}\left[\sum_{j}\left\{M\left(f_{j}\right)(x)\right\}^{r}\right]^{\frac{p}{r}} \omega(x) d \mu(x) \leq C_{(r, p)} \int_{\mathcal{X}}\left[\sum_{j}\left|f_{j}(x)\right|^{r}\right]^{\frac{p}{r}} \omega(x) d \mu(x) .
$$


For more study on Muckenhoupt weights on spaces of homogeneous type, we refer the reader to [58, Chapter 1$]$.

Combining Theorems 6.4 and 6.5, we obtain the following vector-valued Fefferman-Stein maximal inequality on spaces of homogeneous type, which is a generalization of the corresponding Euclidean case in [41, Theorem 2.10]; the details are omitted.

Theorem 6.6. Let $r \in(1, \infty], \varphi$ be a Musielak-Orlicz function of uniformly lower type $p_{\varphi}^{-}$and of uniformly upper type $p_{\varphi}^{+}$, and $\varphi \in \mathbf{A}_{q}(\mathcal{X})$. If $q(\varphi)<p_{\varphi}^{-}<p_{\varphi}^{+}<$ $\infty$, then there exists a positive constant $C$ such that, for any $\left\{f_{j}\right\}_{j \in \mathbf{Z}} \in L^{\varphi}\left(\ell^{r}, \mathcal{X}\right)$,

$$
\int_{\mathcal{X}} \varphi\left(x,\left[\sum_{j}\left\{M\left(f_{j}\right)(x)\right\}^{r}\right]^{\frac{1}{r}}\right) d \mu(x) \leq C \int_{\mathcal{X}} \varphi\left(x,\left[\sum_{j}\left|f_{j}(x)\right|^{r}\right]^{\frac{1}{r}}\right) d \mu(x) .
$$

Now, we are ready to show Theorem 6.3.

Proof of Theorem 6.3. By symmetry, we only need to show $\left\|\mathcal{S}_{\mathcal{E}}(f)\right\|_{L^{\varphi}(\mathcal{X})} \lesssim$ $\left\|\mathcal{S}_{\mathcal{Q}}(f)\right\|_{L^{\varphi}(\mathcal{X})}$. For any $k \in \mathbf{Z}, f \in\left(\dot{\mathcal{G}}_{0}^{\eta}(\varrho, \vartheta)\right)^{\prime}$ with $\varrho$ and $\vartheta$ as in this theorem, and $x \in \mathcal{X}$, let

$$
m_{k}(f)(x):=\left[\frac{1}{V_{\delta^{k}}(x)} \int_{B\left(x, \delta^{k}\right)}\left|Q_{k} f(y)\right|^{2} d \mu(y)\right]^{\frac{1}{2}} .
$$

Choose $\widetilde{\varrho}$ and $r$ such that $r \in(\omega /[\omega+\widetilde{\varrho}], p / q(\varphi))$. Then, by the proof of $[27$, Theorem 5.1], we conclude that, for any $x \in \mathcal{X}$,

$$
\left[\mathcal{S}_{\mathcal{E}}(f)(x)\right]^{2} \lesssim \sum_{k \in \mathbf{Z}}\left\{\mathcal{M}\left(\left[m_{k}(f)\right]^{r}\right)(x)\right\}^{\frac{2}{r}}
$$

which, combined with Theorem 6.6, further implies that

$$
\begin{aligned}
\left\|\mathcal{S}_{\mathcal{E}}(f)\right\|_{L^{\varphi}(\mathcal{X})} & \lesssim\left\|\left[\sum_{k \in \mathbf{Z}}\left\{\mathcal{M}\left(\left[m_{k}(f)\right]^{r}\right)\right\}^{\frac{2}{r}}\right]^{\frac{r}{2}}\right\|_{L^{\tilde{\varphi}}(\mathcal{X})}^{\frac{1}{r}} \\
& \lesssim\left\|\left\{\sum_{k \in \mathbf{Z}}\left[m_{k}(f)\right]^{2}\right\}^{\frac{1}{2}}\right\|_{L^{\varphi}(\mathcal{X})} \sim\left\|\mathcal{S}_{\mathcal{Q}}(f)\right\|_{L^{\varphi}(\mathcal{X})},
\end{aligned}
$$

where, for any $x \in \mathcal{X}$ and $t \in(0, \infty), \widetilde{\varphi}(x, t):=\varphi\left(x, t^{1 / r}\right)$ is of uniformly lower type $p / r \in(q(\varphi), \infty)$. This finishes the proof of Theorem 6.3.

6.2. Molecular characterizations of $\boldsymbol{H}_{\mathrm{at}}^{\varphi}(\mathcal{X})$. In this subsection, we establish the molecular characterization of $H_{\mathrm{at}}^{\varphi}(\mathcal{X})$. To this end, we first introduce the following notion of molecular Musielak-Orlicz Hardy spaces on $\mathcal{X}$, which is a generalization of the corresponding Euclidean case in [30] (see also [59]).

Definition 6.7. Let $\varphi$ be a growth function as in Definition $2.5, q \in(1, \infty)$, $s \in \mathbf{Z}_{+}$and $\epsilon \in(0, \infty)$. A measurable function $\alpha$ is called a $(\varphi, q, \epsilon)$-molecule, related to a ball $B$, if the following two conditions hold true:

(i) for any $j \in \mathbf{Z}_{+}$,

$$
\|\alpha\|_{L^{q}\left(U_{j}(B)\right)} \leq \delta^{j \epsilon}\left[\mu\left(\delta^{-j} B\right)\right]^{1 / q}\left\|\mathbf{1}_{B}\right\|_{L^{\varphi}(\mathcal{X})}^{-1},
$$

where $U_{0}(B):=B$ and $U_{j}(B):=\delta^{-j} B \backslash \delta^{-j+1} B$ for any $j \in \mathbf{N}$;

(ii) $\int_{\mathcal{X}} \alpha(x) d \mu(x)=0$. 
The molecular Musielak-Orlicz Hardy space $H_{\text {mol }}^{\varphi, q, \epsilon}(\mathcal{X})$ is defined to be the set of all $f \in\left(\mathcal{G}_{0}^{\eta}(\varrho, \vartheta)\right)^{\prime}$ with $\varrho, \vartheta \in\left(\omega\left[\frac{q(\varphi)}{p}-1\right], \eta\right)$ satisfying that there exist a sequence of $(\varphi, q, \epsilon)$-molecules, $\left\{\alpha_{j}\right\}_{j \in \mathbf{N}}$, related, respectively, to balls $\left\{B_{j}\right\}_{j \in \mathbf{N}}$, and $\left\{\lambda_{j}\right\}_{j \in \mathbf{N}} \subset \mathbf{C}$ such that

$$
\sum_{j=1}^{\infty} \varphi\left(B_{j}, \frac{\left|\lambda_{j}\right|}{\left\|\mathbf{1}_{B_{j}}\right\|_{L^{\varphi}(\mathcal{X})}}\right)<\infty
$$

and $f=\sum_{j=1}^{\infty} \lambda_{j} \alpha_{j}$ in $\left(\mathcal{G}_{0}^{\eta}(\varrho, \vartheta)\right)^{\prime}$. Moreover, let

$$
\Lambda_{q}\left(\left\{\lambda_{j} \alpha_{j}\right\}_{j=1}^{\infty}\right):=\inf \left\{\lambda \in(0, \infty): \sum_{j=1}^{\infty} \varphi\left(B_{j}, \frac{\left|\lambda_{j}\right|}{\lambda\left\|\mathbf{1}_{B_{j}}\right\|_{L^{\varphi}(\mathcal{X})}}\right) \leq 1\right\}
$$

and then define

$$
\|f\|_{H_{\mathrm{mol}}^{\varphi, q, \epsilon}(\mathcal{X})}:=\inf \left\{\Lambda_{q}\left(\left\{\lambda_{j} \alpha_{j}\right\}_{j=1}^{\infty}\right): f=\sum_{j=1}^{\infty} \lambda_{j} \alpha_{j} \quad \text { in } \quad\left(\dot{\mathcal{G}}_{0}^{\eta}(\varrho, \vartheta)\right)^{\prime}\right\}
$$

where the last infimum is taken over all decompositions of $f$ as above.

The following molecular characterization of $H_{\mathrm{at}}^{\varphi}(\mathcal{X})$ is a generalization of the Euclidean case in [30, Theorem 4.13] to any space of homogeneous type (see also [59, Theorem 3.2.10]).

Theorem 6.8. Let $\varphi$ be a growth function as in Definition $2.5, \epsilon \in(\max \{\omega$, $\left.\left.\omega \frac{q(\varphi)}{i(\varphi)}\right\}, \infty\right), q \in\left(q(\varphi)[r(\varphi)]^{\prime}, \infty\right)$ and $m(\varphi) \leq 0$, where $q(\varphi), i(\varphi)$ and $m(\varphi)$ are, respectively, as in (3.16), (2.1) and (3.15), and

$$
r(\varphi):=\sup \left\{q \in(1, \infty]: \varphi \in \mathbf{R H}_{q}(\mathcal{X})\right\}
$$

where $\frac{1}{r(\varphi)}+\frac{1}{[r(\varphi)]^{\prime}}=1$. Then $H_{\text {mol }}^{\varphi, q, \epsilon}(\mathcal{X})=H_{\text {at }}^{\varphi}(\mathcal{X})$ with equivalent quasi-norms.

Proof. Let $\varphi$ be a growth function, $\epsilon \in(\max \{\omega, \omega q(\varphi) / i(\varphi)\}, \infty), q \in\left(q(\varphi)[r(\varphi)]^{\prime}\right.$, $\infty)$ and $m(\varphi) \leq 0$. Then $(\varphi, q)$ is admissible as in Definition 5.2.

First, we easily observe that any $(\varphi, \infty)$-atom is a $(\varphi, q, \epsilon)$-molecule. Thus, by Remark 5.5, we have

$$
H_{\mathrm{at}}^{\varphi}(\mathcal{X})=H_{\mathrm{at}}^{\varphi, \infty}(\mathcal{X}) \subset H_{\mathrm{mol}}^{\varphi, q, \epsilon}(\mathcal{X})
$$

and, for any $f \in H_{\text {at }}^{\varphi}(\mathcal{X})$,

$$
\|f\|_{H_{\mathrm{mol}}^{\varphi, q, \epsilon}(\mathcal{X})} \lesssim\|f\|_{H_{\mathrm{at}}^{\varphi, \infty}(\mathcal{X})} \sim\|f\|_{H_{\mathrm{at}}^{\varphi}(\mathcal{X})} .
$$

Now, we show that $H_{\text {mol }}^{\varphi, q, \epsilon}(\mathcal{X}) \subset H_{\text {at }}^{\varphi, q}(\mathcal{X})$. Indeed, let $m$ be any fixed $(\varphi, q, \epsilon)$ molecule related to a ball $B:=B\left(x_{B}, r_{B}\right)$ with $x_{B} \in \mathcal{X}$ and $r_{B} \in(0, \infty)$. It suffices to prove that $m$ is a countable linear combination of $(\varphi, \widetilde{q})$-atoms for some $\widetilde{q} \in(q(\varphi), \infty)$ which is determined later, and $(\varphi, \infty)$-atoms. let

To show this, write $R_{0}:=B$ and, for any $k \in \mathbf{N}, R_{k}:=\delta^{-k} B \backslash \delta^{-k+1} B$, and then

$$
M_{k}:=m \mathbf{1}_{R_{k}}-\frac{\mathbf{1}_{\delta^{-k} B}}{\mu\left(\delta^{-k} B\right)} \int_{\mathcal{X}} m(y) \mathbf{1}_{R_{k}}(y) d \mu(y)
$$

and

$$
m_{k}:=\frac{\mathbf{1}_{\delta^{-k} B}}{\mu\left(\delta^{-k} B\right)} \int_{\mathcal{X}} m(y) \mathbf{1}_{R_{k}}(y) d \mu(x)
$$


Then

$$
m=\sum_{k=0}^{\infty} M_{k}+\sum_{k=0}^{\infty} m_{k} .
$$

We first deal with $\sum_{k=0}^{\infty} M_{k}$. Fix $k \in \mathbf{Z}_{+}$. Now, we claim that $M_{k}$ is a multiple of a $(\varphi, \widetilde{q})$-atom for some $\widetilde{q} \in(q(\varphi), \infty)$ which is determined later. Indeed, it is obvious that $\int_{\mathcal{X}} M_{k}(y) d \mu(y)=0$ and $\operatorname{supp} M_{k} \subset \delta^{-k} B$. By $\epsilon>\omega>\frac{\omega}{q}$ and the fact that

$$
\left\|M_{k}\right\|_{L^{q}(\mathcal{X})} \lesssim\left\|m \mathbf{1}_{R_{k}}\right\|_{L^{q}(\mathcal{X})} \lesssim \delta^{k \epsilon}\left[\mu\left(\delta^{-k} B\right)\right]^{1 / q}\left\|\mathbf{1}_{B}\right\|_{L^{\varphi}(\mathcal{X})}^{-1}
$$

we conclude that

$$
\begin{aligned}
\left\|\sum_{k=0}^{\infty} M_{k}\right\|_{L^{q}(\mathcal{X})} & \leq \sum_{k=0}^{\infty}\left\|M_{k}\right\|_{L^{q}(\mathcal{X})} \lesssim \sum_{k=0}^{\infty} \delta^{k \epsilon}\left[\mu\left(\delta^{-k} B\right)\right]^{1 / q}\left\|\mathbf{1}_{B}\right\|_{L^{\varphi}(\mathcal{X})}^{-1} \\
& \lesssim \sum_{k=0}^{\infty} \delta^{k \epsilon} \delta^{-k \frac{\omega}{q}}[\mu(B)]^{1 / q}\left\|\mathbf{1}_{B}\right\|_{L^{\varphi}(\mathcal{X})}^{-1} \sim \sum_{k=0}^{\infty} \delta^{k\left(\epsilon-\frac{\omega}{q}\right)}[\mu(B)]^{1 / q}\left\|\mathbf{1}_{B}\right\|_{L^{\varphi}(\mathcal{X})}^{-1} \\
& \lesssim[\mu(B)]^{1 / q}\left\|\mathbf{1}_{B}\right\|_{L^{\varphi}(\mathcal{X})}^{-1}<\infty
\end{aligned}
$$

which further implies that $\sum_{k=0}^{\infty} M_{k}$ converges in $L^{q}(\mathcal{X})$ and hence in $\left(\mathcal{G}_{0}^{\eta}(\varrho, \vartheta)\right)^{\prime}$ with $\varrho, \vartheta \in\left(\omega\left[\frac{q(\varphi)}{p}-1\right], \eta\right)$.

Since $q>q(\varphi)[r(\varphi)]^{\prime}$, it follows that there exists $\widetilde{q} \in(q(\varphi), \infty)$ such that $q>$ $\widetilde{q}[r(\varphi)]^{\prime}$, which implies that $\varphi \in \mathbf{R H}_{\left(\frac{q}{q}\right)^{\prime}}(\mathcal{X})$. By this, the Hölder inequality and (6.11), we find that, for any $t \in(0, \infty)$,

$$
\begin{aligned}
& \left\{\frac{1}{\varphi\left(\delta^{-k} B, t\right)} \int_{\delta^{-k} B}\left[M_{k}(y)\right]^{\widetilde{q}} \varphi(y, t) d \mu(y)\right\}^{\frac{1}{\tilde{q}}} \\
& \leq \frac{1}{\left[\varphi\left(\delta^{-k} B, t\right)\right]^{\frac{1}{\tilde{q}}}}\left\|M_{k}\right\|_{L^{q}(\mathcal{X})}\left\{\int_{\delta^{-k} B}[\varphi(y, t)]^{\left(\frac{q}{q}\right)^{\prime}} d \mu(y)\right\}^{\frac{1}{\tilde{q}\left(\frac{q}{q}\right)^{\prime}}} \\
& \lesssim \frac{1}{\left[\varphi\left(\delta^{-k} B, t\right)\right]^{\frac{1}{\tilde{q}}}} \delta^{k \epsilon}\left[\mu\left(\delta^{-k} B\right)\right]^{1 / q}\left\|\mathbf{1}_{B}\right\|_{L^{\varphi}(\mathcal{X})}^{-1}\left[\mu\left(\delta^{-k} B\right)\right]^{\left(\frac{1}{\left(\frac{q}{q}\right)^{\prime}}-1\right) \frac{1}{\tilde{q}}}\left[\varphi\left(\delta^{-k} B, t\right)\right]^{\frac{1}{\tilde{q}}} \\
& \lesssim \delta^{k \epsilon}\left\|\mathbf{1}_{B}\right\|_{L^{\varphi}(\mathcal{X})}^{-1}=: \widetilde{C}_{2} \delta^{k \epsilon}\left\|\mathbf{1}_{B}\right\|_{L^{\varphi}(\mathcal{X})}^{-1},
\end{aligned}
$$

where $\widetilde{C}_{2}$ is a fixed positive constant. Thus,

$$
\left\|M_{k}\right\|_{L_{\varphi}^{\widetilde{q}(\mathcal{X})}} \leq \widetilde{C}_{2} \delta^{k \epsilon}\left\|\mathbf{1}_{B}\right\|_{L^{\varphi}(\mathcal{X})}^{-1}
$$

which further implies that $\frac{\delta^{-k \epsilon}}{\widetilde{C}_{2}} M_{k} \frac{\left\|\mathbf{1}_{B}\right\|}{\left\|\mathbf{1}_{\delta^{-} k_{B}}\right\|_{L^{\varphi}}(\mathcal{X})}$ is a $(\varphi, \widetilde{q})$-atom. This shows the above claim.

Next, we consider $\sum_{k=0}^{\infty} m_{k}$. For a fixed $k \in \mathbf{Z}_{+}$, let

$$
\mathbf{1}_{k}:=\frac{\mathbf{1}_{\delta^{-k} B}}{\mu\left(\delta^{-k} B\right)} \quad \text { and } \quad \tilde{m}_{k}:=\int_{\mathcal{X}} m(y) \mathbf{1}_{R_{k}}(y) d \mu(y)
$$


For any $k \in \mathbf{Z}_{+}$, let $N_{k}:=\sum_{j=k}^{\infty} \widetilde{m}_{j}$. We observe that $\int_{\mathcal{X}} m(y) d \mu(y)=0$ and hence $N_{0}=0$. Therefore,

$$
\begin{aligned}
\sum_{k=0}^{\infty} m_{k} & =\sum_{k=0}^{\infty} \mathbf{1}_{k} \widetilde{m}_{k}=\sum_{k=0}^{\infty} \mathbf{1}_{k}\left(N_{k}-N_{k+1}\right) \\
& =\sum_{k=0}^{\infty}\left(\mathbf{1}_{k}-\mathbf{1}_{k-1}\right) N_{k}+N_{0}=\sum_{k=0}^{\infty} \sum_{j=k}^{\infty}\left(\mathbf{1}_{k}-\mathbf{1}_{k-1}\right) \tilde{m}_{j}
\end{aligned}
$$

For any $k \in \mathbf{Z}_{+}$and $j \in \mathbf{Z}_{+} \cap[k, \infty)$, let $b_{k, j}:=\left(\mathbf{1}_{k}-\mathbf{1}_{k-1}\right) \tilde{m}_{j}$. We further prove that $b_{k, j}$ is a multiple of a $(\varphi, \infty)$-atom. Indeed, it is easy to find that $\operatorname{supp} b_{k, j} \subset \delta^{-k} B$ and $\int_{\mathcal{X}} b_{k, j}(y) d \mu(y)=0$. Also, by the Hölder inequality and (1.4), we have

$$
\begin{aligned}
\left\|b_{k, j}\right\|_{L^{\infty}(\mathcal{X})} & \lesssim \frac{1}{\mu\left(\delta^{-k} B\right)}\left\|m \mathbf{1}_{R_{j}}\right\|_{L^{1}(\mathcal{X})} \lesssim \frac{1}{\mu\left(\delta^{-k} B\right)}\left\|m \mathbf{1}_{R_{j}}\right\|_{L^{q}(\mathcal{X})}\left[\mu\left(\delta^{-j} B\right)\right]^{\frac{1}{q^{\prime}}} \\
& \lesssim \frac{1}{\mu\left(\delta^{-k} B\right)} \delta^{j \epsilon}\left\|\mathbf{1}_{B}\right\|_{L^{\varphi}(\mathcal{X})}^{-1}\left[\mu\left(\delta^{-j} B\right)\right]^{\frac{1}{q}}\left[\mu\left(\delta^{-j} B\right)\right]^{\frac{1}{q^{\prime}}} \\
& \lesssim \delta^{j \epsilon} \delta^{-(j-k) \omega}\left\|\mathbf{1}_{B}\right\|_{L^{\varphi}(\mathcal{X})}^{-1}=: \widetilde{C}_{3} \delta^{j \epsilon} \delta^{-(j-k) \omega}\left\|\mathbf{1}_{B}\right\|_{L^{\varphi}(\mathcal{X})}^{-1}
\end{aligned}
$$

where $\widetilde{C}_{3}$ is a fixed positive constant.

By (6.13), we obtain

$$
\begin{aligned}
\left\|\sum_{k=0}^{\infty} \sum_{j=k}^{\infty} b_{k, j}\right\|_{L^{\infty}(\mathcal{X})} & \leq \sum_{k=0}^{\infty} \sum_{j=k}^{\infty}\left\|b_{k, j}\right\|_{L^{\infty}(\mathcal{X})} \lesssim \sum_{k=0}^{\infty} \sum_{j=k}^{\infty} \delta^{j \epsilon} \delta^{-(j-k) \omega}\left\|\mathbf{1}_{B}\right\|_{L^{\varphi}(\mathcal{X})}^{-1} \\
& \lesssim\left\|\mathbf{1}_{B}\right\|_{L^{\varphi}(\mathcal{X})}^{-1} \sum_{k=0}^{\infty} \delta^{k \epsilon} \sum_{j=k}^{\infty} \delta^{(j-k)(\epsilon-\omega)} \lesssim\left\|\mathbf{1}_{B}\right\|_{L^{\varphi}(\mathcal{X})}^{-1}<\infty
\end{aligned}
$$

which further implies that $\sum_{k=0}^{\infty} \sum_{j=k}^{\infty} b_{k, j}$ converges in $L^{\infty}(\mathcal{X})$ and hence in $\left(\stackrel{\mathcal{G}}{0}_{0}^{\eta}(\varrho, \vartheta)\right)^{\prime}$.

From (6.13) again, it follows that $\frac{\delta^{-j \epsilon}}{\widetilde{C}_{3}} \delta^{(j-k) \omega} \frac{\left\|\mathbf{1}_{B}\right\|_{L^{\varphi}(\mathcal{X})}}{\left\|\mathbf{1}_{\delta^{-k_{B}}}\right\|_{L^{\varphi}(\mathcal{X})}}$ is a $(\varphi, \infty)$-atom. Meanwhile, by $\epsilon \in\left(\omega \frac{q(\varphi)}{i(\varphi)}, \infty\right)$, we know that there exist $q_{0} \in(q(\varphi), \infty)$ and $p_{0} \in(0, i(\varphi))$ such that $\epsilon \in\left(\omega \frac{q_{0}}{p_{0}}, \infty\right)$, which also implies that $\varphi \in \mathbf{A}_{q_{0}}(\mathcal{X})$ and $\varphi$ is of uniformly lower type $p_{0}$.

From the convergences of both $\sum_{k=0}^{\infty} M_{k}$ and $\sum_{k=0}^{\infty} \sum_{j=k}^{\infty} b_{k, j},(6.12)$ and (6.10), we deduce that

$$
m=\sum_{k=0}^{\infty} M_{k}+\sum_{k=0}^{\infty} \sum_{j=k}^{\infty} b_{j, k}
$$

converges in $\left(\mathcal{G}_{0}^{\eta}(\varrho, \vartheta)\right)^{\prime}$. By these, the above claim and Lemma 2.6(iv), we conclude that, for any $\lambda \in(0, \infty)$,

$$
\begin{aligned}
& \sum_{k=0}^{\infty} \varphi\left(\delta^{-k} B, \lambda\left\|M_{k}\right\|_{L_{\varphi}^{\widetilde{q}}\left(\delta^{-k} B\right)}\right)+\sum_{k=0}^{\infty} \sum_{j=k}^{\infty} \varphi\left(\delta^{-k} B, \lambda\left\|b_{k, j}\right\|_{L^{\infty}(\mathcal{X})}\right) \\
& \lesssim \sum_{k=0}^{\infty} \varphi\left(\delta^{-k} B, \lambda \delta^{k \epsilon}\left\|\mathbf{1}_{B}\right\|_{L^{\varphi}(\mathcal{X})}^{-1}\right)+\sum_{k=0}^{\infty} \sum_{j=k}^{\infty} \varphi\left(\delta^{-k} B, \lambda \delta^{-(j-k) \omega} \delta^{j \epsilon}\left\|\mathbf{1}_{B}\right\|_{L^{\varphi}(\mathcal{X})}^{-1}\right)
\end{aligned}
$$




$$
\begin{aligned}
& \lesssim \sum_{k=0}^{\infty} \delta^{-k \omega q_{0}} \delta^{k p_{0} \epsilon} \varphi\left(B, \lambda\left\|\mathbf{1}_{B}\right\|_{L^{\varphi}(\mathcal{X})}^{-1}\right)+\sum_{k=0}^{\infty} \sum_{j=k}^{\infty} \delta^{-k \omega q_{0}} \delta^{-(j-k) \omega p_{0}} \delta^{j p_{0} \epsilon} \varphi\left(B, \lambda\left\|\mathbf{1}_{B}\right\|_{L^{\varphi}(\mathcal{X})}^{-1}\right) \\
& \sim \sum_{k=0}^{\infty} \delta^{k p_{0}\left(\epsilon-\omega \frac{q_{0}}{p_{0}}\right)} \varphi\left(B, \lambda\left\|\mathbf{1}_{B}\right\|_{L^{\varphi}(\mathcal{X})}^{-1}\right) \\
&+\sum_{k=0}^{\infty} \delta^{k p_{0}\left(\epsilon-\omega \frac{q_{0}}{p_{0}}\right)} \sum_{j=k}^{\infty} \delta^{(j-k)(\epsilon-\omega) p_{0}} \varphi\left(B, \lambda\left\|\mathbf{1}_{B}\right\|_{L^{\varphi}(\mathcal{X})}^{-1}\right) \\
& \lesssim \varphi\left(B, \lambda\left\|\mathbf{1}_{B}\right\|_{L^{\varphi}(\mathcal{X})}^{-1}\right) .
\end{aligned}
$$

Let $f \in H_{\text {mol }}^{\varphi, q, \epsilon}(\mathcal{X})$. We know that there exist numbers $\left\{\lambda_{j}\right\}_{j=1}^{\infty} \subset \mathbf{C}$ and a sequence $\left\{m_{j}\right\}_{j=1}^{\infty}$ of $(\varphi, q, \epsilon)$-molecules such that

$$
f=\sum_{j=1}^{\infty} \lambda_{j} m_{j} \quad \text { in } \quad\left(\mathcal{G}_{0}^{\eta}(\varrho, \vartheta)\right)^{\prime}
$$

and

$$
\|f\|_{H_{\mathrm{mol}}^{\varphi, q, \epsilon}(\mathcal{X})} \sim \Lambda_{q}\left(\left\{\lambda_{j} m_{j}\right\}_{j=1}^{\infty}\right) .
$$

Then, by the above arguments, we find that, for any $j \in \mathbf{N}$, there exist a sequence $\left\{a_{j, k}\right\}_{k \in \mathbf{N}}$ of $(\varphi, q)$-atoms and numbers $\left\{\mu_{j, k}\right\}_{k \in \mathbf{N}} \subset \mathbf{C}$ such that $m_{j}=\sum_{k=0}^{\infty} \mu_{j, k} a_{j, k}$ in $\left(\mathcal{G}_{0}^{\eta}(\varrho, \vartheta)\right)^{\prime}$. Thus, we have

$$
f=\sum_{j=1}^{\infty} \sum_{k=0}^{\infty} \lambda_{j} \mu_{j, k} a_{j, k} \quad \text { in }\left(\stackrel{\circ}{\mathcal{G}}_{0}^{\eta}(\varrho, \vartheta)\right)^{\prime},
$$

which, together with Lemma 2.8(i), (6.14) and (6.15), further implies that

$$
\|f\|_{H_{\text {at }}^{\varphi}(\mathcal{X})} \lesssim \Lambda_{q}\left(\left\{\lambda_{j} \mu_{j, k} a_{j, k}\right\}_{j, k=1}^{\infty}\right) \lesssim \Lambda_{q}\left(\left\{\lambda_{j} m_{j}\right\}_{j=1}^{\infty}\right) \sim\|f\|_{H_{\text {mol }}^{\varphi, q, \epsilon}(\mathcal{X})} .
$$

This finishes the proof of $H_{\text {mol }}^{\varphi, q, \epsilon}(\mathcal{X}) \subset H_{\text {at }}^{\varphi}(\mathcal{X})$ and hence of Theorem 6.8.

6.3. Atomic characterizations of $H^{\varphi}(\mathcal{X})$. In this section, we aim to obtain the atomic characterizations of $H^{\varphi}(\mathcal{X})$.

The following notion of homogeneous atomic Musielak-Orlicz Hardy spaces is taken from [21].

Definition 6.9. (i) Let $(\varphi, q)$ be admissible as in Definition 5.2 and $\varrho, \vartheta \in$ $\left(\omega\left[\frac{q(\varphi)}{p}-1\right], \eta\right)$. The homogeneous atomic Musielak-Orlicz Hardy space $\stackrel{\circ}{H}_{\mathrm{at}}^{\varphi, q}(\mathcal{X})$ is defined in the same way as $H_{\mathrm{at}}^{\varphi, q}(\mathcal{X})$ in Definition 5.2 with $\left(\mathcal{G}_{0}^{\eta}(\varrho, \vartheta)\right)^{\prime}$ replaced by $\left(\stackrel{\mathcal{G}}{0}_{0}^{\eta}(\varrho, \vartheta)\right)^{\prime}$.

(ii) Let $q \in(1, \infty), s \in \mathbf{Z}_{+}$and $\epsilon \in(0, \infty)$. The homogeneous molecular MusielakOrlicz Hardy space $\stackrel{\circ}{H}_{\text {mol }}^{\varphi, q, \epsilon}(\mathcal{X})$ is defined in the same way as $H_{\text {mol }}^{\varphi, q, \epsilon}(\mathcal{X})$ in Definition 6.7 with $\left(\mathcal{G}_{0}^{\eta}(\varrho, \vartheta)\right)^{\prime}$ replaced by $\left(\stackrel{\mathcal{G}}{0}_{0}^{\eta}(\varrho, \vartheta)\right)^{\prime}$.

Then we introduce notions of adapted atomic Musielak-Orlicz Hardy spaces, which are generalized from the Euclidean case in [18] to any space of homogeneous type.

Definition 6.10. (I) Let $(\varphi, q)$ be admissible as in Definition 5.2 and $\varrho, \vartheta \in$ $\left(\omega\left[\frac{q(\varphi)}{p}-1\right], \eta\right)$. A function $a \in L^{q}(\mathcal{X})$ is called an adapted $(\varphi, q)_{A^{-}}$atom 
supported in a ball $B$ if Definition 5.2(iii) holds true and, instead of (i) and (ii) of Definition 5.2, $a$ satisfies

(I) 1 supp $a \subset B$;

$(\mathrm{I})_{2}\|a\|_{L^{q}(\mathcal{X})} \leq \frac{|B|^{1 / q}}{\left\|\mathbf{1}_{B}\right\|_{L^{\varphi}(\mathcal{X})}}$.

(II) The adapted homogeneous atomic Musielak-Orlicz Hardy space $\stackrel{\circ}{H}_{\mathrm{at}, \mathrm{A}}^{\varphi, q}(\mathcal{X})$ is defined to be the set of all $f \in\left(\stackrel{\mathcal{G}}{0}_{0}^{\eta}(\varrho, \vartheta)\right)^{\prime}$ satisfying that there exist a sequence $\left\{a_{j}\right\}_{j \in \mathbf{N}}$ of some $(\varphi, q)_{A}$-atoms supported, respectively, in balls $\left\{B_{j}\right\}_{j \in \mathbf{N}}$ and $\left\{\lambda_{j}\right\}_{j \in \mathbf{N}} \subset \mathbf{C}$ such that

$$
\sum_{j=1}^{\infty} \varphi\left(B_{j}, \frac{\left|\lambda_{j}\right|}{\left\|\mathbf{1}_{B_{j}}\right\|_{L^{\varphi}(\mathcal{X})}}\right)<\infty
$$

and $f=\sum_{j=1}^{\infty} \lambda_{j} a_{j}$ in $\left(\stackrel{\circ}{\mathcal{G}}_{0}^{\eta}(\varrho, \vartheta)\right)^{\prime}$. Moreover, let

$$
\widetilde{\Lambda}_{q}\left(\left\{\lambda_{j} a_{j}\right\}_{j=1}^{\infty}\right):=\inf \left\{\lambda \in(0, \infty): \sum_{j=1}^{\infty} \varphi\left(B_{j}, \frac{\left|\lambda_{j}\right|}{\lambda\left\|\mathbf{1}_{B_{j}}\right\|_{L^{\varphi}(\mathcal{X})}}\right) \leq 1\right\}
$$

and then define

$$
\|f\|_{H_{\mathrm{at}, \mathrm{A}}^{\varphi, q}(\mathcal{X})}:=\inf \left\{\widetilde{\Lambda}_{q}\left(\left\{\lambda_{j} a_{j}\right\}_{j=1}^{\infty}\right)\right\}
$$

where the last infimum is taken over all decompositions of $f$ as above.

(III) The adapted atomic Musielak-Orlicz Hardy space $H_{\mathrm{at}, \mathrm{A}}^{\varphi, q}(\mathcal{X})$ is defined similar to $\stackrel{\circ}{H}_{\mathrm{at}, \mathrm{A}}^{\varphi, q}(\mathcal{X})$ but with the distribution $\left(\stackrel{\mathcal{G}}{0}_{0}^{\eta}(\varrho, \vartheta)\right)^{\prime}$ replaced by $\left(\mathcal{G}_{0}^{\eta}(\varrho, \vartheta)\right)^{\prime}$.

Now, we show that $H_{\mathrm{at}, \mathrm{A}}^{\varphi, q}(\mathcal{X})$ and $\stackrel{\circ}{H}_{\mathrm{at}, \mathrm{A}}^{\varphi, q}(\mathcal{X})$ coincide, respectively, with $H_{\mathrm{at}}^{\varphi, q}(\mathcal{X})$ and $\stackrel{\circ}{H}_{\mathrm{at}}^{\varphi, q}(\mathcal{X})$, which are generalizations of the Euclidean case in [18, Theorem 2.12] to any space of homogeneous type.

Theorem 6.11. Suppose that $(\varphi, q)$ is admissible as in Definition 5.2, and $\varrho, \vartheta \in$ $\left(\omega\left[\frac{q(\varphi)}{p}-1\right], \eta\right)$ with $q(\varphi)$ as in (3.16). Then

(i) $H_{\mathrm{at}, \mathrm{A}}^{\varphi, q}(\mathcal{X})=H_{\mathrm{at}}^{\varphi, q}(\mathcal{X})$ with equivalent quasi-norms;

(ii) $\stackrel{\circ}{H}_{\mathrm{at}, \mathrm{A}}^{\varphi, q}(\mathcal{X})=\stackrel{\circ}{H}_{\mathrm{at}}^{\varphi, q}(\mathcal{X})$ with equivalent quasi-norms.

Proof. We only prove (i), because the proof of (ii) is similar. To show (i), we notice that any $(\varphi, \infty)_{A}$-atom in Definition 6.10 is the same as a $(\varphi, \infty)$-atom in Definition 5.2 and is also a $(\varphi, q)_{A}$-atom in Definition 6.10. Thus, by Remark 5.5, we have

$$
H_{\mathrm{at}}^{\varphi, q}(\mathcal{X})=H_{\mathrm{at}}^{\varphi, \infty}(\mathcal{X})=H_{\mathrm{at}, \mathrm{A}}^{\varphi, \infty}(\mathcal{X}) \subset H_{\mathrm{at}, \mathrm{A}}^{\varphi, q}(\mathcal{X}) .
$$

On another hand, observe that any $(\varphi, q)_{A}$-atom in Definition 6.10 is also a $(\varphi, q, \epsilon)$-molecule in Definition 6.7, which, combined with Theorem 6.8, further implies that $H_{\mathrm{at}, \mathrm{A}}^{\varphi, q}(\mathcal{X}) \subset H_{\text {mol }}^{\varphi, q, \epsilon}(\mathcal{X})=H_{\mathrm{at}}^{\varphi, q}(\mathcal{X})$. This finishes the proof of Theorem 6.11 .

The following relationship between $H_{\mathrm{at}}^{\varphi, q}(\mathcal{X})$ and $\stackrel{\circ}{H}_{\mathrm{at}}^{\varphi, q}(\mathcal{X})$ is a generalization of the corresponding results on Hardy spaces $H^{p}(\mathcal{X})$ in [21, Theorem 5.4] [in this case, $\varphi$ is the same as in (4.2)].

Proposition 6.12. Suppose that $(\varphi, q)$ is admissible as in Definition 5.2 and $\varrho, \vartheta \in\left(\omega\left[\frac{q(\varphi)}{p}-1\right], \eta\right)$. Then $\stackrel{\circ}{\mathrm{at}}_{\mathrm{\varphi}, q}(\mathcal{X})=H_{\mathrm{at}}^{\varphi, q}(\mathcal{X})$ with equivalent (quasi-)norms. More precisely, for any $f \in H_{\mathrm{at}}^{\varphi, q}(\mathcal{X})$, the restriction of $f$ on $\left(\dot{\mathcal{G}}_{0}^{\eta}(\varrho, \vartheta)\right)^{\prime}$ belongs to 
Real-variable characterizations of Musielak-Orlicz Hardy spaces on spaces of homogeneous type 381 $\stackrel{\circ}{H}_{\text {at }}^{\varphi, q}(\mathcal{X})$; Conversely, for any $f \in \stackrel{\circ}{H}_{\text {at }}^{\varphi, q}(\mathcal{X})$, there exists a unique $\tilde{f} \in H_{\text {at }}^{\varphi, q}(\mathcal{X})$ such that $\widetilde{f}=f$ in $\left(\dot{\mathcal{G}}_{0}^{\eta}(\varrho, \vartheta)\right)^{\prime}$. lemma.

Before proving Proposition 6.12, we need to establish the following auxiliary

Lemma 6.13. There exists a positive constant $C$ such that, for any sequence $\left\{\lambda_{j}\right\}_{j \in \mathbf{N}} \subset \mathbf{C}$ and for any sequence $\left\{a_{j}\right\}_{j \in \mathbf{N}}$ of $(\varphi, q)$-atoms supported, respectively, in balls $\left\{B_{j}\right\}_{j \in \mathbf{N}}$,

$$
\sum_{j=1}^{\infty}\left|\lambda_{j}\right| \leq C \widetilde{\Lambda}_{q}\left(\left\{\lambda_{j} a_{j}\right\}_{j \in \mathbf{N}}\right)
$$

where $\widetilde{\Lambda}_{q}$ is as in (6.16).

Proof. Let $\lambda:=\sum_{j=1}^{\infty}\left|\lambda_{j}\right|$. By the uniformly upper-type 1 property of $\varphi$ and Lemma 2.8(ii), we have

$$
\begin{aligned}
\sum_{j=1}^{\infty} \int_{B_{j}} \varphi\left(x, \frac{\left|\lambda_{j}\right|}{\lambda\left\|\mathbf{1}_{B_{j}}\right\|_{L^{\varphi}(\mathcal{X})}}\right) d \mu(x) & \gtrsim \sum_{j=1}^{\infty} \frac{\left|\lambda_{j}\right|}{\lambda} \int_{B_{j}} \varphi\left(x, \frac{1}{\left\|\mathbf{1}_{B_{j}}\right\|_{L^{\varphi}(\mathcal{X})}}\right) d \mu(x) \\
& \sim \sum_{j=1}^{\infty} \frac{\left|\lambda_{j}\right|}{\lambda} \sim 1,
\end{aligned}
$$

which implies that

$$
\sum_{j=1}^{\infty}\left|\lambda_{j}\right| \lesssim \widetilde{\Lambda}_{q}\left(\left\{\lambda_{j} a_{j}\right\}_{j \in \mathbf{N}}\right) .
$$

This finishes the proof of Lemma 6.13

We are now ready to prove Proposition 6.12.

Proof of Proposition 6.12. By $\left(\mathcal{G}_{0}^{\eta}(\varrho, \vartheta)\right)^{\prime} \subset\left(\dot{\mathcal{G}}_{0}^{\eta}(\varrho, \vartheta)\right)^{\prime}$ with $\varrho, \vartheta \in\left(\omega\left[\frac{q(\varphi)}{p}-1\right], \eta\right)$, we easily obtain $H_{\mathrm{at}}^{\varphi, q}(\mathcal{X}) \subset \stackrel{\circ}{H}_{\mathrm{at}}^{\varphi, q}(\mathcal{X})$. Thus, it suffices to show that $\stackrel{\circ}{H}_{\mathrm{at}}^{\varphi, q}(\mathcal{X}) \subset$ $H_{\mathrm{at}}^{\varphi, q}(\mathcal{X})$. Indeed, let $f \in \stackrel{\circ}{H}_{\mathrm{at}}^{\varphi, q}(\mathcal{X})$. Then, by Definition 6.9 , we know that $f \in$ $\left(\dot{\mathcal{G}}_{0}^{\eta}(\varrho, \vartheta)\right)^{\prime}$ and there exist a sequence $\left\{a_{j}\right\}_{j \in \mathbf{N}}$ of $(\varphi, q)$-atoms supported, respectively, in balls $\left\{B_{j}\right\}_{j \in \mathbf{N}}$ as in Definition 5.2, and numbers $\left\{\lambda_{j}\right\}_{j \in \mathbf{N}} \subset \mathbf{C}$ such that

$$
f=\sum_{j=1}^{\infty} \lambda_{j} a_{j} \quad \text { in }\left(\stackrel{\circ}{\mathcal{G}}_{0}^{\eta}(\varrho, \vartheta)\right)^{\prime}
$$

and

$$
\widetilde{\Lambda}_{q}\left(\left\{\lambda_{j} a_{j}\right\}_{j \in \mathbf{N}}\right) \lesssim\|f\|_{\stackrel{\circ}{\mathrm{at}, \mathrm{A}}_{\varphi, q}(\mathcal{X})},
$$

which, combined with Theorem 6.11(ii), implies that

$$
\widetilde{\Lambda}_{q}\left(\left\{\lambda_{j} a_{j}\right\}_{j \in \mathbf{N}}\right) \lesssim\|f\|_{\stackrel{\circ}{\mathrm{at}}^{\varphi, q}(\mathcal{X})} .
$$

Now, for any $h \in \mathcal{G}_{0}^{\eta}(\varrho, \vartheta)$, define

$$
\langle\tilde{f}, h\rangle:=\sum_{j=1}^{\infty} \lambda_{j}\left\langle a_{j}, h\right\rangle .
$$


From Lemmas 4.9 and 6.13 , Theorem 5.4 and the fact that $\left\|a_{j}\right\|_{H_{\mathrm{at}}^{\varphi, q}(\mathcal{X})} \lesssim 1$ and (6.17), it follows that

$$
\begin{aligned}
|\langle\widetilde{f}, h\rangle| & \leq \sum_{j=1}^{\infty}\left|\lambda_{j}\right|\left|\left\langle a_{j}, h\right\rangle\right| \lesssim \sum_{j=1}^{\infty}\left|\lambda_{j}\right|\|h\|_{\mathcal{G}_{0}^{\eta}(\varrho, \vartheta)}\left\|a_{j}\right\|_{H^{*, \varphi}(\mathcal{X})} \\
& \sim \sum_{j=1}^{\infty}\left|\lambda_{j}\right|\|h\|_{\mathcal{G}_{0}^{\eta}(\varrho, \vartheta)}\left\|a_{j}\right\|_{H_{\mathrm{at}}^{\varphi, q}(\mathcal{X})} \lesssim \sum_{j=1}^{\infty}\left|\lambda_{j}\right|\|h\|_{\mathcal{G}_{0}^{\eta}(\varrho, \vartheta)} \\
& \lesssim\|h\|_{\mathcal{G}_{0}^{\eta}(\varrho, \vartheta)} \widetilde{\Lambda}_{q}\left(\left\{\lambda_{j} a_{j}\right\}_{j \in \mathbf{N}}\right) \lesssim\|h\|_{\mathcal{G}_{0}^{\eta}(\varrho, \vartheta)}\|f\|_{\stackrel{H}{\mathrm{at}}^{\varphi, q}(\mathcal{X})}
\end{aligned}
$$

This shows that $\tilde{f} \in\left(\mathcal{G}_{0}^{\eta}(\varrho, \vartheta)\right)^{\prime}$ and $\tilde{f}=\sum_{j=1}^{\infty} \lambda_{j} a_{j}$ in $\left(\mathcal{G}_{0}^{\eta}(\varrho, \vartheta)\right)^{\prime}$. Moreover, $\tilde{f}=f$ in $\left(\stackrel{\mathcal{G}}{0}_{0}^{\eta}(\varrho, \vartheta)\right)^{\prime}, \tilde{f} \in H^{*, \varphi}(\mathcal{X})$ and

$$
\|\tilde{f}\|_{H_{\mathrm{at}}^{\varphi, q}(\mathcal{X})} \lesssim\|f\|_{H_{\mathrm{at}}^{\varphi, q}(\mathcal{X})} .
$$

Then we are left to show the uniqueness of the extension $\widetilde{f}$ of $f$. Indeed, suppose that there exists another extension of $f$, say, $\widetilde{g} \in H_{\mathrm{at}}^{\varphi, q}(\mathcal{X})$. Then $\widetilde{g}=\widetilde{f}$ on $\left(\dot{\mathcal{G}}_{0}^{\eta}(\varrho, \vartheta)\right)^{\prime}$, which, together with [21, Lemma 5.2], implies that $\widetilde{f}-\widetilde{g}$ is a constant, denoted by $\widetilde{C}$. If $\widetilde{C} \neq 0$, then it contradicts to the fact that no non-zero constant belongs to $H_{\theta}^{\varphi}(\mathcal{X})=H_{\text {at }}^{\varphi, q}(\mathcal{X})$. Therefore, $\widetilde{C}=0$, which further implies that $\widetilde{f} \in H_{\text {at }}^{\varphi, q}(\mathcal{X})$ is the unique extension of $f$. This finishes the proof of Proposition 6.12.

Remark 6.14. Let $\varphi, q$ and $\epsilon$ be as in Theorem 6.8. From the proof of Theorem 6.8, it follows that $\stackrel{\leftrightarrow}{H}_{\text {mol }}^{\varphi, q}(\mathcal{X})=\stackrel{\circ}{H}_{\text {at }}^{\varphi, q}(\mathcal{X})$ with equivalent quasi-norms.

Now, we state the main result of this subsection, which is a generalization of the corresponding results on Hardy spaces $H^{p}(\mathcal{X})$ in [27, Theorem 5.9] [in this case, $\varphi$ is the same as in (4.2)].

Theorem 6.15. Assume that $(\varphi, q)$ is admissible as in Definition 5.2 and $\varrho, \vartheta \in$ $\left(\omega\left[\frac{q(\varphi)}{p}-1\right], \eta\right)$ with $q(\varphi) \in\left[1, \frac{p(\eta+\omega)}{\omega}\right)$ as in (3.16). Then, as subspaces of $\left(\stackrel{\mathcal{G}}{0}_{0}^{\eta}(\varrho, \vartheta)\right)^{\prime}$, $H^{\varphi}(\mathcal{X})$ and $\stackrel{\circ}{H}_{\mathrm{at}}^{\varphi, q}(\mathcal{X})$ coincide with equivalent (quasi-)norms.

Proof. By Theorem 6.11(ii), we have $\stackrel{\circ}{H}_{\mathrm{at}}^{\varphi, q}(\mathcal{X})=\stackrel{\circ}{H_{\mathrm{at}, \mathrm{A}}^{\varphi, q}}(\mathcal{X})$ with equivalent

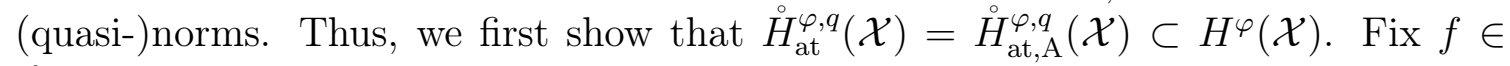
$\stackrel{\circ}{H}_{\text {at, }}^{\varphi, q}(\mathcal{X})$. Then, by Definition 6.10 , we know that there exist sequences $\left\{\lambda_{j}\right\}_{j \in \mathbf{N}} \subset \mathbf{C}$ and $\left\{a_{j}\right\}_{j \in \mathbf{N}}$ of adapted $(\varphi, q)_{A}$-atoms supported, respectively, in balls $\left\{B_{j}\right\}_{j \in \mathbf{N}}:=$ $\left\{B\left(x_{j}, r_{j}\right)\right\}_{j \in \mathbf{N}}$ with $x_{j} \in \mathcal{X}$ and $r_{j} \in(0, \infty)$ for any $j \in \mathbf{N}$ as in Definition 6.10, such that $f=\sum_{j=1}^{\infty} \lambda_{j} a_{j}$ in $\left(\dot{\mathcal{G}}_{0}^{\eta}(\varrho, \vartheta)\right)^{\prime}$ with $\varrho, \vartheta \in\left(\omega\left[\frac{q(\varphi)}{p}-1\right], \eta\right)$, and

$$
\widetilde{\Lambda}_{q}\left(\left\{\lambda_{j} a_{j}\right\}_{j \in \mathbf{N}}\right) \lesssim\|f\|_{H_{\mathrm{at}, \mathrm{A}}^{\varphi, q}(\mathcal{X})} .
$$

Let $\theta \in(0, \infty)$. We aim to show that

$$
\left\|S_{\theta}(f)\right\|_{L^{\varphi}(\mathcal{X})} \lesssim \max \left\{\theta^{-\frac{\omega}{2}}, \theta^{(1+q) \omega+\eta}\right\}\|f\|_{\stackrel{H}{\mathrm{at}, \mathrm{A}}_{\varphi}^{\varphi, q}(\mathcal{X})} .
$$

To this end, by the proof of [27, Proposition 5.6], it suffices to prove that, for any $\theta \in[1, \infty)$,

$$
\left\|S_{\theta}(f)\right\|_{L^{\varphi}(\mathcal{X})} \lesssim \theta^{(1+q) \omega+\eta}\|f\|_{\stackrel{\circ}{\mathrm{at}, A}^{\varphi, q}(\mathcal{X})}
$$


Real-variable characterizations of Musielak-Orlicz Hardy spaces on spaces of homogeneous type 383

Indeed, we observe that

$$
S_{\theta}(f) \leq \sum_{j=1}^{\infty} S_{\theta}\left(\lambda_{j} a_{j}\right) \mathbf{1}_{4 A_{0}^{2} \theta B_{j}}+\sum_{j=1}^{\infty} S_{\theta}\left(\lambda_{j} a_{j}\right) \mathbf{1}_{\left(4 A_{0}^{2} \theta B_{j}\right)^{\mathrm{C}}}=: \mathrm{I}+\mathrm{II} .
$$

To estimate I, by some standard arguments, we reduce the proof to showing that, for any $(\varphi, q)_{A}$-atom $a$ supported in a ball $B:=B\left(x_{B}, r_{B}\right)$ with $x_{B} \in \mathcal{X}$ and $r_{B} \in(0, \infty)$, and $\lambda \in \mathbf{C}$,

$$
\mathrm{A}:=\int_{4 A_{0}^{2} \theta B} \varphi\left(x, S_{\theta}(\lambda a)(x)\right) d \mu(x) \lesssim \theta^{(1+q) \omega+\eta} \varphi\left(B, \frac{|\lambda|}{\left\|\mathbf{1}_{B}\right\|_{L^{\varphi}(\mathcal{X})}}\right) .
$$

To this end, since $\varphi$ is of uniformly upper type 1 and of uniformly lower type $p$, it follows that

$$
\begin{aligned}
\mathrm{A} \lesssim & \left\|\mathbf{1}_{B}\right\|_{L^{\varphi}(\mathcal{X})} \int_{4 A_{0}^{2} \theta B} \varphi\left(x, \frac{\lambda}{\left\|\mathbf{1}_{B}\right\|_{L^{\varphi}(\mathcal{X})}}\right) S_{\theta}(a)(x) d \mu(x) \\
& +\left\|\mathbf{1}_{B}\right\|_{L^{\varphi}(\mathcal{X})}^{p} \int_{4 A_{0}^{2} \theta B} \varphi\left(x, \frac{\lambda}{\left\|\mathbf{1}_{B}\right\|_{L^{\varphi}(\mathcal{X})}}\right)\left[S_{\theta}(a)(x)\right]^{p} d \mu(x)=: \mathrm{A}_{1}+\mathrm{A}_{2} .
\end{aligned}
$$

Now, we start to estimate $\mathrm{A}_{1}$. Choose $q \in[2, \infty)$ such that $q^{\prime}<r(\varphi)$ with $r(\varphi)$ as in (6.9). Thus, $\varphi \in \mathbf{R H}_{q^{\prime}}(\mathcal{X})$. Moreover, from the boundedness of $S_{\theta}$ on $L^{q}(\mathcal{X})$ (see, for instance, the proof of [25, Proposition 2.17]), we deduce that

$$
\left\|S_{\theta}(a)\right\|_{L^{q}(\mathcal{X})} \lesssim \theta^{\omega}\|a\|_{L^{q}(\mathcal{X})} \lesssim \theta^{\omega} \frac{\left[\mu\left(B_{j}\right)\right]^{1 / q}}{\left\|\mathbf{1}_{B_{j}}\right\|_{L^{\varphi}(\mathcal{X})}}
$$

which, combined with the Hölder inequality, $\varphi \in \mathbf{R H}_{q^{\prime}}(\mathcal{X})$ and Lemma 2.6(iv), implies that

$$
\begin{aligned}
\mathrm{A}_{1} & \lesssim\left\|\mathbf{1}_{B}\right\|_{L^{\varphi}(\mathcal{X})}\left\|S_{\theta}(a)\right\|_{L^{q}(\mathcal{X})}\left\{\int_{4 A_{0}^{2} \theta B}\left[\varphi\left(x, \frac{|\lambda|}{\left\|\mathbf{1}_{B}\right\|_{L^{\varphi}(\mathcal{X})}}\right)\right]^{q^{\prime}} d \mu(x)\right\}^{1 / q^{\prime}} \\
& \lesssim \theta^{\omega}\left\|\mathbf{1}_{B}\right\|_{L^{\varphi}(\mathcal{X})} \frac{[\mu(B)]^{1 / q}}{\left\|\mathbf{1}_{B_{j}}\right\|_{L^{\varphi}(\mathcal{X})}}[\mu(B)]^{-1 / q} \varphi\left(4 A_{0}^{2} \theta B, \frac{|\lambda|}{\left\|\mathbf{1}_{B}\right\|_{L^{\varphi}(\mathcal{X})}}\right) \\
& \lesssim \theta^{(1+q) \omega} \varphi\left(B, \frac{|\lambda|}{\left\|\mathbf{1}_{B}\right\|_{L^{\varphi}(\mathcal{X})}}\right) .
\end{aligned}
$$

To deal with $\mathrm{A}_{2}$, we first observe that $q / p \geq q$ and hence $q^{\prime} \geq(q / p)^{\prime}$, which, together with Lemma 2.6(ii), implies that $\mathbf{R H}_{q^{\prime}}(\mathcal{X}) \subset \mathbf{R H}_{(q / p)^{\prime}}(\mathcal{X})$. By this, the Hölder inequality and Lemma 2.6(iv), we conclude that

$$
\begin{aligned}
\mathrm{A}_{2} & \lesssim\left\|\mathbf{1}_{B}\right\|_{L^{\varphi}(\mathcal{X})}^{p}\left\|S_{\theta}(a)\right\|_{L^{q}(\mathcal{X})}^{p}\left\{\int_{4 A_{0}^{2} \theta B}\left[\varphi\left(x, \frac{|\lambda|}{\left\|\mathbf{1}_{B}\right\|_{L^{\varphi}(\mathcal{X})}}\right)\right]^{(q / p)^{\prime}} d \mu(x)\right\}^{1 /(q / p)^{\prime}} \\
& \lesssim \theta^{\omega p}\left\|\mathbf{1}_{B}\right\|_{L^{\varphi}(\mathcal{X})}^{p} \frac{[\mu(B)]^{p / q}}{\left\|\mathbf{1}_{B_{j}}\right\|_{L^{\varphi}(\mathcal{X})}^{p}}[\mu(B)]^{-p / q} \varphi\left(4 A_{0}^{2} \theta B, \frac{|\lambda|}{\left\|\mathbf{1}_{B}\right\|_{L^{\varphi}(\mathcal{X})}}\right) \\
& \lesssim \theta^{(1+q) \omega} \varphi\left(B, \frac{|\lambda|}{\left\|\mathbf{1}_{B}\right\|_{L^{\varphi}(\mathcal{X})}}\right)
\end{aligned}
$$


which, combined with the estimate of $\mathrm{A}_{1}$, further implies that

$$
\mathrm{A} \lesssim \mathrm{A}_{1}+\mathrm{A}_{2} \lesssim \theta^{(1+q) \omega} \varphi\left(B, \frac{|\lambda|}{\left\|\mathbf{1}_{B}\right\|_{L^{\varphi}(\mathcal{X})}}\right)
$$

Now, we turn to estimate II. From an argument similar to that used in the proof of [27, Proposition 5.6] and (1.4), we deduce that, for any $x \in\left(4 A_{0}^{2} \theta B\right)^{\complement}$,

$$
\begin{aligned}
S_{\theta}(a)(x) & \lesssim \theta^{\omega+\eta} \frac{\mu(B)}{\left\|\mathbf{1}_{B}\right\|_{L^{\varphi}(\mathcal{X})}}\left[\frac{r}{d\left(x, x_{B}\right)}\right]^{\eta} \frac{1}{V\left(x, x_{B}\right)} \lesssim \theta^{\omega+\eta} \frac{1}{\left\|\mathbf{1}_{B}\right\|_{L^{\varphi}(\mathcal{X})}}\left[\frac{\mu(B)}{V\left(x, x_{B}\right)}\right]^{\frac{\eta}{\omega}+1} \\
& \lesssim \theta^{\omega+\eta} \frac{\left[M\left(\mathbf{1}_{B}\right)(x)\right]^{\frac{\eta}{\omega}+1}}{\left\|\mathbf{1}_{B}\right\|_{L^{\varphi}(\mathcal{X})}} .
\end{aligned}
$$

This, together with Theorem 6.6, further implies that

$$
\begin{aligned}
& \|\mathrm{II}\|_{L^{\varphi}(\mathcal{X})} \lesssim \theta^{\omega+\eta}\left\|\sum_{j=1}^{\infty}\left|\lambda_{j}\right| \frac{\left[M\left(\mathbf{1}_{B_{j}}\right)\right]^{\frac{\eta}{\omega}+1}}{\left\|\mathbf{1}_{B_{j}}\right\|_{L^{\varphi}(\mathcal{X})}}\right\|_{L^{\varphi}(\mathcal{X})} \\
& \sim \theta^{\omega+\eta}\left\|\sum_{j=1}^{\infty}\left[M\left(\left|\lambda_{j}\right|^{\frac{\omega}{\omega+\eta}} \frac{\mathbf{1}_{B_{j}}}{\left\|\mathbf{1}_{B_{j}}\right\|_{L^{\varphi}(\mathcal{X})}^{\frac{\omega}{\omega+\eta}}}\right)\right]^{\frac{\eta}{\omega}+1}\right\|_{L^{\varphi}(\mathcal{X})} \\
& \sim \theta^{\omega+\eta}\left\|\left\{\sum_{j=1}^{\infty}\left[M\left(\left|\lambda_{j}\right|^{\frac{\omega}{\omega+\eta}} \frac{\mathbf{1}_{B_{j}}}{\left\|\mathbf{1}_{B_{j}}\right\|_{L^{\varphi}(\mathcal{X})}^{\omega+\eta}}\right)\right]^{\frac{\eta}{\omega}+1}\right\}^{\frac{\omega}{\omega+\eta}}\right\|_{L^{\tilde{\varphi}}(\mathcal{X})}^{\frac{\omega+\eta}{\omega}} \\
& \lesssim \theta^{\omega+\eta}\left\|\left\{\sum_{j=1}^{\infty}\left|\lambda_{j}\right| \frac{\mathbf{1}_{B_{j}}}{\left\|\mathbf{1}_{B_{j}}\right\|_{L^{\varphi}(\mathcal{X})}}\right\}^{\frac{\omega}{\omega+\eta}}\right\|_{L^{\tilde{\varphi}}(\mathcal{X})}^{\frac{\omega+\eta}{\omega}} \sim \theta^{\omega+\eta}\left\|\sum_{j=1}^{\infty}\left|\lambda_{j}\right| \frac{\mathbf{1}_{B_{j}}}{\left\|\mathbf{1}_{B_{j}}\right\|_{L^{\varphi}(\mathcal{X})}}\right\|_{L^{\varphi}(\mathcal{X})} \\
& \lesssim \theta^{\omega+\eta} \widetilde{\Lambda}_{q}\left(\left\{\lambda_{j} a_{j}\right\}_{j \in \mathbf{N}}\right) \lesssim \theta^{\omega+\eta}\|f\|_{H_{\mathrm{at}, \mathrm{A}}^{\varphi, q}(\mathcal{X})} .
\end{aligned}
$$

From the estimates of I and II, we deduce that

$$
\left\|S_{\theta}(f)\right\|_{L^{\varphi}(\mathcal{X})} \lesssim\|\mathrm{I}\|_{L^{\varphi}(\mathcal{X})}+\|\mathrm{II}\|_{L^{\varphi}(\mathcal{X})} \lesssim \theta^{\omega+\eta}\|f\|_{\hat{H}_{\mathrm{at}, \mathrm{A}}^{\varphi, q}(\mathcal{X})},
$$

which implies (6.18) and hence shows $\stackrel{\circ}{H}_{\mathrm{at}}^{\varphi, q}(\mathcal{X}) \hookrightarrow H^{\varphi}(\mathcal{X})$. This is the desired conclusion.

Let $q$ and $\epsilon$ be as in Theorem 6.8. Then we show that $H^{\varphi}(\mathcal{X}) \subset \stackrel{\circ}{H}_{\text {mol }}^{\varphi, q}(\mathcal{X})=$ $\stackrel{\circ}{H}_{\mathrm{at}}^{\varphi, q}(\mathcal{X})$. Suppose that $f \in\left(\dot{\mathcal{G}}_{0}^{\eta}(\varrho, \vartheta)\right)^{\prime}$ belongs to $H^{\varphi}(\mathcal{X})$. By Theorem 6.3 , without loss of generality, we may choose the exp-ATI $\left\{D_{k}\right\}_{k \in \mathbf{Z}}$ as in Remark 4.4(i). Denote by $\mathcal{D}$ the set of all dyadic cubes. For any $k \in \mathbf{Z}$, define $\Omega_{k}:=\left\{x \in \mathcal{X}: S(f)(x)>2^{k}\right\}$ and

$$
\mathcal{D}_{k}:=\left\{Q \in \mathcal{D}: \mu\left(Q \cap \Omega_{k}\right)>\frac{1}{2} \mu(Q) \text { and } \mu\left(Q \cap \Omega_{k+1}\right) \leq \frac{1}{2} \mu(Q)\right\} .
$$

Obviously, for any $Q \in \mathcal{D}$, there exists a unique $k \in \mathbf{Z}$ such that $Q \in \mathcal{D}_{k}$. Denote by $\left\{Q_{\tau, k}^{j}\right\}_{\tau \in I_{j, k}}$ the set of all maximal cubes in $\mathcal{D}_{k}$ at level $j \in \mathbf{Z}$, where $I_{j, k} \subset \mathscr{A}_{j}$. The center of $Q_{\tau, k}^{j}$ is denoted by $z_{\tau, k}^{j}$. Then

$$
\mathcal{D}=\bigcup_{j, k \in \mathbf{Z}} \bigcup_{\tau \in I_{j, k}}\left\{Q \in \mathcal{D}_{k}: Q \subset Q_{\tau, k}^{j}\right\}
$$


Hereafter, we let $D_{Q}:=D_{\ell}$ when $Q:=Q_{\beta}^{\ell+1}$ for some $\ell \in \mathbf{Z}$ and $\beta \in \mathscr{A}_{\ell+1}$. We now show that

$$
f=\sum_{\ell \in \mathbf{Z}} D_{\ell} D_{\ell} f=\sum_{k \in \mathbf{Z}} \sum_{j \in \mathbf{Z}} \sum_{\tau \in I_{j, k}} \lambda_{\tau, k}^{j} b_{\tau, k}^{j},
$$

where all the equalities hold true in $\left(\stackrel{\mathcal{G}}{0}_{0}^{\eta}(\varrho, \vartheta)\right)^{\prime}$,

$$
\lambda_{\tau, k}^{j}:=2^{k}\left\|\mathbf{1}_{Q_{\tau, k}^{j}}\right\|_{L^{\varphi}(\mathcal{X})}
$$

and

$$
b_{\tau, k}^{j}(\cdot):=\frac{1}{\lambda_{\tau, k}^{j}} \sum_{\left\{Q \in \mathcal{D}_{k}, Q \subset Q_{\tau, k}^{j}\right\}} \int_{Q} D_{Q}(\cdot, y) D_{Q} f(y) d \mu(y) .
$$

Before proving (6.20), we first claim that $b_{\tau, k}^{j}$ is a positive harmless constant multiple of a $\left(\varphi, q, \epsilon_{0}\right)$-molecule, where $\epsilon_{0}$ is determined later, related to the ball $B_{\tau, k}^{j}:=B\left(z_{\tau, k}^{j}, 4 A_{0}^{2} \delta^{j-1}\right)$ as in Definition 6.7. Indeed, for any $Q \in \mathcal{D}_{k}$ and $Q \in$ $Q_{\tau, k}^{j}$, assume that $Q:=Q_{\alpha}^{\ell+1}$ for some $\ell \in \mathbf{Z}$ and $\alpha \in \mathscr{A}_{\ell+1}$. By the proof of $[27$, Proposition 5.7], we know that, for any $y \in Q$,

$$
\mu\left(B\left(y, \delta^{\ell}\right) \cap\left[Q_{\tau, k}^{j} \backslash \Omega_{k+1}\right]\right) \gtrsim V_{\delta^{\ell}}(y) .
$$

From this, the Fubini-Tonelli theorem, $V_{\delta^{\ell}}(x) \sim V_{\delta^{\ell}}(y)$ for any $x, y \in \mathcal{X}$ with $d(x, y)<\delta^{\ell}$, and the Hölder inequality, we deduce that, for any $h \in L^{q^{\prime}}(\mathcal{X})$ with $\|h\|_{L^{q^{\prime}}(\mathcal{X})} \leq 1$

$$
\begin{aligned}
& \left|\int_{\mathcal{X}} b_{\tau, k}^{j}(x) h(x) d \mu(x)\right| \\
\leq & \frac{1}{\lambda_{\tau, k}^{j}} \sum_{\left\{Q \in \mathcal{D}_{k}, Q \subset Q_{\tau, k}^{j}\right\}} \int_{Q}\left|D_{Q} f(y)\right|\left|D_{Q}^{*} h(y)\right| d \mu(y) \\
\lesssim & \frac{1}{\lambda_{\tau, k}^{j}} \sum_{\left\{Q \in \mathcal{D}_{k}, Q \subset Q_{\tau, k}^{j}\right\}} \int_{Q} \frac{\mu\left(B\left(y, \delta^{\ell}\right) \cap\left[Q_{\tau, k}^{j} \backslash \Omega_{k+1}\right]\right)}{V_{\delta^{\ell}}(y)}\left|D_{Q} f(y)\right|\left|D_{Q}^{*} h(y)\right| d \mu(y) \\
\sim & \frac{1}{\lambda_{\tau, k}^{j}} \sum_{\ell=j-1}^{\infty} \sum_{\alpha \in \mathscr{A}_{\ell+1}} \int_{\left\{Q_{\alpha}^{\ell+1} \in \mathcal{D}_{k}, Q_{\alpha}^{\ell+1} \subset Q_{\tau, k}^{j}\right\}} \frac{\mu\left(B\left(y, \delta^{\ell}\right) \cap\left[Q_{\tau, k}^{j} \backslash \Omega_{k+1}\right]\right)}{V_{\delta^{\ell}}(y)} \\
& \cdot\left|D_{\ell} f(y)\right|\left|D_{\ell}^{*} h(y)\right| d \mu(y) \\
\lesssim & \frac{1}{\lambda_{\tau, k}^{j}} \sum_{\ell=j-1}^{\infty} \int_{Q_{\tau, k}^{j}} \frac{\mu\left(B\left(y, \delta^{\ell}\right) \cap\left[Q_{\tau, k}^{j} \backslash \Omega_{k+1}\right]\right)}{V_{\delta^{\ell}}(y)}\left|D_{\ell} f(y)\right|\left|D_{\ell}^{*} h(y)\right| d \mu(y) \\
\sim & \frac{1}{\lambda_{\tau, k}^{j}} \sum_{\ell=j-1}^{\infty} \int_{Q_{\tau, k}^{j}} \int_{\mathcal{X}}^{\infty} \mathbf{1}_{B\left(y, \delta^{\ell}\right)}(x) \mathbf{1}_{Q_{\tau, k}^{j} \backslash \Omega_{k+1}}(x) \frac{d \mu(x)}{V_{\delta^{\ell}}(x)}\left|D_{\ell} f(y)\right|\left|D_{\ell}^{*} h(y)\right| d \mu(y) \\
\sim & \frac{1}{\lambda_{\tau, k}^{j}} \int_{Q_{\tau, k}^{j} \backslash \Omega_{k+1}} \sum_{\ell=j-1}^{\infty} \int_{Q_{\tau, k}^{j} \cap B\left(x, \delta^{\ell}\right)}\left|D_{\ell} f(y)\right|\left|D_{\ell}^{*} h(y)\right| \frac{d \mu(y)}{V_{\delta^{\ell}}(x)} d \mu(x)
\end{aligned}
$$




$$
\begin{aligned}
\lesssim & \frac{1}{\lambda_{\tau, k}^{j}} \int_{Q_{\tau, k}^{j} \backslash \Omega_{k+1}} \sum_{\ell=j-1}^{\infty}\left[\int_{B\left(x, \delta^{\ell}\right)}\left|D_{\ell} f(y)\right|^{2} \frac{d \mu(y)}{V_{\delta^{\ell}}(x)}\right]^{1 / 2}\left[\int_{B\left(x, \delta^{\ell}\right)}\left|D_{\ell}^{*} h(y)\right|^{2} \frac{d \mu(y)}{V_{\delta^{\ell}}(x)}\right]^{1 / 2} d \mu(x) \\
\lesssim & \frac{1}{\lambda_{\tau, k}^{j}} \int_{Q_{\tau, k}^{j} \backslash \Omega_{k+1}}\left[\sum_{\ell=j-1}^{\infty} \int_{B\left(x, \delta^{\ell}\right)}\left|D_{\ell} f(y)\right|^{2} \frac{d \mu(y)}{V_{\delta^{\ell}}(x)}\right]^{1 / 2} \\
& \cdot\left[\sum_{\ell=j-1}^{\infty} \int_{B\left(x, \delta^{\ell}\right)}\left|D_{\ell}^{*} h(y)\right|^{2} \frac{d \mu(y)}{V_{\delta^{\ell}}(x)}\right]^{1 / 2} d \mu(x) \\
\lesssim & \frac{1}{\lambda_{\tau, k}^{j}}\left\{\int_{Q_{\tau, k}^{j} \backslash \Omega_{k+1}}[S(f)(x)]^{q} d \mu(x)\right\}^{1 / q}\left\{\int_{Q_{\tau, k}^{j} \backslash \Omega_{k+1}}[\widetilde{S}(h)(x)]^{q^{\prime}} d \mu(x)\right\}^{1 / q^{\prime}} \\
\lesssim & \frac{2^{k}}{\lambda_{\tau, k}^{j}}\left[\mu\left(Q_{\tau, k}^{j}\right)\right]^{1 / q}\|\widetilde{S}(h)\|_{L^{q^{\prime}}(\mathcal{X})},
\end{aligned}
$$

where, for any $x \in \mathcal{X}$,

$$
\widetilde{S}(h)(x):=\left[\sum_{\ell=-\infty}^{\infty} \int_{B\left(x, \delta^{\ell}\right)}\left|D_{\ell}^{*} h(y)\right|^{2} \frac{d \mu(y)}{V_{\delta^{\ell}}(x)}\right]^{1 / 2}
$$

and $D_{\ell}^{*}$ is the adjoint operator on $L^{2}(\mathcal{X})$ of $D_{\ell}$ for any $\ell \in \mathbf{Z}$. By the proof of $[27$, Theorem 5.10], we conclude that

$$
\|\widetilde{S}(h)\|_{L^{q^{\prime}}(\mathcal{X})} \lesssim\|\widetilde{g}(h)\|_{L^{q^{\prime}}(\mathcal{X})}
$$

where $\widetilde{g}(h):=\left[\sum_{\ell \in \mathbf{Z}}\left|D_{\ell}^{*} h\right|^{2}\right]^{1 / 2}$. Following the arguments used in the proof of $[27$, Lemma 5.8], we know that $\widetilde{g}$ is bounded on $L^{2}(\mathcal{X})$, which, combined with the theory of vector-valued Calderón-Zygmund operators (see, for instance, [22]), implies that $\widetilde{g}$ is bounded on $L^{q^{\prime}}(\mathcal{X})$. Therefore, we have

$$
\left|\int_{\mathcal{X}} b_{\tau, k}^{j}(x) h(x) d \mu(x)\right| \lesssim \frac{\left[\mu\left(Q_{\tau, k}^{j}\right)\right]^{1 / q}}{\left\|\mathbf{1}_{Q_{\tau, k}^{j}}\right\|_{L^{\varphi}(\mathcal{X})}}\|h\|_{L^{q^{\prime}}(\mathcal{X})} \lesssim \frac{\left[\mu\left(Q_{\tau, k}^{j}\right)\right]^{1 / q}}{\left\|\mathbf{1}_{Q_{\tau, k}^{j}}\right\|_{L^{\varphi}(\mathcal{X})}} .
$$

Taking the supremum over all $h \in L^{q^{\prime}}(\mathcal{X})$ with $\|h\|_{L^{q^{\prime}}(\mathcal{X})} \leq 1$, we obtain

$$
\left\|b_{\tau, k}^{j}\right\|_{L^{q}(\mathcal{X})} \lesssim \frac{\left[\mu\left(Q_{\tau, k}^{j}\right)\right]^{1 / q}}{\left\|\mathbf{1}_{Q_{\tau, k}^{j}}\right\|_{L^{\varphi}(\mathcal{X})}}
$$

which, together with some arguments similar to those used in the proof of [27, Proposition 5.7], further implies that there exists $\epsilon_{0} \in\left(\omega\left[\frac{q(\varphi)}{p}-1\right], \varrho\right)$ such that

$$
\begin{aligned}
\left\|b_{\tau, k}^{j} \mathbf{1}_{U_{m}\left(B_{\tau, k}^{j}\right)}\right\|_{L^{q^{\prime}(\mathcal{X})}} & \lesssim \delta^{m \epsilon_{0}}\left[\frac{\mu\left(B_{\tau, k}^{j}\right)}{\mu\left(\delta^{-m} B_{\tau, k}^{j}\right)}\right]^{1 / 2} \frac{\left[\mu\left(B_{\tau, k}^{j}\right)\right]^{1 / q}}{\left\|\mathbf{1}_{B_{\tau, k}^{j}}\right\|_{L^{\varphi}(\mathcal{X})}} \\
& \lesssim \delta^{m \epsilon_{0}} \frac{\left[\mu\left(\delta^{-m} B_{\tau, k}^{j}\right)\right]^{1 / q}}{\left\|\mathbf{1}_{B_{\tau, k}^{j}}\right\|_{L^{\varphi}(\mathcal{X})}} .
\end{aligned}
$$

Thus, $b_{\tau, k}^{j}$ is a positive harmless constant multiple of a $\left(\varphi, q, \epsilon_{0}\right)$-molecule related to the ball $B_{\tau, k}^{j}:=B\left(z_{\tau, k}^{j}, 4 A_{0}^{2} \delta^{j-1}\right)$ as in Definition 6.7. This finishes the proof of the above claim. 
Moreover, by (6.23), (6.21), Lemma 2.6(iv) and some arguments similar to those used in the proof of [35, Lemma 5.4], we have

$$
\begin{aligned}
& \sum_{k \in \mathbf{Z}} \sum_{j \in \mathbf{Z}} \sum_{\tau \in I_{j, k}} \varphi\left(B_{\tau, k}^{j}, \frac{\left|\lambda_{\tau, k}^{j}\right|}{\left\|\mathbf{1}_{B_{\tau, k}^{j}}\right\|_{L^{\varphi}(\mathcal{X})}\|S(f)\|_{L^{\varphi}(\mathcal{X})}}\right) \\
& \lesssim \sum_{k \in \mathbf{Z}} \sum_{j \in \mathbf{Z}} \sum_{\tau \in I_{j, k}} \varphi\left(Q_{\tau, k}^{j}, \frac{\left|\lambda_{\tau, k}^{j}\right|}{\left\|\mathbf{1}_{Q_{\tau, k}^{j}}\right\|_{L^{\varphi}(\mathcal{X})}\|S(f)\|_{L^{\varphi}(\mathcal{X})}}\right) \\
& \lesssim \sum_{k \in \mathbf{Z}} \sum_{j \in \mathbf{Z}} \sum_{\tau \in I_{j, k}} \varphi\left(Q_{\tau, k}^{j}, \frac{2^{k}}{\|S(f)\|_{L^{\varphi}(\mathcal{X})}}\right) \\
& \lesssim \sum_{k \in \mathbf{Z}} \sum_{j \in \mathbf{Z}} \sum_{\tau \in I_{j, k}} \varphi\left(Q_{\tau, k}^{j} \cap \Omega_{k}, \frac{2^{k}}{\|S(f)\|_{L^{\varphi}(\mathcal{X})}}\right)\left[\frac{\mu\left(Q_{\tau, k}^{j}\right)}{\mu\left(Q_{\tau, k}^{j} \cap \Omega_{k}\right)}\right]^{p_{0}} \\
& \lesssim \sum_{k \in \mathbf{Z}} \varphi\left(\left[\bigcup_{j \in \mathbf{Z}} \bigcup_{\tau \in I_{j, k}} Q_{\tau, k}^{j}\right] \cap \Omega_{k}, \frac{2^{k}}{\|S(f)\|_{L^{\varphi}(\mathcal{X})}}\right) \\
& \lesssim \sum_{k \in \mathbf{Z}} \varphi\left(\Omega_{k}, \frac{2^{k}}{\|S(f)\|_{L^{\varphi}(\mathcal{X})}}\right) \lesssim \int_{\mathcal{X}} \varphi\left(x, \frac{S(f)}{\|S(f)\|_{L^{\varphi}(\mathcal{X})}}\right) d \mu(x) \sim 1,
\end{aligned}
$$

which implies that

$$
\Lambda_{q}\left(\left\{\lambda_{\tau, k}^{j} b_{\tau, k}^{j}\right\}_{j, \tau, k}\right) \lesssim\|S(f)\|_{L^{\varphi}(\mathcal{X})}
$$

where $\Lambda_{q}$ is as in (6.8).

Then we begin to prove (6.20). To this end, we need to show that

$$
\sum_{k \in \mathbf{Z}} \sum_{j \in \mathbf{Z}} \sum_{\tau \in I_{j, k}} \lambda_{\tau, k}^{j} b_{\tau, k}^{j}
$$

converges in $\left(\mathcal{G}_{0}^{\eta}(\varrho, \vartheta)\right)^{\prime}$ and hence in $\left(\stackrel{\mathcal{G}}{0}_{0}^{\eta}(\varrho, \vartheta)\right)^{\prime}$, because $\stackrel{\mathcal{G}}{0}_{0}^{\eta}(\varrho, \vartheta) \subset \mathcal{G}_{0}^{\eta}(\varrho, \vartheta)$. Indeed, let $\left\{\mathscr{E}_{N}\right\}_{N \in \mathbf{N}}$ be an increasing sequence of finite subset of $\mathscr{E}:=\{(k, j, \tau): k, j \in$ $\left.\mathbf{Z}, \tau \in I_{j, k}\right\}$ with $\mathscr{E}=\bigcup_{N \in \mathbf{N}} \mathscr{E}_{N}$. For any $N, M \in \mathbf{N}$ with $N>M$, by the above claim, Lemma 4.9, Theorem 6.8 and (6.24), we conclude that, for any $h \in \mathcal{G}_{0}^{\eta}(\varrho, \vartheta)$,

$$
\begin{aligned}
\sum_{(k, j, \tau) \in \mathscr{E}}\left|\lambda_{\tau, k}^{j}\right|\left|\left\langle b_{\tau, k}^{j}, h\right\rangle\right| & \lesssim \sum_{(k, j, \tau) \in \mathscr{E}}\left|\lambda_{\tau, k}^{j}\right|\left\|b_{\tau, k}^{j}\right\|_{H^{*, \varphi}(\mathcal{X})}\|h\|_{\mathcal{G}_{0}^{\eta}(\varrho, \vartheta)} \\
& \lesssim \sum_{(k, j, \tau) \in \mathscr{E}}\left|\lambda_{\tau, k}^{j}\right|\|h\|_{\mathcal{G}_{0}^{\eta}(\varrho, \vartheta)} \lesssim \Lambda_{q}\left(\left\{\lambda_{\tau, k}^{j} b_{\tau, k}^{j}\right\}_{j, \tau, k}\right)\|h\|_{\mathcal{G}_{0}^{\eta}(\varrho, \vartheta)} \\
& \lesssim\|S(f)\|_{L^{\varphi}(\mathcal{X})}\|h\|_{\mathcal{G}_{0}^{\eta}(\varrho, \vartheta)}<\infty
\end{aligned}
$$

which further implies that

$$
\left|\left\langle\sum_{\left\{(k, j, \tau) \in \mathscr{E}_{N} \backslash \mathscr{E}_{M}\right\}} \lambda_{\tau, k}^{j} b_{\tau, k}^{j}, h\right\rangle\right| \leq \sum_{\left\{(k, j, \tau) \in \mathscr{E}_{N} \backslash \mathscr{E}_{M}\right\}}\left|\lambda_{\tau, k}^{j}\right|\left|\left\langle b_{\tau, k}^{j}, h\right\rangle\right| \rightarrow 0 \quad \text { as } \quad M \rightarrow \infty .
$$

Thus, $\left\{\sum_{(k, j, \tau) \in \mathscr{E}_{N}} \lambda_{\tau, k}^{j} b_{\tau, k}^{j}\right\}_{N \in \mathbf{N}}$ is a Cauchy sequence in $\left(\mathcal{G}_{0}^{\eta}(\varrho, \vartheta)\right)^{\prime}$, which, combined with the completion of $\left(\mathcal{G}_{0}^{\eta}(\varrho, \vartheta)\right)^{\prime}$, implies that $\sum_{(k, j, \tau) \in \mathscr{E}_{N}} \lambda_{\tau, k}^{j} b_{\tau, k}^{j}$ converges to some $\widetilde{f} \in\left(\mathcal{G}_{0}^{\eta}(\varrho, \vartheta)\right)^{\prime}$. That is, $\sum_{k \in \mathbf{Z}} \sum_{j \in \mathbf{Z}} \sum_{\tau \in I_{j, k}} \lambda_{\tau, k}^{j} b_{\tau, k}^{j}$ converges in $\left(\mathcal{G}_{0}^{\eta}(\varrho, \vartheta)\right)^{\prime}$. 
By [24, Corollary 3.5], to prove (6.20), it suffices to show that, for any $\widetilde{k} \in \mathbf{Z}$ and $\widetilde{\beta} \in \mathscr{G}_{\widetilde{k}}$ with $\mathscr{G}_{\widetilde{k}}$ as in $(4.1)$ with $k$ replaced by $\widetilde{k}$,

$$
\left\langle f, \psi_{\widetilde{\beta}}^{\widetilde{k}}\right\rangle=\left\langle\sum_{\ell \in \mathbf{Z}} D_{\ell} D_{\ell} f, \psi_{\widetilde{\beta}}^{\widetilde{k}}\right\rangle=\left\langle\sum_{k \in \mathbf{Z}} \sum_{j \in \mathbf{Z}} \sum_{\tau \in I_{j, k}} \lambda_{\tau, k}^{j} b_{\tau, k}^{j}, \psi_{\widetilde{\beta}}^{\widetilde{k}}\right\rangle=\left\langle\widetilde{f}, \psi_{\widetilde{\beta}}^{\widetilde{k}}\right\rangle,
$$

where $\left\{\psi_{\widetilde{\beta}}^{\widetilde{k}}\right\}_{\widetilde{k} \in \mathbf{Z}, \widetilde{\beta} \in \mathscr{G}_{\widetilde{k}}}$ is as in Theorem 4.3 .

Let $\left\{\mathscr{I}_{N}\right\}_{N \in \mathbf{N}}$ be an increasing sequence of finite subset of $\mathscr{I}:=\left\{Q \in \mathcal{D}_{k}, Q \subset\right.$ $\left.Q_{\tau, k}^{j}\right\}$ with $\mathscr{I}=\bigcup_{N \in \mathbf{N}} \mathscr{I}_{N}$. From the proof of (6.22), we deduce that, for any $h \in L^{2}(\mathcal{X})$ with $\|h\|_{L^{2}(\mathcal{X})}=1$,

$$
\sum_{Q \in \mathscr{I}}\left|\left\langle D_{Q} D_{Q} f, h\right\rangle\right| \lesssim 2^{k}\left[\mu\left(Q_{\tau, k}^{j}\right)\right]^{1 / q}\|\widetilde{S}(h)\|_{L^{q^{\prime}}(\mathcal{X})} \lesssim 2^{k}\left[\mu\left(Q_{\tau, k}^{j}\right)\right]^{1 / q}<\infty,
$$

which further implies that, for any $N, M \in \mathbf{N}$ with $N>M$

$$
\left|\left\langle\sum_{Q \in \mathscr{I}_{N} \backslash \mathscr{I}_{M}} D_{Q} D_{Q} f, h\right\rangle\right| \leq \sum_{Q \in \mathscr{I}_{N} \backslash \mathscr{I}_{M}}\left|\left\langle D_{Q} D_{Q} f, h\right\rangle\right| \rightarrow 0 \quad \text { as } \quad M \rightarrow \infty .
$$

Thus, by the completion of $L^{2}(\mathcal{X})$ and $\left(L^{2}(\mathcal{X})\right)^{*}=L^{2}(\mathcal{X})$, where $\left(L^{2}(\mathcal{X})\right)^{*}$ denotes the dual space of $L^{2}(\mathcal{X})$, we obtain

$$
\sum_{\left\{Q \in \mathcal{D}_{k}, Q \subset Q_{\tau, k}^{j}\right\}} \int_{Q} D_{Q}(\cdot, y) D_{Q} f(y) d \mu(y)
$$

converges in $L^{2}(\mathcal{X})$, which, together with $D_{\ell-1}^{*}(x, y)=D_{\ell-1}(y, x)$ for any $\ell \in \mathbf{Z}$ and $(x, y) \in \mathcal{X} \times \mathcal{X}$, implies that

$$
\begin{aligned}
\left\langle b_{\tau, k}^{j}, \psi_{\widetilde{\beta}}^{\widetilde{k}}\right\rangle & =\frac{1}{\lambda_{\tau, k}^{j}} \sum_{\left\{Q \in \mathcal{D}_{k}, Q \subset Q_{\tau, k}^{j}\right\}} \int_{Q}\left\langle D_{Q}^{*}(\cdot, y), \psi_{\widetilde{\beta}}^{\widetilde{k}}\right\rangle D_{Q} f(y) d \mu(y) \\
& =\frac{1}{\lambda_{\tau, k}^{j}} \sum_{\ell=j}^{\infty} \sum_{\beta \in \mathscr{A}_{\ell}} \sum_{\left\{Q_{\beta}^{\ell} \in \mathcal{D}_{k}, Q_{\beta}^{\ell} \subset Q_{\tau, k}^{j}\right\}} \int_{Q_{\beta}^{\ell}}\left\langle D_{\ell-1}(y, \cdot), \psi_{\widetilde{\beta}}^{\widetilde{k}}\right\rangle D_{\ell} f(y) d \mu(y),
\end{aligned}
$$

where, for any $k \in \mathbf{Z}, D_{k}(\cdot, \cdot)$ is as in Remark 4.4(i) and $D_{k}^{*}(\cdot, \cdot)$ denotes the kernel of the adjoint operator $D_{k}^{*}$ of $D_{k}$ on $L^{2}(\mathcal{X})$.

On another hand, by the orthogonality of $\left\{\psi_{\widetilde{\beta}}^{\widetilde{k}}\right\}_{\widetilde{k} \in \mathbf{Z}, \widetilde{\beta} \in \mathscr{G}_{\tilde{k}}}$, we conclude that, for any $y \in \mathcal{X}$,

$$
\left\langle D_{\ell-1}(y, \cdot), \psi_{\widetilde{\beta}}^{\widetilde{k}}\right\rangle= \begin{cases}0, & \text { when } \ell-1 \neq \widetilde{k} \\ \psi_{\widetilde{\beta}}(y), & \text { when } \ell-1=\widetilde{k}\end{cases}
$$

which, combined with (6.26), implies that, if $\widetilde{k}<j-1$, then $\left\langle b_{\tau, k}^{j}, \psi_{\widetilde{\beta}}^{\widetilde{k}}\right\rangle=0$; if $\widetilde{k} \geq j-1$, then

$$
\left\langle b_{\tau, k}^{j}, \psi_{\widetilde{\beta}}^{\widetilde{k}}\right\rangle=\frac{1}{\lambda_{\tau, k}^{j}} \sum_{\beta \in \mathscr{A} \hat{k}+1} \sum_{\left\{Q_{\beta}^{\tilde{k}+1} \in \mathcal{D}_{k}, Q_{\beta}^{\widetilde{k}+1} \subset Q_{\tau, k}^{j}\right\}} \int_{Q_{\beta}^{\widetilde{k}+1}} \psi_{\widetilde{\beta}}^{\widetilde{k}}(y) D_{\widetilde{k}} f(y) d \mu(y) .
$$

From (6.19), (6.27), the disjointness of $\left\{Q_{\tau, k}^{j}\right\}_{\tau \in I_{j, k}}$ for any fixed $j, k \in \mathbf{Z}$, the disjointness of $\left\{\mathcal{D}_{k}\right\}_{k \in \mathbf{Z}}$, Lemma 4.1 (iii) and the orthogonality of $\left\{\psi_{\widetilde{\beta}}^{\widetilde{\kappa}}\right\}_{\widetilde{k} \in \mathbf{Z}, \widetilde{\beta} \in \mathscr{G}_{\widetilde{k}}}$, it 
follows that

$$
\begin{aligned}
\left\langle\widetilde{f}, \psi_{\widetilde{\beta}}^{\widetilde{k}}\right\rangle & =\sum_{k \in \mathbf{Z}} \sum_{j=-\infty}^{\widetilde{k}+1} \sum_{\tau \in I_{j, k}} \sum_{\beta \in \mathscr{A}_{\tilde{k}+1}} \sum_{\left\{Q_{\beta}^{\widetilde{k}+1} \in \mathcal{D}_{k}, Q_{\beta}^{\widetilde{k}+1} \subset Q_{\tau, k}^{j}\right\}} \int_{Q_{\beta}^{\tilde{k}+1}} \psi_{\widetilde{\beta}}^{\widetilde{k}}(y) D_{\widetilde{k}} f(y) d \mu(y) \\
& =\sum_{\beta \in \mathscr{A} \mathcal{A}_{\tilde{k}+1}} \int_{Q_{\beta}^{\tilde{k}+1}} \psi_{\widetilde{\beta}}^{\widetilde{k}}(y) D_{\widetilde{k}} f(y) d \mu(y) \\
& =\left\langle D_{\widetilde{k}} f, \psi_{\widetilde{\beta}}^{\widetilde{k}}\right\rangle=\sum_{\beta \in \mathscr{G}_{k}}\left\langle f, \psi_{\beta}^{k}\right\rangle\left\langle\psi_{\beta}^{k}, \psi_{\widetilde{\beta}}^{\widetilde{k}}\right\rangle=\left\langle f, \psi_{\widetilde{\beta}}^{\widetilde{k}}\right\rangle .
\end{aligned}
$$

This finishes the proof of (6.25) and hence of (6.20).

In the end, by (6.24), Proposition 6.12 and Theorem 6.8, we have

$$
\|f\|_{\stackrel{\circ}{\mathrm{at}}_{\mathrm{at}}(\mathcal{X})} \sim\|f\|_{H_{\mathrm{at}}^{\varphi, q}(\mathcal{X})} \sim\|f\|_{H_{\mathrm{mol}}^{\varphi, q, \epsilon}(\mathcal{X})} \lesssim \Lambda_{q}\left(\left\{\lambda_{\tau, k}^{j} b_{\tau, k}^{j}\right\}_{j, \tau, k}\right) \lesssim\|S(f)\|_{L^{\varphi}(\mathcal{X})} .
$$

This finishes the proof of $H^{\varphi}(\mathcal{X}) \subset \stackrel{\circ}{H}_{\mathrm{at}}^{\varphi, q}(\mathcal{X})$ and hence of Theorem 6.15.

6.4. Littlewood-Paley function characterizations of $\boldsymbol{H}^{\varphi}(\mathcal{X})$. In this subsection, we establish several characterizations of $H^{\varphi}(\mathcal{X})$, respectively, in terms of the Lusin area functions with apertures, the Littlewood-Paley $g$-functions and the Littlewood-Paley $g_{\lambda}^{*}$-functions, which is a generalization of the corresponding results on Hardy spaces $H^{p}(\mathcal{X})$ in [27, Theorem 5.10] [in this case, $\varphi$ is the same as in (4.2)].

Theorem 6.16. Let $\varphi$ be a growth function and $\varrho, \vartheta \in\left(\omega\left[\frac{q(\varphi)}{p}-1\right], \eta\right)$ with $q(\varphi)<\frac{p(\omega+\eta)}{\omega}$ as in (3.16). Assume that $\theta \in(0, \infty)$ and $\lambda \in\left(\omega\left[1+\frac{2 q(\varphi)}{p}\right], \infty\right)$. Then, for any $f \in\left(\dot{\mathcal{G}}_{0}^{\eta}(\varrho, \vartheta)\right)^{\prime}$, it holds true that

$$
\|f\|_{H^{\varphi}(\mathcal{X})} \sim\left\|\mathcal{S}_{\theta}(f)\right\|_{L^{\varphi}(\mathcal{X})} \sim\left\|g_{\lambda}^{*}(f)\right\|_{L^{\varphi}(\mathcal{X})} \sim\|g(f)\|_{L^{\varphi}(\mathcal{X})},
$$

provided that any one of (6.28) is finite, with all positive equivalence constants independent of $f$.

Proof. Let $f \in\left(\dot{\mathcal{G}}_{0}^{\eta}(\varrho, \vartheta)\right)^{\prime}$ with $\varrho, \vartheta \in\left(\omega\left[\frac{q(\varphi)}{p}-1\right], \eta\right)$. Let $S_{\theta}(f), g_{\lambda}^{*}(f)$ and $g(f)$ be defined, respectively, as in (6.1), (6.3) and (6.2) with $\left\{Q_{k}\right\}_{k \in \mathbf{Z}}$ being an exp-ATI, where $\theta \in(0, \infty)$ and $\lambda \in\left(\omega\left[1+\frac{2 q(\varphi)}{p}\right], \infty\right)$.

Let $(\varphi, q)$ be admissible as in Definition 5.2. We first show that $\left\|S_{\theta}(f)\right\|_{L^{\varphi}(\mathcal{X})} \sim$ $\|f\|_{H^{\varphi}(\mathcal{X})}$. From (6.18) and Theorem 6.15, we deduce that

$$
\left\|S_{\theta}(f)\right\|_{L^{\varphi}(\mathcal{X})} \lesssim\|f\|_{\stackrel{\circ}{\mathrm{at}}^{\varphi, q}(\mathcal{X})} \sim\|f\|_{H^{\varphi}(\mathcal{X})} .
$$

Conversely, without loss of generality, we may assume that $\left\|S_{\theta}(f)\right\|_{L^{\varphi}(\mathcal{X})}<\infty$. By the proof of $H^{\varphi}(\mathcal{X}) \subset \stackrel{\stackrel{H}{H}_{\mathrm{at}}^{\varphi, 2}}{(\mathcal{X})}$ in Theorem 6.15, we know that $f=\sum_{j=1}^{\infty} \lambda_{j} a_{j}$ in $\left(\stackrel{\mathcal{G}}{0}_{0}^{\eta}(\varrho, \vartheta)\right)^{\prime}$ with a sequence $\left\{a_{j}\right\}_{j=1}^{\infty}$ of adapted $(\varphi, q)_{A^{-a t o m s}}$ and numbers $\left\{\lambda_{j}\right\}_{j=1}^{\infty} \subset$ C such that

$$
\Lambda_{q}\left(\left\{\lambda_{j} a_{j}\right\}_{j \in \mathbf{N}}\right) \lesssim\left\|S_{\theta}(f)\right\|_{L^{\varphi}(\mathcal{X})},
$$

which, together with Theorem 6.15, further implies that

$$
\|f\|_{H^{\varphi}(\mathcal{X})}=\|S(f)\|_{L^{\varphi}(\mathcal{X})} \sim\|f\|_{\stackrel{\leftrightarrow}{\mathrm{at}}^{\varphi, q}(\mathcal{X})} \lesssim \Lambda_{q}\left(\left\{\lambda_{j} a_{j}\right\}_{j \in \mathbf{N}}\right) \lesssim\left\|S_{\theta}(f)\right\|_{L^{\varphi}(\mathcal{X})} .
$$

Therefore, we obtain $\|f\|_{H^{\varphi}(\mathcal{X})} \sim\left\|S_{\theta}(f)\right\|_{L^{\varphi}(\mathcal{X})}$. 
Then we show that $\|S(f)\|_{L^{\varphi}(\mathcal{X})} \sim\left\|g_{\lambda}^{*}(f)\right\|_{L^{\varphi}(\mathcal{X})}$. Notice that

$$
S(f) \lesssim g_{\lambda}^{*}(f) \lesssim \sum_{j=1}^{\infty} 2^{\frac{j(\omega-\lambda)}{2}} S_{2^{j}}(f)
$$

(see, for instance, the proof of [27, Theorem 5.10]), which, combined with (6.18) and Theorem 6.15, implies that

$$
\begin{aligned}
\|S(f)\|_{L^{\varphi}(\mathcal{X})}^{p} & \lesssim\left\|g_{\lambda}^{*}(f)\right\|_{L^{\varphi}(\mathcal{X})}^{p} \lesssim \sum_{j=1}^{\infty} 2^{\frac{j(\omega-\lambda)}{2} p}\left\|S_{2^{j}}(f)\right\|_{L^{\varphi}(\mathcal{X})}^{p} \\
& \lesssim \sum_{j=1}^{\infty} 2^{\frac{j(\omega-\lambda)}{2} p}\|f\|_{H_{\mathrm{at}}^{\varphi, q}(\mathcal{X})}^{p} \lesssim\|f\|_{H_{\mathrm{at}}^{\varphi, q}(\mathcal{X})}^{p} \sim\|S(f)\|_{L^{\varphi}(\mathcal{X})}^{p} .
\end{aligned}
$$

This shows that $\|S(f)\|_{L^{\varphi}(\mathcal{X})}^{p} \sim\left\|g_{\lambda}^{*}(f)\right\|_{L^{\varphi}(\mathcal{X})}^{p}$.

Now, we prove $\|g(f)\|_{L^{\varphi}(\mathcal{X})} \sim\|f\|_{H^{\varphi}(\mathcal{X})}$. Following the proof of (6.18), we easily have

$$
\|g(f)\|_{L^{\varphi}(\mathcal{X})} \lesssim\|f\|_{H_{\mathrm{at}}^{\varphi, q}(\mathcal{X})} \sim\|f\|_{H^{\varphi}(\mathcal{X})} .
$$

To finish the proof of Theorem 6.16, we still need to show that $\|f\|_{H^{\varphi}(\mathcal{X})} \lesssim\|g(f)\|_{L^{\varphi}(\mathcal{X})}$. Indeed, by the proof of [27, Theorem 5.10] with the same notation as therein, via choosing $r$ and $\widetilde{\varrho}$ such that $r \in\left(\frac{\omega}{\omega+\widetilde{\varrho}}, \frac{p}{q(\varphi)}\right)$, we conclude that, for any $x \in \mathcal{X}$,

$$
[S(f)(x)]^{2} \lesssim \sum_{k^{\prime} \in \mathbf{Z}}\left[M\left(\sum_{\alpha^{\prime} \in \mathscr{A}_{k^{\prime}}} \sum_{m^{\prime}=1}^{N\left(k^{\prime}, \alpha^{\prime}\right)}\left|Q_{k^{\prime}} f\left(y_{\alpha^{\prime}}^{k^{\prime}, m^{\prime}}\right)\right|^{r} \mathbf{1}_{Q_{\alpha^{\prime}}^{k^{\prime}, m^{\prime}}}\right)(x)\right]^{\frac{2}{r}}
$$

which, together with Theorem 6.6, further implies that

$$
\begin{aligned}
\|f\|_{H^{\varphi}(\mathcal{X})} & =\left\|[S(f)]^{r}\right\|_{L^{\tilde{\varphi}}(\mathcal{X})}^{\frac{1}{r}} \\
& \left.\lesssim\left\|\left\{\sum_{k^{\prime} \in \mathbf{Z}}\left[M\left(\sum_{\alpha^{\prime} \in \mathscr{A}_{k^{\prime}}} \sum_{m^{\prime}=1}^{N\left(k^{\prime}, \alpha^{\prime}\right)}\left|Q_{k^{\prime}} f\left(y_{\alpha^{\prime}}^{k^{\prime}, m^{\prime}}\right)\right|^{r} \mathbf{1}_{Q_{\alpha^{\prime}}^{k^{\prime}, m^{\prime}}}\right)\right]^{\frac{2}{r}}\right\}\right\|_{L^{\tilde{\varphi}}(\mathcal{X})}^{\frac{r}{2}}\right|^{\frac{1}{r}} \\
& \lesssim\left\|\left\{\sum_{k \in \mathbf{Z}}\left[\sum_{\alpha^{\prime} \in \mathscr{A}_{k^{\prime}}} \sum_{m^{\prime}=1}^{N\left(k^{\prime}, \alpha^{\prime}\right)}\left|Q_{k^{\prime}} f\left(y_{\alpha^{\prime}}^{k^{\prime}, m^{\prime}}\right)\right|^{r} \mathbf{1}_{Q_{\alpha^{\prime}}^{k^{\prime}, m^{\prime}}}\right]^{\frac{2}{r}}\right\}^{\frac{r}{2}}\right\|_{L_{L^{\tilde{\varphi}}(\mathcal{X})}^{\frac{1}{r}}} \\
& \left.\sim \| \sum_{k^{\prime} \in \mathbf{Z}} \sum_{\alpha^{\prime} \in \mathscr{A}_{k^{\prime}}} \sum_{m^{\prime}=1}^{N\left(k^{\prime}, \alpha^{\prime}\right)}\left|Q_{k^{\prime}} f\left(y_{\alpha^{\prime}}^{k^{\prime}, m^{\prime}}\right)\right|^{2} \mathbf{1}_{Q_{\alpha^{\prime}}^{k^{\prime}, m^{\prime}}}\right]^{\frac{1}{2}} \|_{L^{\varphi}(\mathcal{X})},
\end{aligned}
$$

where $\widetilde{\varphi}(x, t):=\varphi\left(x, t^{1 / r}\right)$ for any $(x, t) \in \mathcal{X} \times[0, \infty)$ is of uniformly lower type $p / r \in(q(\varphi), \infty)$. From this and the arbitrariness of $y_{\alpha^{\prime}}^{k^{\prime}, m^{\prime}}$, it follows that

$$
\|f\|_{H \varphi(\mathcal{X})} \lesssim\left\|\left[\sum_{k \in \mathbf{Z}} \sum_{\alpha^{\prime} \in \mathscr{A}_{k}} \sum_{m^{\prime}=1}^{N\left(k^{\prime}, \alpha^{\prime}\right)} \inf _{z \in Q_{k^{\prime}}\left(y_{\alpha^{\prime}}^{k^{\prime}, m^{\prime}}\right)}\left|Q_{k^{\prime}} f(z)\right|^{2} \mathbf{1}_{Q_{\alpha^{\prime}}^{k^{\prime}, m^{\prime}}}\right]^{\frac{1}{2}}\right\|_{L^{\varphi}(\mathcal{X})} \lesssim\|g(f)\|_{L^{\varphi}(\mathcal{X})}
$$

which completes the proof of $\|f\|_{H^{\varphi}(\mathcal{X})} \lesssim\|g(f)\|_{L^{\varphi}(\mathcal{X})}$ and hence of Theorem 6.16. 


\section{Finite atomic characterizations}

In this section, we obtain a finite atomic characterization of $H^{\varphi}(\mathcal{X})$ and also give an improved version in case $q<\infty$.

7.1. Finite atomic characterizations with uniformly locally dominated convergence conditions. In this subsection, we obtain a finite atomic characterization of $H^{\varphi}(\mathcal{X})$ with uniformly locally dominated convergence conditions.

First, we need to introduce the following uniformly locally dominated convergence condition on $\varphi$, which is a generalization of the corresponding Euclidean case in [35] to any space of homogeneous type.

Definition 7.1. A growth function $\varphi$ is said to satisfy the uniformly locally dominated convergence condition if the following holds true: Let $K \subset \mathcal{X}$ be a bounded set and $f$ a measurable function on $\mathcal{X}$ such that $\lim _{m \rightarrow \infty} f_{m}(x)=f(x)$ for almost every $x \in \mathcal{X}$. If there exists a non-negative measurable function $g$ such that $\left|f_{m}(x)\right| \leq$ $g(x)$ for almost every $x \in \mathcal{X}$ and

$$
\sup _{t \in(0, \infty)} \int_{K} g(x) \frac{\varphi(x, t)}{\int_{K} \varphi(y, t) d \mu(y)} d \mu(x)<\infty
$$

then

$$
\lim _{m \rightarrow \infty} \sup _{t \in(0, \infty)} \int_{K}\left|f_{m}(x)-f(x)\right| \frac{\varphi(x, t)}{\int_{K} \varphi(y, t) d \mu(y)} d \mu(x)=0 .
$$

We first recall some notions and notation from [35, Section 2.4]. Recall that a quasi-Banach space $\mathcal{B}$ is a complete vector space equipped with a quasi-norm $\|\cdot\|_{\mathcal{B}}$ which is nonnegative, non-degenerate (namely, $\|f\|_{\mathcal{B}}=0$ if and only if $f=0$ ) and homogeneous, and satisfies the quasi-triangle inequality, namely, there exists a positive constant $\kappa \in[1, \infty)$ such that, for any $f, g \in \mathcal{B},\|f+g\|_{\mathcal{B}} \leq \kappa\left(\|f\|_{\mathcal{B}}+\|g\|_{\mathcal{B}}\right)$.

Let $\gamma \in(0,1]$ and $m \in \mathbf{N}$. A quasi-Banach space $\mathcal{B}_{\gamma}$ with the quasi-norm $\|\cdot\|_{\mathcal{B}_{\gamma}}$ is called a $\gamma$-quasi-Banach space if there exists a positive constant $\kappa \in[1, \infty)$ such that, for any $m \in \mathbf{N}$ and $\left\{f_{j}\right\}_{j=1}^{m} \subset \mathcal{B}_{\gamma}$,

$$
\left\|\sum_{j=1}^{m} f_{j}\right\|_{\mathcal{B}_{\gamma}}^{\gamma} \leq \kappa \sum_{j=1}^{m}\left\|f_{j}\right\|_{\mathcal{B}_{\gamma}}^{\gamma} .
$$

Similarly to the Euclidean case, we know that, when $\varphi$ is of uniformly lower type $p \in(0,1], H^{\varphi}(\mathcal{X})$ and $L^{\varphi}(\mathcal{X})$ are $p$-quasi-Banach spaces.

For every given $\gamma$-quasi-Banach space $\mathcal{B}_{\gamma}$ with $\gamma \in(0,1]$ and a linear space $\mathcal{Y}$, an operator $T$ from $\mathcal{Y}$ to $\mathcal{B}_{\gamma}$ is said to be $\mathcal{B}_{\gamma}$-sublinear if there exists a positive constant $\tilde{\kappa} \in[1, \infty)$ such that

(i) for any $f, g \in \mathcal{Y},\|T(f)-T(g)\|_{\mathcal{B}_{\gamma}} \leq \widetilde{\kappa}\|T(f-g)\|_{\mathcal{B}_{\gamma}}$;

(ii) for any $m \in \mathbf{N},\left\{\lambda_{j}\right\}_{j=1}^{m} \subset \mathbf{C}$ and $\left\{f_{j}\right\}_{j=1}^{m} \subset \mathcal{Y}$,

$$
\left\|T\left(\sum_{j=1}^{m} \lambda_{j} f_{j}\right)\right\|_{\mathcal{B}_{\gamma}}^{\gamma} \leq \tilde{\kappa} \sum_{j=1}^{m}\left|\lambda_{j}\right|^{\gamma}\left\|T\left(f_{j}\right)\right\|_{\mathcal{B}_{\gamma}}^{\gamma} .
$$

The main result of this section is stated as follows. Let $\mathrm{UC}(\mathcal{X})$ be the set of all uniformly continuous functions on $\mathcal{X}$. 
Theorem 7.2. Let $\varphi$ be a growth function as in Definition $2.5,(\varphi, q)$ be admissible as in Definition 5.2 and $\varphi$ satisfy the uniformly locally dominated convergence condition as in Definition 7.1. Then

(i) If $q \in(q(\varphi), \infty)$, then $\|\cdot\|_{H_{\mathrm{fin}}^{\varphi, q}(\mathcal{X})}$ and $\|\cdot\|_{H^{\varphi}(\mathcal{X})}$ are equivalent quasi-norms on $H_{\text {fin }}^{\varphi, q}(\mathcal{X})$;

(ii) $\|\cdot\|_{H_{\mathrm{fin}}^{\varphi, \infty}(\mathcal{X})}$ and $\|\cdot\|_{H^{\varphi}(\mathcal{X})}$ are equivalent quasi-norms on $H_{\mathrm{fin}}^{\varphi, \infty}(\mathcal{X}) \cap \mathrm{UC}(\mathcal{X})$;

(iii) $H_{\mathrm{fin}}^{\varphi, \infty}(\mathcal{X}) \cap \mathrm{UC}(\mathcal{X})$ is a dense subspace of $H^{\varphi}(\mathcal{X})$.

Proof. We first show (i). Let $q \in(q(\varphi), \infty)$. By Theorems 6.15 and 5.4, and Proposition 6.12 , we know that $H^{\varphi}(\mathcal{X})$ and $H^{*, \varphi}(\mathcal{X})$ coincide with equivalent quasinorms and, for any $f \in H^{\varphi}(\mathcal{X})$,

$$
\|f\|_{H^{\varphi}(\mathcal{X})} \sim\|f\|_{H_{\mathrm{at}}^{\varphi}(\mathcal{X})} \sim\|f\|_{H_{\mathrm{at}}^{\varphi, q}(\mathcal{X})} \sim\|f\|_{H^{*, \varphi}(\mathcal{X})} .
$$

Thus, to show (i), it suffices to prove that, for any $f \in H_{\text {fin }}^{\varphi, q}(\mathcal{X})$,

$$
\|f\|_{H_{\text {fin }}^{\varphi, q}(\mathcal{X})} \lesssim\|f\|_{H^{*, \varphi}(\mathcal{X})}
$$

By the homogeneity of the quasi-norm of $H^{*, \varphi}(\mathcal{X})$, without loss of generality, we may assume that $\|f\|_{H^{*, \varphi}(\mathcal{X})}=1$.

Obviously, for any $f \in H_{\text {fin }}^{\varphi, q}(\mathcal{X}), f \in L_{\varphi(\cdot, 1)}^{q}(\mathcal{X}) \cap H^{*, \varphi}(\mathcal{X})$ and $\int_{\mathcal{X}} f(x) d \mu(x)=0$. By the atomic characterization in Part II of the proof of Theorem 5.4, namely, the proof of $H^{*, \varphi}(\mathcal{X}) \subset H_{\mathrm{at}}^{\varphi, q}(\mathcal{X})$ (with the same notation as therein),

$$
f=\sum_{j \in \mathbf{Z}} \sum_{k \in I_{j}} \lambda_{k}^{j} a_{k}^{j}=\sum_{j \in \mathbf{Z}} \sum_{k \in I_{j}} h_{k}^{j}=\sum_{j \in \mathbf{Z}} h_{j}
$$

converges both in $\left(\mathcal{G}_{0}^{\eta}(\varrho, \vartheta)\right)^{\prime}$ and almost everywhere on $\mathcal{X}$. Here and hereafter, $h_{j}$, $h_{k}^{j}, \lambda_{k}^{j}$ and $a_{k}^{j}$ are as in (5.13) and (5.15).

From $f \in H_{\text {fin }}^{\varphi, q}(\mathcal{X})$, we deduce that there exists a ball $B\left(x_{1}, R\right)$ with $x_{1} \in \mathcal{X}$ and $R \in(0, \infty)$ such that supp $f \subset B\left(x_{1}, R\right)$. In the remainder of this proof, let $\widetilde{B}:=B\left(x_{1}, 16 A_{0}^{4} R\right)$. Now, we claim that, for any $x \in(\widetilde{B})^{\complement}$,

$$
f^{\star}(x) \leq c_{1}\left\|\mathbf{1}_{\widetilde{B}}\right\|_{L^{\varphi}(\mathcal{X})}^{-1}
$$

for some fixed positive constant $c_{1}$ independent of $f$ and $x$, where $f^{\star}$ is as in (5.4). Suppose that $\varphi \in\left(\mathcal{G}_{0}^{\eta}(\varrho, \vartheta)\right)^{\prime}$ with $\|\varphi\|_{\mathcal{G}(x, r, \varrho, \vartheta)} \leq 1$ for some $r \in(0, \infty)$. By the proof of $[27,(7.1)]$, we know that, for any $y \in B\left(x_{1}, d\left(x, x_{1}\right)\right)$,

$$
|\langle f, \varphi\rangle| \lesssim f^{*}(y)
$$

where $f^{*}$ is as in (4.3), and hence, by (3.19), for any $x \in(\widetilde{B})^{\complement}$,

$$
\begin{aligned}
f^{\star}(x) & \lesssim \inf _{y \in B\left(x_{1}, R\right)} f^{*}(y) \lesssim\left\|\mathbf{1}_{B\left(x_{1}, R\right)}\right\|_{L^{\varphi}(\mathcal{X})}^{-1}\left\|f^{*}\right\|_{L^{\varphi}(\mathcal{X})} \sim\left\|\mathbf{1}_{\widetilde{B}}\right\|_{L^{\varphi}(\mathcal{X})}^{-1}\|f\|_{H^{*, \varphi}(\mathcal{X})} \\
& =: c_{1}\left\|\mathbf{1}_{\widetilde{B}}\right\|_{L^{\varphi}(\mathcal{X})}^{-1}
\end{aligned}
$$

for some fixed positive constant $c_{1}$ independent of $f$ and $x$, which completes the proof of the above claim.

Moreover, let

$$
\widetilde{j}:=\max \left\{j \in \mathbf{Z}: 2^{j} \leq c_{1}\left\|\mathbf{1}_{\widetilde{B}}\right\|_{L^{\varphi}(\mathcal{X})}^{-1}\right\} .
$$


Then, by the atomic characterization in Part II of the proof of Theorem 5.4, namely, the proof of $H^{*, \varphi}(\mathcal{X}) \subset H_{\mathrm{at}}^{\varphi, q}(\mathcal{X})$, we know that

$$
h:=\sum_{j \leq \tilde{j}} \sum_{k \in I_{j}} h_{k}^{j} \quad \text { and } \quad \ell:=\sum_{j>\widetilde{j}} \sum_{k \in I_{j}} h_{k}^{j}
$$

converge both in $\left(\mathcal{G}_{0}^{\eta}(\varrho, \vartheta)\right)^{\prime}$ and almost everywhere on $\mathcal{X}$, where $\left\{h_{k}^{j}\right\}_{j \in \mathbf{Z}, k \in I_{j}}$ are the same as in (5.13) and, indeed, $h_{k}^{j}$ for any $i \in \mathbf{Z}$ and $k \in I_{j}$ is a multiple of a $(\varphi, \infty)$-atom as in Definition 5.2. Thus, $f=h+\ell$ and, by the above claim (7.2), we conclude that, if $j>\widetilde{j}$, then

$$
\Omega^{j}:=\left\{x \in \mathcal{X}: f^{\star}(x)>2^{j}\right\} \subset \widetilde{B},
$$

which, combined with supp $f \subset B\left(x_{1}, R\right)$, further implies that supp $h \subset \widetilde{B}$. Now, we show that $h$ is a multiple of a $(\varphi, \infty)$-atom as in Definition 5.2. Indeed, we recall some results from Part II of the proof of Theorem 5.4, namely, the proof of $H^{*, \varphi}(\mathcal{X}) \subset H_{\text {at }}^{\varphi, q}(\mathcal{X})$, that

$$
\begin{aligned}
& \left\|h_{k}^{j}\right\|_{L^{\infty}(\mathcal{X})} \lesssim 2^{j}, \quad \operatorname{supp} h_{k}^{j} \subset B_{k}^{j}:=B\left(x_{k}^{j}, 16 A_{0}^{4} r_{k}^{j}\right) \subset \Omega^{j} \\
& \text { and } \sum_{j \in I_{k}} \mathbf{1}_{B\left(x_{k}^{j}, 16 A_{0}^{4} r_{k}^{j}\right)} \lesssim 1 .
\end{aligned}
$$

Therefore, we have

$$
\|h\|_{L^{\infty}(\mathcal{X})} \leq \sum_{j \leq \widetilde{j}}\left\|h_{j}\right\|_{L^{\infty}(\mathcal{X})} \lesssim \sum_{j \leq \widetilde{j}} 2^{j} \lesssim\left\|\mathbf{1}_{\widetilde{B}}\right\|_{L^{\varphi}(\mathcal{X})}^{-1} .
$$

Thus, to prove that $h$ is a multiple of a $(\varphi, \infty)$-atom, it suffices to show that $\int_{\mathcal{X}} h(x) d \mu(x)=0$. To this end, we first need to prove that $f$ is a multiple of a classical $(1, \widetilde{q})$-atom as in $\left[10\right.$, p. 591] for some $\widetilde{q} \in\left(1, \frac{q}{q(\varphi)}\right)$. Let $\widetilde{q} \in\left(1, \frac{q}{q(\varphi)}\right)$. Then $\varphi \in \mathbf{A}_{q / \widetilde{q}}(\mathcal{X})$ and hence

$$
\begin{aligned}
& {\left[\frac{1}{V\left(x_{1}, R\right)} \int_{B\left(x_{1}, R\right)}|f(x)|^{\widetilde{q}} d \mu(x)\right]^{1 / \widetilde{q}}} \\
& \leq\left[\frac{1}{\varphi\left(B\left(x_{1}, R\right), 1\right)} \int_{B\left(x_{1}, R\right)}|f(x)|^{q} \varphi(x, 1) d \mu(x)\right]^{1 / q}<\infty
\end{aligned}
$$

by Lemma 2.6(iii) when $q \in(q(\varphi), \infty)$. From this and $\int_{\mathcal{X}} f(x) d \mu(x)=0$, it follows that $f$ is a multiple of a classical $(1, \widetilde{q})$-atom and hence $f^{*} \in L^{1}(\mathcal{X})$.

Moreover, by (7.4), for any $x \in \mathcal{X}$, we have

$$
\begin{aligned}
& \sum_{j>\widetilde{j}} \sum_{k \in I_{j}}\left|h_{k}^{j}(x)\right| \\
& \lesssim \sum_{j>\widetilde{j}} 2^{j} \sum_{k \in I_{j}} \mathbf{1}_{B_{k}^{j}}(x) \lesssim \sum_{j>\widetilde{j}} 2^{j} \mathbf{1}_{\Omega^{j}}(x) \sim \sum_{j>\widetilde{j}} 2^{j} \sum_{m=j}^{\infty} \mathbf{1}_{\Omega^{m} \backslash \Omega^{m+1}}(x) \\
& \sim \sum_{m=\tilde{j}+1}^{\infty} \sum_{j=\tilde{j}+1}^{m} 2^{j} \mathbf{1}_{\Omega^{m} \backslash \Omega^{m+1}}(x) \sim \sum_{m=\tilde{j}+1}^{\infty} 2^{m} \mathbf{1}_{\Omega^{m} \backslash \Omega^{m+1}}(x) \\
& \sim f^{*}(x) \sum_{m=\tilde{j}+1}^{\infty} \mathbf{1}_{\Omega^{m} \backslash \Omega^{m+1}}(x) \sim f^{*}(x) \mathbf{1}_{\Omega^{\tilde{j}+1}}(x) \lesssim f^{*}(x) \mathbf{1}_{\Omega^{j}}(x) \in L^{1}(\mathcal{X}),
\end{aligned}
$$


which, together with the Lebesgue dominated convergence theorem, implies that

$$
\int_{\mathcal{X}} \ell(x) d \mu(x)=\sum_{j>\tilde{j}} \sum_{k \in I_{j}} \int_{\mathcal{X}} h_{k}^{j}(x) d \mu(x)=0 .
$$

Moreover, by this and $\int_{\mathcal{X}} f(x) d \mu(x)=0$, we obtain

$$
\int_{\mathcal{X}} h(x) d \mu(x)=\int_{\mathcal{X}} f(x) d \mu(x)-\int_{\mathcal{X}} \ell(x) d \mu(x)=0,
$$

which further implies that $h$ is a multiple of a $(\varphi, \infty)$-atom as in Definition 5.2.

Now, we deal with $\ell$. For any $N \in \mathbf{N}$, let

$$
F_{N}:=\left\{(k, j): j \in \mathbf{Z}, k \in I_{j}, j>\widetilde{j},|k|+|j| \leq N\right\} \quad \text { and } \quad \ell_{N}:=\sum_{(k, j) \in F_{N}} h_{k}^{j} .
$$

Then $\left\|\ell_{N}\right\|_{H_{\text {fin }}^{\varphi, q}(\mathcal{X})} \leq \Lambda\left(\left\{h_{k}^{j}\right\}_{(k, j) \in F_{N}}\right) \lesssim 1$. We then claim that, for any $\epsilon \in(0, \infty)$, there exists $N \in \mathbf{N}$ such that $\varepsilon^{-1}\left(\ell-\ell_{N}\right)$ is a $(\varphi, q)$-atom supported in the ball $\widetilde{B}$ and $\left\|\ell-\ell_{N}\right\|_{H_{\text {fin }}^{\varphi, q}(\mathcal{X})}<\varepsilon$. Indeed, observe that, for any $j>\widetilde{j}$,

$$
\operatorname{supp}\left(\ell-\ell_{N}\right) \subset \Omega^{j} \subset \widetilde{B} \text { and } \int_{\mathcal{X}}\left[\ell(x)-\ell_{N}(x)\right] d \mu(x)=0 .
$$

To prove the above claim, it suffices to show that $\lim _{N \rightarrow \infty}\left\|\ell-\ell_{N}\right\|_{L_{\varphi}^{q}(\widetilde{B})}=0$. To this end, by $f \in L_{\varphi}^{q}(\widetilde{B}), f^{*} \lesssim M(f)$ (see, for instance, [21, Proposition 3.9]) and Lemma 2.6(v), we conclude that $f^{*} \in L_{\varphi}^{q}(\widetilde{B})$, which, combined with $(7.5)$ and the uniformly locally dominated convergence condition as in Definition 7.1, further implies that $\lim _{N \rightarrow \infty}\left\|\ell-\ell_{N}\right\|_{L_{\varphi}^{q}(\widetilde{B})}=0$. This shows the above claim.

Furthermore, for any $\varepsilon \in(0, \infty)$, we choose $N \in \mathbf{N}$ such that $\left\|\ell_{N}-\ell\right\|_{H_{\text {fin }}^{\varphi, q}(\mathcal{X})}<\epsilon$. Thus, $f=h+\ell_{N}+\left(\ell-\ell_{N}\right)$ is a finite linear combination of both $(\varphi, \infty)$-atoms and $(\varphi, q)$-atoms and

$$
\|f\|_{H_{\mathrm{fin}}^{\varphi, q}(\mathcal{X})} \lesssim\|h\|_{H_{\mathrm{fin}}^{\varphi, q}(\mathcal{X})}+\left\|\ell_{N}\right\|_{H_{\mathrm{fin}}^{\varphi, q}(\mathcal{X})}+\left\|\ell-\ell_{N}\right\|_{H_{\mathrm{fin}}^{\varphi, q}(\mathcal{X})} \lesssim 1,
$$

which completes the proof of (i).

To show (ii), by Theorems 6.15 and 5.4, and Proposition 6.12, we know that $H^{\varphi}(\mathcal{X})$ and $H_{\mathrm{at}}^{\varphi, \infty}(\mathcal{X})$ coincide with equivalent quasi-norms and, for any $f \in H^{\varphi}(\mathcal{X})$,

$$
\|f\|_{H^{\varphi}(\mathcal{X})} \sim\|f\|_{H_{\mathrm{at}}^{\varphi, q}(\mathcal{X})} \sim\|f\|_{H_{\mathrm{at}}^{\varphi, q}(\mathcal{X})} \sim\|f\|_{H_{\mathrm{at}}^{\varphi, \infty}(\mathcal{X})} .
$$

Thus, to show (ii), it suffices to prove that, for any $f \in H_{\text {fin }}^{\varphi, \infty}(\mathcal{X}) \cap \operatorname{UC}(\mathcal{X})$,

$$
\|f\|_{H_{\text {fin }}^{\varphi, \infty}(\mathcal{X})} \lesssim\|f\|_{H_{\mathrm{at}}^{\varphi, \infty}(\mathcal{X})} .
$$

By the homogeneity of the quasi-norm of $H^{*, \varphi}(\mathcal{X})$, without loss of generality, we may assume that $\|f\|_{H_{\mathrm{at}}^{\varphi, \infty}(\mathcal{X})}=1$. We use some arguments from the proof of $[27$, Theorem 7.1].

Since $f \in \underset{\text { fin }}{\varphi, \infty}(\mathcal{X}) \cap \mathrm{UC}(\mathcal{X})$, it follows that $f$ is bounded. Thus, there exists a positive integer $\underset{j}{\widetilde{j}}>\widetilde{j}$ such that $\Omega^{j}=\emptyset$ for any integer $j>\widetilde{\tilde{j}}$. Consequently,

$$
\ell=\sum_{\widetilde{j}<j \leq \widetilde{j}} \sum_{k} h_{k}^{j}
$$


where $\ell$ and $\left\{h_{k}^{j}\right\}_{j \in \mathbf{Z}, k \in I_{j}}$ are the same as in the proof of (i). By $f \in \operatorname{UC}(\mathcal{X})$, we obtain, for any $\epsilon \in(0, \infty)$, there exists $\sigma \in(0, \infty)$ such that, if $d(x, y) \leq \sigma$, then $|f(x)-f(y)|<\epsilon$. Write $\ell:=\ell_{1}^{\sigma}+\ell_{2}^{\sigma}$ with

$$
\ell_{1}^{\sigma}:=\sum_{(j, k) \in G_{1}} h_{k}^{j} \quad \text { and } \quad \ell_{2}^{\sigma}:=\sum_{(j, k) \in G_{2}} h_{k}^{j},
$$

where

$$
G_{1}:=\left\{(k, j): j \in \mathbf{Z}, k \in I_{j}, 12 A_{0}^{3} r_{k}^{j} \geq \sigma, \widetilde{j}<j \leq \widetilde{\widetilde{j}}\right\}
$$

and

$$
G_{2}:=\left\{(k, j): j \in \mathbf{Z}, k \in I_{j}, 12 A_{0}^{3} r_{k}^{j}<\sigma, \widetilde{j}<j \leq \widetilde{\widetilde{j}}\right\}
$$

Notice that $\Omega^{j}$ is bounded for any $\left.j \in \widetilde{(j}, \widetilde{j}\right]$, which, together with [27, Proposition 4.4(vi)], implies that $\ell_{1}^{\sigma}$ is a finite linear combination of $(\varphi, \infty)$-atoms as in Definition 5.2 and $\left\|\ell_{1}^{\sigma}\right\|_{H_{\text {fin }}^{\varphi, \infty}(\mathcal{X})} \leq \Lambda_{\infty}\left(\left\{h_{k}^{j}\right\}_{(k, j) \in G_{1}}\right) \lesssim 1$.

For $\ell_{2}^{\sigma}$, from the proof of [27, Theorem 7.1], we deduce that $\operatorname{supp} \ell_{2}^{\sigma} \subset \widetilde{B}$, $\int_{\mathcal{X}} \ell_{2}^{\sigma}(x) d \mu(x)=0$ and

$$
\left\|\ell_{2}^{\sigma}\right\|_{L^{\infty}(\mathcal{X})} \lesssim \epsilon
$$

Thus, $\ell_{2}^{\sigma}$ is a multiple of a $(\varphi, \infty)$-atom as in Definition 5.2. This proves that $\left\|\ell_{2}^{\sigma}\right\|_{H_{\text {fin }}^{\varphi, \infty}(\mathcal{X})} \lesssim 1$ and hence

$$
\|f\|_{H_{\text {fin }}^{\varphi, \infty}(\mathcal{X})} \lesssim\|h\|_{H_{\text {fin }}^{\varphi, q}(\mathcal{X})}+\left\|\ell_{1}^{\sigma}\right\|_{H_{\text {fin }}^{\varphi, \infty}(\mathcal{X})}+\left\|\ell_{2}^{\sigma}\right\|_{H_{\text {fin }}^{\varphi, q}(\mathcal{X})} \lesssim 1
$$

which completes the proof of (ii).

Finally, to prove (iii), let $q \in(q(\varphi), \infty)$ and $a$ be any $(\varphi, \infty)$-atom supported in $B(z, r)$ with $z \in \mathcal{X}$ and $r \in(0, \infty)$. By (7.6) and the proof of [27, Theorem 7.1(iii)] (with the same notation as therein), we know that, to show (iii), it suffices to show that

$$
\lim _{k \rightarrow \infty}\left\|S_{k} a-a\right\|_{L_{\varphi}^{q}\left(B\left(z, 2 A_{0} r\right)\right)}=0 .
$$

Let $\widetilde{q} \in(1, \infty)$ satisfy $\frac{\widetilde{q}}{q}>[r(\varphi)]^{\prime}>1$. Then $\left(\frac{\widetilde{q}}{q}\right)^{\prime}<r(\varphi)$ and hence $\varphi \in \mathbf{R H}_{\left(\frac{\widetilde{q}}{q}\right)^{\prime}}(\mathcal{X})$. By this and the Hölder inequality, we conclude that, for any $t \in(0, \infty)$,

$$
\begin{aligned}
& \frac{1}{\varphi\left(B\left(z, 2 A_{0} r\right), t\right)} \int_{B\left(z, 2 A_{0} r\right)}\left|S_{k} a(x)-a(x)\right|^{q} \varphi(x, t) d \mu(x) \\
\leq & \frac{1}{\varphi\left(B\left(z, 2 A_{0} r\right), t\right)}\left[\int_{B\left(z, 2 A_{0} r\right)}\left|S_{k} a(x)-a(x)\right|^{\widetilde{q}} d \mu(x)\right]^{\frac{q}{q}} \\
& \cdot\left\{\int_{B\left(z, 2 A_{0} r\right)}[\varphi(x, t)]^{\left(\frac{\tilde{q}}{q}\right)^{\prime}} d \mu(x)\right\}^{1 /\left(\frac{\tilde{q}}{q}\right)^{\prime}} \\
\lesssim & \frac{1}{\varphi\left(B\left(z, 2 A_{0} r\right), t\right)}\left\|S_{k} a-a\right\|_{L^{\widetilde{q}}\left(B\left(z, 2 A_{0} r\right)\right)}^{q}\left[V\left(z, 2 A_{0} r\right)\right]^{1 /\left(\frac{\tilde{q}}{q}\right)^{\prime}-1} \varphi\left(B\left(z, 2 A_{0} r\right), t\right) \\
\sim & {\left[V\left(z, 2 A_{0} r\right)\right]^{-\frac{q}{q}}\left\|S_{k} a-a\right\|_{L^{\widetilde{q}}\left(B\left(z, 2 A_{0} r\right)\right)}^{q}, }
\end{aligned}
$$

which, combined with [26, Proposition 2.7(iv)], further implies that, as $k \rightarrow \infty$,

$$
\left\|S_{k} a-a\right\|_{L_{\varphi}^{q}\left(B\left(z, 2 A_{0} r\right)\right)} \lesssim\left[V\left(z, 2 A_{0} r\right)\right]^{-\frac{q}{\tilde{q}}}\left\|S_{k} a-a\right\|_{L^{\widetilde{q}}\left(B\left(z, 2 A_{0} r\right)\right)}^{q} \rightarrow 0 .
$$

This finishes the proof of (iii) and hence of Theorem 7.2. 
As an application, we formulate some criteria for the boundedness of quasiBanach valued sublinear operators on $H^{\varphi}(\mathcal{X})$, whose proofs are similar to that of [35, Theorem 3.5]; the details are omitted.

Theorem 7.3. Let $\varphi$ be a growth function satisfying the uniformly locally dominated convergence condition as in Definition 7.1. Let $(\varphi, q)$ be admissible as in Definition 5.2 and $\varphi$ of uniformly upper type $\gamma \in(0,1]$. Suppose that $\mathcal{B}_{\gamma}$ is a $\gamma$ quasi-Banach space and one of the following statements hold true:

(i) $q \in(q(\varphi), \infty)$ and $T: H_{\text {fin }}^{\varphi, q}(\mathcal{X}) \rightarrow \mathcal{B}_{\gamma}$ is a $\mathcal{B}_{\gamma}$-sublinear operator such that

$$
\sup \left\{\|T a\|_{\mathcal{B}_{\gamma}}: a \text { is a }(\varphi, q)-\operatorname{atom}\right\}<\infty ;
$$

(ii) $T$ is a $\mathcal{B}_{\gamma}$-sublinear operator defined on continuous $(\varphi, \infty)$-atoms such that

$$
\sup \left\{\|T a\|_{\mathcal{B}_{\gamma}}: a \text { is a continuous }(\varphi, \infty)-\operatorname{atom}\right\}<\infty .
$$

Then $T$ can be uniquely extended to a bounded $\mathcal{B}_{\gamma}$-sublinear operator from $H^{\varphi}(\mathcal{X})$ into $\mathcal{B}_{\gamma}$.

7.2. Finite atomic characterizations without having recourse to uniformly locally dominated convergence conditions. This subsection is devoted to a partial improvement of finite atomic characterizations obtained in Section 7.1 by removing the uniformly locally dominated convergence condition as in Definition 7.1 in case $q<\infty$.

By some arguments similar to those used in the proof of the Euclidean case in [7, Lemma 3.6] and (7.1), we immediately obtain the following result; the details are omitted.

Lemma 7.4. Let $\varphi$ be a growth function, $m(\varphi) \leq 0$ and $r \in\left(q(\varphi)[r(\varphi)]^{\prime}, \infty\right]$, where $m(\varphi), q(\varphi)$ and $r(\varphi)$ are, respectively, as in (3.15), (3.16) and (6.9). Then the space

$$
L_{b, 0}^{r}(\mathcal{X}):=\left\{f \in L^{r}(\mathcal{X}): f \text { has bounded support and } \int_{\mathcal{X}} f(x) d \mu(x)=0\right\}
$$

is dense in $H^{\varphi}(\mathcal{X})$.

Now, we extend the Euclidean case in [7, Theorem 3.7] (see also [45, Theorem 4.2] for the anisotropic Euclidean case) to any space of homogeneous type (see Remark 1.1).

Theorem 7.5. Let $\varphi$ be a growth function, $m(\varphi) \leq 0$ and $r \in\left(q(\varphi)[r(\varphi)]^{\prime}, \infty\right]$, where $m(\varphi), q(\varphi)$ and $r(\varphi)$ are, respectively, as in (3.15), (3.16) and (6.9). Then, for any $q \in\left(q(\varphi), \frac{r}{[r(\varphi)]^{\prime}}\right)$ and $f \in L_{b, 0}^{r}(\mathcal{X})$, there exist a finite sequence $\left\{a_{j}\right\}_{j=1}^{N}(N \in \mathbf{N})$ of $(\varphi, q)$-atoms as in Definition 5.2 and numbers $\left\{\lambda_{j}\right\}_{j=1}^{N} \subset \mathbf{C}$ such that

$$
f=\sum_{j=1}^{N} \lambda_{j} a_{j} .
$$

Moreover, there exists a positive constant $C$ such that, for any $f \in L_{b, 0}^{r}(\mathcal{X})$ with the decomposition as above,

$$
\Lambda_{q}\left(\left\{\lambda_{j} a_{j}\right\}_{j=1}^{N}\right) \leq C\|f\|_{H^{\varphi}(\mathcal{X})} .
$$

Proof. Let $q \in\left(q(\varphi), \frac{r}{[r(\varphi)]^{\prime}}\right)$. Then $\left(\frac{r}{q}\right)^{\prime} \in(1, r(\varphi))$ and hence $\varphi \in \mathbf{R H}_{\left(\frac{r}{q}\right)^{\prime}}(\mathcal{X})$. For any $f \in L_{b, 0}^{r}(\mathcal{X})$, there exists a ball $B:=B\left(x_{1}, R\right)$, with $x_{1} \in \mathcal{X}$ and $R \in(0, \infty)$, 
such that supp $f \subset B$. By the Hölder inequality and $\varphi \in \mathbf{R H}_{\left(\frac{r}{q}\right)^{\prime}}(\mathcal{X})$, we know that, for any $t \in(0, \infty)$,

$$
\begin{aligned}
& \frac{1}{\varphi(B, t)} \int_{B}|f(x)|^{q} \varphi(x, t) d \mu(x) \\
& \leq \frac{1}{\varphi(B, t)}\left[\int_{B}[\varphi(x, t)]^{\left(\frac{r}{q}\right)^{\prime}} d \mu(x)\right]^{1 /\left(\frac{r}{q}\right)^{\prime}}\left[\int_{B}|f(x)|^{r} d \mu(x)\right]^{q / r} \\
& \lesssim[\mu(B)]^{-q / r}\left[\int_{B}|f(x)|^{r} d \mu(x)\right]^{q / r}<\infty
\end{aligned}
$$

which, together with Lemma 7.4, further implies that $f \in H^{*, \varphi}(\mathcal{X}) \cap L_{\varphi}^{q}(B)$.

Following the proof of Theorem 7.2(i), with the same notation as therein, we know that

$$
f=\sum_{j \in \mathbf{Z}} \sum_{k \in I_{j}} h_{k}^{j}=\sum_{j \leq \tilde{j}} \sum_{k \in I_{j}} h_{k}^{j}+\sum_{j>\widetilde{j}} \sum_{k \in I_{j}} h_{k}^{j}=: h+\ell
$$

converges both in $\left(\mathcal{G}_{0}^{\eta}(\varrho, \vartheta)\right)^{\prime}$ and almost everywhere on $\mathcal{X}$, where $\widetilde{j}$ is as in $(7.3), h_{k}^{j}$ is a multiple of a $(\varphi, \infty)$-atom as in Definition 5.2 for any $j \in \mathbf{Z}$ and $k \in I_{j}$, $h$ is a multiple of a $(\varphi, \infty)$-atom supported in the ball $\widetilde{B}:=B\left(x_{1}, 16 A_{0} R\right), f^{*} \in L^{1}(\mathcal{X})$ and, for any $x \in \mathcal{X}$,

$$
\sum_{j>\widetilde{j}} \sum_{k \in I_{j}}\left|h_{k}^{j}(x)\right| \lesssim f^{*}(x) \mathbf{1}_{\Omega^{j}}(x) \in L^{1}(\mathcal{X})
$$

For any $N \in \mathbf{N}$, let

$$
F_{N}:=\left\{(k, j): j \in \mathbf{Z}, k \in I_{j}, j>\widetilde{j},|k|+|j| \leq N\right\} \quad \text { and } \quad \ell_{N}:=\sum_{(k, j) \in F_{N}} h_{k}^{j} .
$$

Since $f \in L^{r}(\mathcal{X})$, then, from $\left[27\right.$, Theorem 3.4(ii)], we deduce that $f^{*} \in L^{r}(\mathcal{X})$. By this, (7.8) and the Lebesgue dominated convergence theorem, we conclude that

$$
\lim _{N \rightarrow \infty}\left\|\ell_{N}-\ell\right\|_{L^{r}(\mathcal{X})}=0
$$

which, combined with $(7.7)$ and $\operatorname{supp}(\ell) \cup \operatorname{supp}\left(\ell-\ell_{N}\right) \subset \widetilde{B}$, further implies that

$$
\left\|\ell_{N}-\ell\right\|_{L_{\varphi}^{q}(\widetilde{B})} \leq[\mu(\widetilde{B})]^{-\frac{1}{r}}\left\|\ell_{N}-\ell\right\|_{L^{r}(\widetilde{B})} \rightarrow 0, \quad \text { as } N \rightarrow \infty .
$$

This shows that, for any given $\varepsilon \in(0,1)$, there exists $N \in \mathbf{N}$ such that $\varepsilon^{-1}\left(\ell-\ell_{N}\right)$ is a $(\varphi, q)$-atom supported in the ball $\widetilde{B}$, which implies that $f=h+\ell_{N}+\left(\ell-\ell_{N}\right)$ is a finite linear combination of $(\varphi, \infty)$-atoms and $(\varphi, q)$-atoms and

$$
\Lambda_{q}\left(\left\{h, \ell-\ell_{N}, h_{k}^{j}\right\}_{(k, j) \in F_{N}}\right) \lesssim\|f\|_{H^{*, \varphi}(\mathcal{X})}
$$

This, together with (7.1), then finishes the proof of Theorem 7.5.

As an easy consequence of Theorem 7.5, we obtain the following result, which improves the result in Theorem 7.2(i) by removing the uniformly dominated convergence condition as in Definition 7.1; the details are omitted.

Corollary 7.6. Let $\varphi, r$ and $q$ be as in Theorem 7.5. Then $\|\cdot\|_{H_{\text {fin }}^{\varphi, q}(\mathcal{X})}$ and $\|\cdot\|_{H^{\varphi}(\mathcal{X})}$ are two equivalent quasi-norms on $L_{b, 0}^{r}(\mathcal{X})$. 
As an application, we formulate an improved criterion for the boundedness of quasi-Banach valued sublinear operators on $H^{\varphi}(\mathcal{X})$ in case $q<\infty$, whose proof is similar to that of the Euclidean case in [35, Theorem 3.5]; the details are omitted.

Theorem 7.7. Let $\varphi$ be a growth function of the uniformly upper type $\gamma \in(0,1]$, $m(\varphi) \leq 0$ and $q \in(q(\varphi), \infty)$, where $m(\varphi)$ and $q(\varphi)$ are, respectively, as in (3.15) and (3.16). Suppose that $\mathcal{B}_{\gamma}$ is a $\gamma$-quasi-Banach space. If $T: H_{\text {fin }}^{\varphi, q}(\mathcal{X}) \rightarrow \mathcal{B}_{\gamma}$ is a $\mathcal{B}_{\gamma}$-sublinear operator such that

$$
\sup \left\{\|T a\|_{\mathcal{B}_{\gamma}}: a \text { is a }(\varphi, q) \text {-atom }\right\}<\infty,
$$

then there exists a unique bounded $\mathcal{B}_{\gamma}$-sublinear operator $\widetilde{T}$ from $H^{\varphi}(\mathcal{X})$ into $\mathcal{B}_{\gamma}$ which extends $T$ on $L_{b, 0}^{\infty}(\mathcal{X})$.

Remark 7.8. (i) Theorem 7.7 improves the corresponding result in Theorem 7.3(i) by removing the uniformly locally dominated convergence condition as in Definition 7.1, which has wider applications. Observe that Theorem 7.3(i) is just a simple corollary of Theorem 7.2(i) and, moreover, the proofs of (ii) and (iii) of Theorem 7.2 need to use the proof of Theorem 7.2(i) which is also of independent interest.

(ii) But, in the case $q=\infty$, it is still unclear whether or not the uniformly locally dominated convergence condition in Theorem 7.3(ii) can be removed.

\section{Dual of $\boldsymbol{H}^{\varphi}(\mathcal{X})$}

In this section, we show that the dual space of $H^{\varphi}(\mathcal{X})$ is just the $\operatorname{space} \operatorname{BMO}^{\varphi}(\mathcal{X})$.

Now, we state the following main result of this section, which is a generalization of the corresponding results on Hardy spaces $H^{p}(\mathcal{X})$ in [10, Theorem B] [in this case, $\varphi$ is the same as in (4.2)].

Theorem 8.1. Let $\varphi$ be a growth function as in Definition 2.5 satisfying $m(\varphi) \leq$ 0 and $q \in(q(\varphi), \infty]$, where $m(\varphi)$ and $q(\varphi)$ are respectively as in (3.15) and (3.16). Then the dual space, $\left(H^{\varphi}(\mathcal{X})\right)^{*}$, of $H^{\varphi}(\mathcal{X})$ is $\mathrm{BMO}^{\varphi}(\mathcal{X})$ in the following sense:

(i) Suppose that $b \in \mathrm{BMO}^{\varphi}(\mathcal{X})$. Then the linear functional

$$
L_{b}: f \rightarrow L_{b}(f):=\int_{\mathcal{X}} f(x) b(x) d \mu(x),
$$

initially defined for any $f \in H_{\text {fin }}^{\varphi, q}(\mathcal{X})$, has a bounded linear extension to $H^{\varphi}(\mathcal{X})$.

(ii) Conversely, every continuous linear functional on $H^{\varphi}(\mathcal{X})$ arises as in (i) with a unique $b \in \mathrm{BMO}^{\varphi}(\mathcal{X})$. Moreover, $\|b\|_{\mathrm{BMO}^{\varphi}(\mathcal{X})} \sim\left\|L_{b}\right\|_{\left(H^{\varphi}(\mathcal{X})\right)^{\prime}}$, where the positive equivalence constants are independent of $b$.

Before proving Theorem 8.1, we first state the following lemma which is similar to the Euclidean case in [35, Lemma 4.4] and we omit its proof here.

Lemma 8.2. Let $(\varphi, q)$ be admissible as in Definition 5.2 and $N \in \mathbf{N} \cup\{\infty\}$. Then there exists a positive constant $C$ such that, for any finite sequence $\left\{b_{j}\right\}_{j=1}^{N}$ of multiples of $(\varphi, q)$-atoms,

$$
\sum_{j=1}^{N}\|b\|_{L_{\varphi}^{q}\left(B_{j}\right)}\left\|\mathbf{1}_{B_{j}}\right\|_{L^{\varphi}(\mathcal{X})} \leq C \Lambda\left(\left\{b_{j}\right\}_{j=1}^{N}\right) .
$$


Now, we start to prove Theorem 8.1.

Proof of Theorem 8.1. To prove $\operatorname{BMO}^{\varphi}(\mathcal{X})=\operatorname{Lip}_{\varphi, 1}(\mathcal{X}) \subset\left(H^{\varphi}(\mathcal{X})\right)^{*}$, by Theorem 3.4 and $(7.1)$, it suffices to show that $\operatorname{Lip}_{\varphi, q^{\prime}}(\mathcal{X}) \subset\left(H_{\mathrm{at}}^{\varphi, q}(\mathcal{X})\right)^{*}$, where $q \in(q(\varphi), \infty)$ with $q(\varphi)$ as in (3.16).

Let $g \in \operatorname{Lip}_{\varphi, q^{\prime}}(\mathcal{X})$ and $a$ be a $(\varphi, q)$-atom supported in a ball $B \subset \mathcal{X}$ as in Definition 5.2. Then, by the vanishing moment and the size conditions of $a$, together with the Hölder inequality and $\varphi\left(B,\left\|\mathbf{1}_{B}\right\|_{L^{\varphi}(\mathcal{X})}^{-1}\right)=1$, we conclude that

$$
\begin{aligned}
\mid & \int_{\mathcal{X}} a(x) g(x) d \mu(x) \mid \\
= & \left|\int_{\mathcal{X}} a(x)\left[g(x)-m_{B}(g)\right] d \mu(x)\right| \\
\leq & \int_{\mathcal{X}}|a(x)|\left[\varphi\left(x,\left\|\mathbf{1}_{B}\right\|_{L^{\varphi}(\mathcal{X})}^{-1}\right)\right]^{1 / q}\left[\frac{\left|g(x)-m_{B}(g)\right|}{\varphi\left(x,\left\|\mathbf{1}_{B}\right\|_{L^{\varphi}(\mathcal{X})}^{-1}\right)}\right] \\
& \cdot\left[\varphi\left(x,\left\|\mathbf{1}_{B}\right\|_{L^{\varphi}(\mathcal{X})}^{-1}\right)\right]^{1 / q^{\prime}} d \mu(x) \\
\leq & \|a\|_{L_{\varphi}^{q}(B)} \int_{\mathcal{X}}\left[\frac{\left|g(x)-m_{B}(g)\right|}{\varphi\left(x,\left\|\mathbf{1}_{B}\right\|_{L^{\varphi}(\mathcal{X})}^{-1}\right)}\right]^{q^{\prime}} \varphi\left(x,\left\|\mathbf{1}_{B}\right\|_{L^{\varphi}(\mathcal{X})}^{-1}\right) d \mu(x) \\
\leq & \frac{1}{\left\|\mathbf{1}_{B}\right\|_{L^{\varphi}(\mathcal{X})}} \int_{\mathcal{X}}\left[\frac{\left|g(x)-m_{B}(g)\right|}{\varphi\left(x,\left\|\mathbf{1}_{B}\right\|_{L^{\varphi}(\mathcal{X})}^{-1}\right)}\right]^{q^{\prime}} \varphi\left(x,\left\|\mathbf{1}_{B}\right\|_{L^{\varphi}(\mathcal{X})}^{-1}\right) d \mu(x)=\|g\|_{\operatorname{Lip}_{\varphi, q^{\prime}}(\mathcal{X})} .
\end{aligned}
$$

Now, for any $f \in H_{\text {fin }}^{\varphi, q}(\mathcal{X})=L_{b, 0}^{q}(\mathcal{X})$, by Definition 5.2, we know that there exists a finite sequence $\left\{b_{j}\right\}_{j=1}^{m}$ of multiplies of $(\varphi, q)$-atoms supported, respectively, in balls $\left\{B_{j}\right\}_{j \in \mathbf{N}}$ such that $f:=\sum_{j=1}^{m} b_{j}$ and $\Lambda\left(\left\{b_{j}\right\}_{j=1}^{m}\right) \lesssim\|f\|_{H_{\text {fin }}^{\varphi, q}(\mathcal{X})}$. From this, (8.1), Lemma 8.2 and Theorem 7.2, we deduce that

$$
\begin{aligned}
\left|\int_{\mathcal{X}} f(x) g(x) d \mu(x)\right| & \leq \sum_{j=1}^{m}\|b\|_{L_{\varphi}^{q}\left(B_{j}\right)}\left\|\mathbf{1}_{B_{j}}\right\|_{L^{\varphi}(\mathcal{X})}\|g\|_{\operatorname{Lip}_{\varphi, q^{\prime}}(\mathcal{X})} \lesssim \Lambda_{q}\left(\left\{b_{j}\right\}_{j=1}^{m}\right)\|g\|_{\operatorname{Lip}_{\varphi, q^{\prime}}(\mathcal{X})} \\
& \lesssim\|f\|_{H_{\mathrm{fin}}^{\varphi, q}(\mathcal{X})}\|g\|_{\operatorname{Lip}_{\varphi, q^{\prime}}(\mathcal{X})} \sim\|f\|_{H_{\mathrm{at}}^{\varphi, q}(\mathcal{X})}\|g\|_{\operatorname{Lip}_{\varphi, q^{\prime}}(\mathcal{X})},
\end{aligned}
$$

which, combined with the fact that $L_{b, 0}^{q}(\mathcal{X})$ is dense in $H^{\varphi}(\mathcal{X})$ (see Lemma 7.4), Theorem 7.6 and a standard density argument, further completes the proof of (i).

To prove (ii), let $L \in\left(H^{\varphi}(\mathcal{X})\right)^{*}=\left(H_{\text {at }}^{\varphi, q}(\mathcal{X})\right)^{*}[$ see (7.1)]. For any ball $B \subset \mathcal{X}$ and $q \in(q(\varphi), \infty]$, let

$$
L_{\varphi, 0}^{q}(B):=\left\{f \in L_{\varphi}^{q}(B): \int_{\mathcal{X}} f(x) d \mu(x)=0\right\} .
$$

Obviously, $L_{\varphi, 0}^{q}(B) \subset H^{\varphi}(\mathcal{X})$ and, for any $f \in L_{\varphi, 0}^{q}(B), a:=\left\|\mathbf{1}_{B}\right\|_{L^{\varphi}(\mathcal{X})}^{-1}\|f\|_{L_{\varphi}^{q}(B)}^{-1} f$ is a $(\varphi, q)$-atom supported in $B$ and

$$
\|f\|_{H_{\mathrm{at}}^{\varphi, q}(\mathcal{X})} \leq\left\|\mathbf{1}_{B}\right\|_{L^{\varphi}(\mathcal{X})}\|f\|_{L_{\varphi}^{q}(B)},
$$

which further implies that, for any $L \in\left(H_{\mathrm{at}}^{\varphi, q}(\mathcal{X})\right)^{*}$ and $f \in L_{\varphi, 0}^{q}(B)$,

$$
|L f| \leq\|L\|_{\left(H_{\mathrm{at}}^{\varphi, q}(\mathcal{X})\right)^{*}}\|f\|_{H_{\mathrm{at}}^{\varphi, q}(\mathcal{X})} .
$$

Thus, $L$ is a bounded linear functional on $L_{\varphi, 0}^{q}(B)$. From the Hahn-Banach theorem (see, for instance, [54, p. 77, Corollary 1]), it follows that $L$ can be linearly boundedly extended to the whole space $L_{\varphi}^{q}(B)$ without increasing its norm, which, together 
with the Lebesgue-Radon-Nikodym theorem (see, for instance, [14, Theorem 3.8]), we conclude that there exists $h \in L^{1}(B):=\left\{f \in L^{1}(\mathcal{X})\right.$ : supp $\left.f \subset B\right\}$ such that, for any $f \in L_{\varphi, 0}^{q}(B)$,

$$
L(f)=\int_{\mathcal{X}} f(x) h(x) d \mu(x)
$$

We now choose an increasing sequence $\left\{B_{j}\right\}_{j \in \mathbf{N}}$ of balls such that $B_{1} \subset B_{2} \subset \cdots \subset$ $B_{j} \subset \cdots$ and $\bigcup_{j=1}^{\infty} B_{j}=\mathcal{X}$. From the above arguments, we deduce that there exists a sequence $\left\{h_{j}\right\}_{j \in \mathbf{N}}$ of measurable functions such that, for any $j \in \mathbf{N}, h_{j} \in L^{1}\left(B_{j}\right)$ and, for any $f \in L_{\varphi, 0}^{q}\left(B_{j}\right)$,

$$
L(f)=\int_{\mathcal{X}} f(x) h_{j}(x) d \mu(x) .
$$

Therefore, for any $f \in L_{\varphi, 0}^{q}\left(B_{1}\right)$,

$$
\int_{B_{1}} f(x)\left[h_{1}(x)-h_{2}(x)\right] d \mu(x)=0
$$

which, combined with the fact that $g-m_{B_{1}}(g) \in L_{\varphi, 0}^{q}\left(B_{1}\right)$ for any $g \in L_{\varphi}^{q}\left(B_{1}\right)$, further implies that, for any $g \in L_{\varphi}^{q}\left(B_{1}\right)$,

$$
\int_{B_{1}}\left[g(x)-m_{B_{1}}(g)\right]\left[h_{1}(x)-h_{2}(x)\right] d \mu(x)=0,
$$

here and hereafter, $m_{B_{1}}(g)$ denotes the mean of $g$ on $B_{1}$ as in (2.3) with $f$ and $B$ replaced, respectively, by $g$ and $B_{1}$ here.

Observe that, for any ball $B$ and $f, g \in L_{\varphi}^{q}(B)$,

$$
\int_{B} m_{B}(g)\left[h_{1}(x)-h_{2}(x)\right] d \mu(x)=\int_{B} g(x) m_{B}\left(h_{1}-h_{2}\right) d \mu(x) .
$$

Thus, for any $g \in L_{\varphi}^{q}\left(B_{1}\right)$,

$$
\int_{B_{1}} g(x)\left[h_{1}(x)-h_{2}(x)-m_{B_{1}}\left(h_{1}-h_{2}\right)\right] d \mu(x)=0,
$$

which further shows that, for $\mu$-almost every $x \in B_{1}$,

$$
h_{1}(x)-h_{2}(x)=m_{B_{1}}\left(h_{1}-h_{2}\right) .
$$

Let $\widetilde{h}_{1}:=h_{1}$ and, for any $j \in \mathbf{N}$,

$$
\widetilde{h}_{j+1}:=h_{j+1}+m_{B_{j}}\left(\widetilde{h}_{j}-h_{j+1}\right) .
$$

Then the sequence $\left\{\widetilde{h}_{j}\right\}_{j \in \mathbf{N}}$ satisfies that, for $\mu$-almost every $x \in B_{j}, \widetilde{h}_{j+1}(x)=\widetilde{h}_{j}(x)$ and $\widetilde{h}_{j} \in L^{1}\left(B_{j}\right)$. Let $b$ be a measurable function satisfying that, if $x \in B_{j}$, then $b(x)=\widetilde{h}_{j}(x)$. To finish the proof of Theorem 8.1(ii), it still needs to show that $b \in \operatorname{Lip}_{\varphi, 1}(\mathcal{X})$ and, for any $f \in H_{\text {fin }}^{\varphi, q}(\mathcal{X})$,

$$
L(f)=\int_{\mathcal{X}} f(x) b(x) d \mu(x)
$$

Indeed, for any $f \in H_{\text {fin }}^{\varphi, q}(\mathcal{X})$, we easily know that there exists $j \in \mathbf{N}$ such that supp $f \subset B_{j}$. Thus, $f \in L_{\varphi, 0}^{q}\left(B_{j}\right)$, which, together with (8.2), further implies that

$$
L(f)=\int_{\mathcal{X}} f(x) b(x) d \mu(x)
$$


Furthermore, for any ball $B \subset \mathcal{X}$, let $f:=\operatorname{sign}\left(b-m_{B}(b)\right)$, where $\operatorname{sign}(\cdot)$ denotes the sign function, and

$$
a:=\frac{1}{2}\left\|\mathbf{1}_{B}\right\|_{L^{\varphi}(\mathcal{X})}^{-1}\left[f-m_{B}(f)\right] \mathbf{1}_{B} .
$$

Then $a$ is a $(\varphi, q)$-atom supported in $B$ and

$$
\begin{aligned}
\frac{1}{\left\|\mathbf{1}_{B}\right\|_{L^{\varphi}(\mathcal{X})}} \int_{B}\left|b(x)-m_{B}(b)\right| d \mu(x) & =\frac{1}{\left\|\mathbf{1}_{B}\right\|_{L^{\varphi}(\mathcal{X})}}\left|\int_{B}\left[b(x)-m_{B}(b)\right] f(x) d \mu(x)\right| \\
& =\frac{1}{\left\|\mathbf{1}_{B}\right\|_{L^{\varphi}(\mathcal{X})}}\left|\int_{B}\left[f(x)-m_{B}(f)\right] b(x) d \mu(x)\right| \\
& \lesssim L(a) \lesssim\|L\|_{\left(H_{\mathrm{at}}^{\varphi, q}(\mathcal{X})\right)^{*}}\|a\|_{H_{\mathrm{at}}^{\varphi, q}(\mathcal{X})} \lesssim\|L\|_{\left(H_{\mathrm{at}}^{\varphi, q}(\mathcal{X})\right)^{*}},
\end{aligned}
$$

which further implies that $b \in \operatorname{Lip}_{\varphi, 1}(\mathcal{X})$ and

$$
\|b\|_{\operatorname{Lip}_{\varphi, 1}(\mathcal{X})} \lesssim\|L\|_{\left(H_{\mathrm{at}}^{\varphi, q}(\mathcal{X})\right)^{*}}
$$

This finishes the proof of (ii) and hence of Theorem 8.1.

\section{Boundedness of Calderón-Zygmund operators}

In this section, we establish the boundedness of Calderón-Zygmund operators from $H^{\varphi}(\mathcal{X})$ into $L^{\varphi}(\mathcal{X})$ or from $H^{\varphi}(\mathcal{X})$ to itself, respectively. To this end, we first recall the following notion of Calderón-Zygmund operators from [9] (see also [2, 11]).

Definition 9.1. A function $K \in L_{\text {loc }}^{1}(\{\mathcal{X} \times \mathcal{X}\} \backslash\{(x, x): x \in \mathcal{X}\})$ is called a Calderón-Zygmund kernel if there exists a positive constant $C_{(K)}$, depending on $K$, such that

(i) for any $x, y \in \mathcal{X}$ with $x \neq y$,

$$
|K(x, y)| \leq C_{(K)} \frac{1}{V(x, y)}
$$

(ii) there exists a positive constant $s \in(0,1]$, depending on $K$, such that

(ii) $)_{1}$ for any $x, \widetilde{x}, y \in \mathcal{X}$ with $d(x, y) \geq 2 A_{0} d(x, \widetilde{x})>0$,

$$
|K(x, y)-K(\widetilde{x}, y)| \leq C_{(K)}\left[\frac{d(x, \widetilde{x})}{d(x, y)}\right]^{s} \frac{1}{V(x, y)}
$$

$(\text { ii })_{2}$ for any $x, \widetilde{x}, y \in \mathcal{X}$ with $d(x, y) \geq 2 A_{0} d(y, \widetilde{y})>0$,

$$
|K(x, y)-K(x, \widetilde{y})| \leq C_{(K)}\left[\frac{d(y, \widetilde{y})}{d(x, y)}\right]^{s} \frac{1}{V(x, y)},
$$

where $A_{0}$ is the same as in (1.2).

Let $C(\mathcal{X})$ denote the space of all continuous functions on $\mathcal{X}$ and $s \in(0,1]$. Recall that the space $C_{b}^{s}(\mathcal{X})$ is defined by setting

$$
C_{b}^{s}(\mathcal{X}):=\left\{f \in C^{s}(\mathcal{X}): f \text { has bounded support }\right\},
$$

where the space $C^{s}(\mathcal{X})$ is defined by setting

$$
C^{s}(\mathcal{X}):=\left\{f \in C(\mathcal{X}):\|f\|_{C^{s}(\mathcal{X})}<\infty\right\}
$$

with

$$
\|f\|_{C^{s}(\mathcal{X})}:=\|f\|_{L^{\infty}(\mathcal{X})}+\sup _{\{x, y \in \mathcal{X}: x \neq y\}} \frac{|f(x)-f(y)|}{[d(x, y)]^{s}} .
$$


We equip $C_{b}^{s}(\mathcal{X})$ with the strict inductive limit topology (see, for instance, [50, p. 273] and [26, p. 23]). Moreover, the dual space $\left(C_{b}^{s}(\mathcal{X})\right)^{\prime}$ is defined to be the set of all linear functionals on $C_{b}^{s}(\mathcal{X})$ equipped with the weak-* topology.

Let $T: C_{b}^{s}(\mathcal{X}) \rightarrow\left(C_{b}^{s}(\mathcal{X})\right)^{\prime}$ be a linear continuous operator. Then $T$ is called a Calderón-Zygmund operator with the kernel $K$ satisfying (9.1), (9.2) and (9.3) if, for any $f \in C_{b}^{s}(\mathcal{X})$,

$$
T f(x):=\int_{\mathcal{X}} K(x, y) f(y) d \mu(y), \quad \forall x \in(\operatorname{supp} f)^{\complement} .
$$

Then we state the main result of this section as follows.

Theorem 9.2. Let $\omega$ be as in (1.4) and $s \in(0,1]$ as in Definition 9.1. Suppose that $\varphi$ is a growth function as in Definition 2.5 with $q(\varphi)<\frac{p(s+\omega)}{\omega}$, where $p \in(0,1]$ is as in Definition 2.5(iii) and $q(\varphi)$ as in (3.16), and that $T$ is a Calderón-Zygmund operator as in (9.4) which is bounded on $L^{2}(\mathcal{X})$.

(i) Then there exists a positive constant $C$ such that, for any $f \in H^{\varphi}(\mathcal{X}), T f \in$ $L^{\varphi}(\mathcal{X})$ and $\|T f\|_{L^{\varphi}(\mathcal{X})} \leq C\|f\|_{H^{\varphi}(\mathcal{X})}$.

(ii) If we further assume that $T^{*} 1=0$, then there exists a positive constant $\widetilde{C}$ such that, for any $f \in H^{\varphi}(\mathcal{X}), T f \in H^{\varphi}(\mathcal{X})$ and $\|T f\|_{H_{\mathrm{at}}^{\varphi}(\mathcal{X})} \leq \widetilde{C}\|f\|_{H^{\varphi}(\mathcal{X})}$.

Proof. Choose $r \in[2, \infty)$ such that $r^{\prime}<r(\varphi)$. Let $(\varphi, q)$ be admissible as in Definition 5.2 and $a$ a $(\varphi, q)$-atom with $q \in(r q(\varphi), \infty)$, supported in the ball $B_{0}:=B\left(x_{0}, r_{0}\right)$ for some $x_{0} \in \mathcal{X}$ and $r_{0} \in(0, \infty)$ as in Definition 5.2.

We first prove (i). By Theorem 7.7, it suffices to show that $\|T a\|_{L^{\varphi}(\mathcal{X})} \lesssim 1$. Indeed, we write

$$
\|T a\|_{L^{\varphi}(\mathcal{X})} \lesssim\left\|\mathbf{1}_{2 A_{0} B_{0}} T a\right\|_{L^{\varphi}(\mathcal{X})}+\left\|\mathbf{1}_{\left(2 A_{0} B_{0}\right)^{\mathrm{c}}} T a\right\|_{L^{\varphi}(\mathcal{X})}=: \mathrm{I}+\mathrm{II} .
$$

For I, by the boundedness of $T$ on $L^{r}(\mathcal{X})$, we know that

$$
\|T a\|_{L^{r}(\mathcal{X})} \lesssim\|a\|_{L^{r}(\mathcal{X})} .
$$

Meanwhile, from $\frac{q}{r} \in(q(\varphi), \infty)$, it follows that $\varphi \in \mathbf{A}_{\frac{q}{r}}(\mathcal{X})$, which, combined with Lemma 2.6(iii), implies that

$$
\begin{aligned}
\left\{\frac{1}{\mu\left(B_{0}\right)} \int_{B_{0}}|a(x)|^{r} d \mu(x)\right\}^{1 / r} & \lesssim\left\{\frac{1}{\varphi\left(B_{0}, t\right)} \int_{B_{0}}|a(x)|^{q} \varphi(x, t) d \mu(x)\right\}^{1 / q} \\
& \lesssim\|a\|_{L_{\varphi}^{q}(\mathcal{X})} \lesssim \frac{1}{\left\|\mathbf{1}_{B_{0}}\right\|_{L^{\varphi}(\mathcal{X})}}
\end{aligned}
$$

By this and (9.5), we conclude that

$$
\|T a\|_{L^{r}(\mathcal{X})} \lesssim\|a\|_{L^{r}(\mathcal{X})} \lesssim \frac{\left[\mu\left(B_{0}\right)\right]^{1 / r}}{\left\|\mathbf{1}_{B_{0}}\right\|_{L^{\varphi}(\mathcal{X})}}
$$

which, together with the arguments used in the estimation of $\mathrm{A}$ in the proof of Theorem 6.15, we conclude that

$$
\int_{2 A_{0} B} \varphi(x, T(\lambda a)(x)) d \mu(x) \lesssim \varphi\left(B, \frac{|\lambda|}{\left\|\mathbf{1}_{B}\right\|_{L^{\varphi}(\mathcal{X})}}\right) .
$$

This further shows that $\mathrm{I} \lesssim 1$. 
To estimate II, from $\int_{\mathcal{X}} a(y) d \mu(y)=0,(9.3)$, the Hölder inequality and (9.6), we deduce that, for any $x \in\left(2 A_{0} B_{0}\right)^{\complement}$,

$$
\begin{aligned}
|T a(x)| & =\left|\int_{\mathcal{X}}\left[K(x, y)-K\left(x, x_{0}\right)\right] a(y) d \mu(y)\right| \\
& \leq \int_{\mathcal{X}}\left|K(x, y)-K\left(x, x_{0}\right)\right||a(y)| d \mu(y) \\
& \lesssim \int_{B_{0}}\left[\frac{d\left(y, x_{0}\right)}{d\left(x, x_{0}\right)}\right]^{s} \frac{1}{V\left(x, x_{0}\right)}|a(y)| d \mu(y) \\
& \lesssim\left[\frac{r_{0}}{d\left(x, x_{0}\right)}\right]^{s} \frac{1}{V\left(x, x_{0}\right)}\|a\|_{L^{1}(\mathcal{X})} \\
& \lesssim\left[\frac{r_{0}}{d\left(x, x_{0}\right)}\right]^{s} \frac{1}{V\left(x, x_{0}\right)}\|a\|_{L^{r}(\mathcal{X})}\left[\mu\left(B_{0}\right)\right]^{\frac{1}{r^{\prime}}} \\
& \lesssim\left[\frac{r_{0}}{d\left(x, x_{0}\right)}\right]^{s} \frac{1}{V\left(x, x_{0}\right)} \frac{\mu\left(B_{0}\right)}{\left\|\mathbf{1}_{B_{0}}\right\|_{L^{\varphi}(\mathcal{X})}} \\
& \lesssim\left[\frac{\mu\left(B_{0}\right)}{V\left(x, x_{0}\right)}\right]^{\frac{s}{\omega}+1} \frac{1}{\left\|\mathbf{1}_{B_{0}}\right\|_{L^{\varphi}(\mathcal{X})}} \lesssim \frac{\left[M\left(\mathbf{1}_{B_{0}}\right)(x)\right]^{\frac{s}{\omega}+1}}{\left\|\mathbf{1}_{B_{0}}\right\|_{L^{\varphi}(\mathcal{X})}}
\end{aligned}
$$

which, combined with Theorem 4.11 and $q(\varphi)<\frac{p(s+\omega)}{\omega}$, further implies that

$$
\begin{aligned}
\mathrm{II} & \lesssim\left\|\left[\left(\frac{\mathbf{1}_{B_{0}}}{\left\|\mathbf{1}_{B_{0}}\right\|_{L^{\varphi}(\mathcal{X})}^{\frac{\omega}{\omega+s}}}\right)\right]^{\frac{s}{\omega}+1}\right\|_{L^{\varphi}(\mathcal{X})} \sim\left\|M\left(\frac{\mathbf{1}_{B_{0}}}{\left\|\mathbf{1}_{B_{0}}\right\|_{L^{\varphi}(\mathcal{X})}^{\frac{\omega}{\omega+s}}}\right)\right\|_{L^{\widetilde{\varphi}(\mathcal{X})}}^{\frac{\omega+s}{\omega}} \\
& \lesssim\left\|\frac{\mathbf{1}_{B_{0}}}{\left\|\mathbf{1}_{B_{0}}\right\|_{L^{\varphi}(\mathcal{X})}^{\frac{\omega}{\omega+s}}}\right\|_{L^{\tilde{\varphi}(\mathcal{X})}}^{\frac{\omega+s}{\omega}} \sim\left\|\frac{\mathbf{1}_{B_{0}}}{\left\|\mathbf{1}_{B_{0}}\right\|_{L^{\varphi}(\mathcal{X})}}\right\|_{L^{\varphi}(\mathcal{X})} \sim 1,
\end{aligned}
$$

where, for any $x \in \mathcal{X}$ and $t \in(0, \infty), \widetilde{\varphi}(x, t):=\varphi\left(x, t^{\frac{s+\omega}{\omega}}\right)$ is of uniformly lower type $\frac{p(s+\omega)}{\omega}$.

Combining the estimates of I and II, we have

$$
\|T a\|_{L^{\varphi}(\mathcal{X})} \lesssim \mathrm{I}+\mathrm{II} \lesssim 1
$$

which completes the proof of (i).

Now, we show (ii). Similarly to (i), by Theorem 7.7, we only need to prove that $\|T a\|_{H_{\mathrm{at}}^{\varphi}(\mathcal{X})} \lesssim 1$. Indeed, we show that $T a$ is a $(\varphi, r, s)$-molecule related to the ball $2 A_{0} B_{0}$ as in Definition 6.7. To this end, we write

$$
T a=\sum_{j=0}^{\infty} \mathbf{1}_{U_{j}\left(2 A_{0} B_{0}\right)} T a,
$$

where $U_{0}\left(2 A_{0} B_{0}\right):=2 A_{0} B_{0}$ and, for any $j \in \mathbf{N}, U_{j}\left(2 A_{0} B_{0}\right):=\delta^{-j} 2 A_{0} B_{0} \backslash \delta^{-j+1} 2 A_{0} B_{0}$. From (9.6) and some arguments similar to those used in the proof of [43, Theorem 2.7], it follows that

$$
\|T a\|_{L^{r}(\mathcal{X})} \lesssim \frac{\left[\mu\left(B_{0}\right)\right]^{1 / r}}{\left\|\mathbf{1}_{B_{0}}\right\|_{L^{\varphi}(\mathcal{X})}} \lesssim \frac{\left[\mu\left(2 A_{0} B_{0}\right)\right]^{1 / r}}{\left\|\mathbf{1}_{2 A_{0} B_{0}}\right\|_{L^{\varphi}(\mathcal{X})}}
$$


Moreover, by the proof of (9.7) and some arguments similar to those used in the proof of [43, Theorem 2.7], we know that, for any $x \in\left(2 A_{0} B_{0}\right)^{\complement}$,

$$
|T a(x)| \lesssim\left[\frac{r_{0}}{d\left(x, x_{0}\right)}\right]^{s} \frac{1}{V\left(x, x_{0}\right)} \frac{\mu\left(B_{0}\right)}{\left\|\mathbf{1}_{B_{0}}\right\|_{L^{\varphi}(\mathcal{X})}} \lesssim\left[\frac{r_{0}}{d\left(x, x_{0}\right)}\right]^{s} \frac{1}{V\left(x, x_{0}\right)} \frac{\mu\left(2 A_{0} B_{0}\right)}{\left\|\mathbf{1}_{2 A_{0} B_{0}}\right\|_{L^{\varphi}(\mathcal{X})}},
$$

which further implies that, for any $j \in \mathbf{N}$,

$$
\begin{aligned}
& \|T a\|_{L^{r}\left(U_{j}\left(2 A_{0} B_{0}\right)\right)} \\
& \lesssim\left\{\int_{\delta^{-j} 2 A_{0} B_{0} \backslash \delta^{-j+1} 2 A_{0} B_{0}}\left[\frac{r_{0}}{d\left(x, x_{0}\right)}\right]^{r s} \frac{1}{\left[V\left(x, x_{0}\right)\right]^{r}} d \mu(x)\right\}^{\frac{1}{r}} \frac{\mu\left(2 A_{0} B_{0}\right)}{\left\|\mathbf{1}_{2 A_{0} B_{0}}\right\|_{L^{\varphi}(\mathcal{X})}} \\
& \lesssim \delta^{j s} \frac{\left[\mu\left(\delta^{-j} 2 A_{0} B_{0}\right)\right]^{1 / r}}{V\left(x_{0}, \delta^{-j+1} 2 A_{0} r_{0}\right)} \frac{\mu\left(2 A_{0} B_{0}\right)}{\left\|\mathbf{1}_{2 A_{0} B_{0}}\right\|_{L^{\varphi}(\mathcal{X})}} \lesssim \delta^{j s} \frac{\left[\mu\left(\delta^{-j} 2 A_{0} B_{0}\right)\right]^{1 / r}}{\left\|\mathbf{1}_{2 A_{0} B_{0}}\right\|_{L^{\varphi}(\mathcal{X})}} .
\end{aligned}
$$

On another hand, by $T^{*} 1=0$, we immediately obtain $\int_{\mathcal{X}} T a(x) d \mu(x)=0$. Thus, $T a$ is a harmless positive constant multiple of a $(\varphi, r, s)$-molecule related to the ball $2 A_{0} B_{0}$, which, together with Theorem 6.8 , further implies that

$$
\|T a\|_{H_{\mathrm{at}}^{\varphi}(\mathcal{X})} \sim\|T a\|_{H_{\mathrm{mol}}^{\varphi, r, s}(\mathcal{X})} \lesssim 1
$$

This finishes the proof of (ii) and hence of Theorem 9.2.

Remark 9.3. Let all the notation be the same as in Theorem 9.2. We point out that the condition $q(\varphi)<\frac{p(s+\omega)}{\omega}$ in Theorem 9.2 is sharp in the sense that, if $\varphi(x, t):=t^{p}$ for any $(x, t) \in \mathcal{X} \times[0, \infty)$, then $q(\varphi)=1$ and hence $q(\varphi)<\frac{p(s+\omega)}{\omega}$ if and only if $p>\frac{\omega}{s+\omega}$, which returns to the classical case (see, for instance, [47, Theorem 3.4]).

\section{Class of pointwise multipliers for $\operatorname{BMO}(\mathcal{X})$}

In this section, we establish a new characterization of $\operatorname{PWM}(\operatorname{BMO}(\mathcal{X}))$, namely, the set of all pointwise multipliers of the space $\operatorname{BMO}(\mathcal{X})$.

It was known by $[31$, p. 1925] that $\varphi$ in $(2.4)$ satisfies $\omega q(\varphi)<(\omega+1) i(\varphi)$. More precisely, $\varphi \in \mathbf{A}_{1}(\mathcal{X})$ and, for almost every fixed $x \in \mathcal{X}, \varphi(x, \cdot)$ is concave with $i(\varphi)=1$.

The following definition of log-atoms on $\mathcal{X}$ is a variant of log-atoms in the Euclidean case from [35, Section 7].

Definition 10.1. A measurable function $a$ is called a log-atom if it satisfies the following three conditions:

(i) supp $a \subset B$ for some ball $B$ in $\mathcal{X}$;

(ii) $\|a\|_{L^{\infty}(\mathcal{X})} \leq \frac{1}{\mu(B)}\left[\log \left(e+\frac{1}{\mu(B)}\right)+\sup _{x \in B} \log \left(e+d\left(x_{0}, x\right)\right)\right]$ with $x_{0}$ as in Remark 2.10;

(iii) $\int_{\mathcal{X}} a(x) d \mu(x)=0$.

We first show the following technical proposition, which is a generalization of the Euclidean case in [35, Proposition 7.1] to any space of homogeneous type. In what follows, we always let $\varphi$ be as in (2.4).

Proposition 10.2. There exists a positive constant $\widetilde{C}$ such that, if $f$ is a $(\varphi, \infty)$ atom as in Definition 5.2 [resp., log-atom as in Definition 10.1], then $\widetilde{C}^{-1} f$ is a log-atom [resp., $(\varphi, \infty)$-atom]. 
Proof. Let $f$ be a $(\varphi, \infty)$-atom supported in a ball $B \subset \mathcal{X}$. We first show that $\frac{1}{4} f$ is a log-atom. Indeed, to this end, it suffices to show that $\frac{1}{4} f$ satisfies Definition 10.1(ii). Observe that

$$
\begin{gathered}
4\left[\log \left(e+\frac{1}{\mu(B)}\right)+\sup _{x \in B} \log \left(e+d\left(x_{0}, x\right)\right)\right]\left[\operatorname { l o g } \left(e+\frac{4}{\mu(B)}\left\{\log \left(e+\frac{1}{\mu(B)}\right)\right.\right.\right. \\
\left.\left.\left.+\sup _{x \in B} \log \left(e+d\left(x_{0}, x\right)\right)\right\}\right)+\sup _{x \in B} \log \left(e+d\left(x_{0}, x\right)\right)\right]^{-1} \geq 1 .
\end{gathered}
$$

By this, we conclude that

$$
\int_{B} \varphi\left(x, \frac{4}{\mu(B)}\left[\log \left(e+\frac{1}{\mu(B)}\right)+\sup _{x \in B} \log \left(e+d\left(x_{0}, x\right)\right)\right]\right) d \mu(x) \geq 1,
$$

which implies that

$$
\begin{aligned}
\left\|\frac{1}{4} f\right\|_{L^{\infty}(\mathcal{X})} & \leq \frac{1}{4}\left\|\mathbf{1}_{B}\right\|_{L^{\varphi}(\mathcal{X})}^{-1} \leq \frac{1}{4} \frac{4}{\mu(B)}\left[\log \left(e+\frac{1}{\mu(B)}\right)+\sup _{x \in B} \log \left(e+d\left(x_{0}, x\right)\right)\right] \\
& =\frac{1}{\mu(B)}\left[\log \left(e+\frac{1}{\mu(B)}\right)+\sup _{x \in B} \log \left(e+d\left(x_{0}, x\right)\right)\right] .
\end{aligned}
$$

This is the desired conclusion.

On another hand, let $f$ be a log-atom. Then we prove that $f$ is a multiple of a $(\varphi, \infty)$-atom. We also observe that

$$
\begin{aligned}
& {\left[\log \left(e+\frac{1}{\mu(B)}\right)+\sup _{x \in B} \log \left(e+d\left(x_{0}, x\right)\right)\right]\left[\operatorname { l o g } \left(e+\frac{1}{\mu(B)}\left\{\log \left(e+\frac{1}{\mu(B)}\right)\right.\right.\right.} \\
& \left.\left.\left.\quad+\sup _{x \in B} \log \left(e+d\left(x_{0}, x\right)\right)\right\}\right)+\sup _{x \in B} \log \left(e+d\left(x_{0}, x\right)\right)\right]^{-1} \leq 1 .
\end{aligned}
$$

By this and $\varphi \in \mathbf{A}_{1}(\mathcal{X})$, we find that

$$
\int_{B} \varphi\left(x, \frac{1}{\mu(B)}\left[\log \left(e+\frac{1}{\mu(B)}\right)+\sup _{x \in B} \log \left(e+d\left(x_{0}, x\right)\right)\right]\right) d \mu(x) \lesssim 1
$$

which implies that

$$
\|f\|_{L^{\infty}(\mathcal{X})} \leq \frac{1}{\mu(B)}\left[\log \left(e+\frac{1}{\mu(B)}\right)+\sup _{x \in B} \log \left(e+d\left(x_{0}, x\right)\right)\right] \lesssim\left\|\mathbf{1}_{B}\right\|_{L^{\varphi}(\mathcal{X})}^{-1} .
$$

This finishes the proof of Proposition 10.2.

The following definition of weighted BMO spaces is taken from [52].

Definition 10.3. Let $\mathrm{BMO}_{\phi}(\mathcal{X})$ be the space of all $f \in L_{\text {loc }}^{1}(\mathcal{X})$ such that, for any $a \in \mathcal{X}$ and $r \in(0, \infty)$,

$$
\mathrm{MO}_{\phi}(f, B(a, r)):=\frac{1}{\phi(a, r)} \frac{1}{V(a, r)} \int_{B(a, r)}\left|f(x)-m_{B(a, r)}(f)\right| d \mu(x)<\infty,
$$

where $\phi(a, r):=\frac{1}{\log \left(d\left(x_{0}, a\right)+r+1 / r\right)}$ and

$$
m_{B(a, r)}(f):=\frac{1}{\mu(B(a, r))} \int_{B(a, r)} f(y) d \mu(y) .
$$

For any $f \in L_{\text {loc }}^{1}(\mathcal{X})$, let $\|f\|_{\mathrm{BMO}_{\phi}(\mathcal{X})}:=\sup _{\{a \in \mathcal{X}, r \in(0, \infty)\}} \mathrm{MO}_{\phi}(f, B(a, r))$ and

$$
\|f\|_{\mathrm{bmo}_{\phi}(\mathcal{X})}:=\|f\|_{\mathrm{BMO}_{\phi}(\mathcal{X})}+\left|m_{B\left(x_{0}, 1\right)}(f)\right| \text {, }
$$

where $x_{0}$ is as in (2.4). 
In what follows, we need the following Ahlfors n-regular condition on the measure $\mu$, namely, there exists $n \in(0, \infty)$ such that, for any ball $B(x, r)$ with $x \in \mathcal{X}$ and $r \in(0, \infty)$,

$$
V(x, r) \sim r^{n}
$$

where the positive equivalence constants are independent of $x$ and $r$.

The following result is a generalization of the corresponding Euclidean case in [35, Proposition 7.2].

Proposition 10.4. Assume that $\mu$ satisfies the Ahlfors $n$-regular condition (10.1). For any $f \in \mathrm{BMO}_{\phi}(\mathcal{X})$, it holds true that

$\|f\|_{\mathrm{BMO}_{\phi}(\mathcal{X})}$

$\sim \sup _{B: \text { ball }} \frac{1}{\mu(B)}\left[\log \left(e+\frac{1}{\mu(B)}\right)+\sup _{x \in B} \log \left(e+d\left(x_{0}, x\right)\right)\right] \int_{B}\left|f(x)-m_{B}(f)\right| d \mu(x)<\infty$ with the positive equivalence constants independent of $f$.

Proof. It suffices to show that, for any ball $B:=B(a, r)$ with $a \in \mathcal{X}$ and $r \in(0, \infty)$,

$$
\log \left(d\left(x_{0}, a\right)+r+\frac{1}{r}\right) \sim \log \left(e+\frac{1}{\mu(B)}\right)+\sup _{x \in B} \log \left(e+d\left(x_{0}, x\right)\right) .
$$

We first show that

$$
\log \left(e+\frac{1}{\mu(B)}\right)+\sup _{x \in B} \log \left(e+d\left(x_{0}, x\right)\right) \lesssim \log \left(d\left(x_{0}, a\right)+r+\frac{1}{r}\right) .
$$

Indeed, by (10.1), we have

$$
\log \left(e+\frac{1}{\mu(B)}\right) \sim \log \left(e+\frac{1}{r^{n}}\right) \lesssim \log \left(e+\frac{1}{r}\right) \lesssim \log \left(d\left(x_{0}, a\right)+r+\frac{1}{r}\right)
$$

and we also observe that

$$
\sup _{x \in B} \log \left(e+d\left(x_{0}, x\right)\right) \leq \log \left(e+A_{0} d\left(x_{0}, a\right)+A_{0} r\right) \lesssim \log \left(d\left(x_{0}, a\right)+r+\frac{1}{r}\right),
$$

which implies the desired conclusion.

Conversely, we show that

$$
\log \left(d\left(x_{0}, a\right)+r+\frac{1}{r}\right) \lesssim \log \left(e+\frac{1}{\mu(B)}\right)+\sup _{x \in B} \log \left(e+d\left(x_{0}, x\right)\right) .
$$

It is easy to see that $(\mathcal{X}, d, \mu)$ with $\mu$ satisfying (10.1) is an RD-space, which further implies that there exists a constant $a_{0} \in(1, \infty)$ such that, for any $a \in \mathcal{X}$ and $r \in(0, \infty), B(a, r) \backslash B\left(a, \frac{r}{a_{0}}\right) \neq \emptyset$. Thus, we are able to choose $y_{0} \in B(a, r) \backslash B\left(a, \frac{r}{a_{0}}\right)$. Therefore, we have

$$
\frac{r}{a_{0}} \leq d\left(y_{0}, a\right)<r
$$

We further consider the following two cases.

Case (i) $r \in(0,1]$. In this case, by (10.1), we obtain

$$
\begin{aligned}
\log \left(d\left(x_{0}, a\right)+r+\frac{1}{r}\right) & \leq \log \left(d\left(x_{0}, a\right)+\frac{2}{r}\right) \lesssim \log \left(e+\frac{1}{r}\right)+\log \left(e+d\left(x_{0}, a\right)\right) \\
& \lesssim \log \left(e+\frac{1}{\mu(B)}\right)+\sup _{x \in B} \log \left(e+d\left(x_{0}, x\right)\right) .
\end{aligned}
$$


Case (ii) $r \in(1, \infty)$. In this case, by $(10.2)$, we have

$$
\begin{aligned}
\log \left(d\left(x_{0}, a\right)+r+\frac{1}{r}\right) & \leq \log \left(d\left(x_{0}, a\right)+2 r\right) \lesssim \log \left(e+d\left(x_{0}, a\right)+d\left(y_{0}, a\right)\right) \\
& \lesssim \sup _{x \in B} \log \left(e+d\left(x_{0}, x\right)\right),
\end{aligned}
$$

which then completes the proof of Proposition 10.4.

The following corollary gives an equivalent characterization of the $\operatorname{BMO}^{\log }(\mathcal{X})$ norm.

Corollary 10.5. Let $\mu$ satisfy the Ahlfors n-regular condition (10.1). Then both $\mathrm{BMO}^{\log }(\mathcal{X})$ and $\mathrm{BMO}_{\phi}(\mathcal{X})$ coincide with equivalent norms. ball $B$,

Proof. Let $\varphi$ be as in (2.4). It was shown by [31, Remark 4.1(iii)] that, for any

$$
\left\|\mathbf{1}_{B}\right\|_{L^{\varphi}(\mathcal{X})} \sim \frac{\mu(B)}{\log \left(e+\frac{1}{\mu(B)}\right)+\sup _{x \in B} \log \left(e+d\left(x_{0}, x\right)\right)},
$$

which, combined with Proposition 10.4, immediately completes the proof of Corollary 10.5 .

Now, we state the main result of this section as follows, which is a generalization of the corresponding Euclidean case in [35, Theorem 3.3].

Theorem 10.6. Assume that $\mu$ satisfies the Ahlfors n-regular condition (10.1). The set of pointwise multipliers for $\operatorname{BMO}(\mathcal{X})$, denoted by $\operatorname{PWM}(\operatorname{BMO}(\mathcal{X}))$, is the dual space of $L^{1}(\mathcal{X})+H^{*, \varphi}(\mathcal{X})$, where $\varphi$ is as in (2.4).

Proof. From [52, Example 2.8], it follows that $\operatorname{PWM}(\operatorname{BMO}(\mathcal{X}))=\left[L^{\infty}(\mathcal{X}) \cap\right.$ $\left.\mathrm{BMO}^{\varphi}(\mathcal{X})\right]$. Thus, we only need to prove that $\left[L^{\infty}(\mathcal{X}) \cap \mathrm{BMO}^{\varphi}(\mathcal{X})\right]=\left(L^{1}(\mathcal{X})+\right.$ $\left.H^{*, \varphi}(\mathcal{X})\right)^{*}$.

From Propositions 10.2 and 10.4, Corollary 10.5 and some arguments similar to those used in the proof of [35, Theorem 3.3], we deduce that the above conclusion obviously holds true, which completes the proof of Theorem 10.6.

Acknowledgements. The authors would like to thank the referee for her/his careful reading of the manuscript and giving many constructive comments which indeed improve the presentation of this article and they would also like to thank Ziyi He for some helpful discussions on the proof of (6.20).

\section{References}

[1] Andersen, K. F., and R. T. John: Weighted inequalities for vector-valued maximal functions and singular integrals. - Studia Math. 69, 1980/81, 19-31.

[2] Auscher, P., and T. Hytönen: Orthonormal bases of regular wavelets in spaces of homogeneous type. - Appl. Comput. Harmon. Anal. 34, 2013, 266-296.

[3] Auscher, P., and T. Hytönen: Addendum to Orthonormal bases of regular wavelets in spaces of homogeneous type [Appl. Comput. Harmon. Anal. 34:2, 2013, 266-296]. - Appl. Comput. Harmon. Anal. 39, 2015, 568-569.

[4] Bonami, A., J. Feuto, and S. Grellier: Endpoint for the DiV-CURL lemma in Hardy spaces. - Publ. Mat. 54, 2010, 341-358.

[5] Bonami, A., S. Grellier, and L. D. KY: Paraproducts and products of functions in $\operatorname{BMO}\left(\mathbf{R}^{n}\right)$ and $H^{1}\left(\mathbf{R}^{n}\right)$ through wavelets. - J. Math. Pures Appl. (9) 97, 2012, 230-241. 
[6] Bonami, A., T. Iwaniec, P. Jones, and M. Zinsmeister: On the product of functions in BMO and $H^{1}$. - Ann. Inst. Fourier (Grenoble) 57, 2007, 1405-1439.

[7] Bonami, A., L. D. Ky, Y. Liang, and D. Yang: Several remarks on Musielak-Orlicz Hardy spaces. - Manuscript.

[8] Coifman, R. R.: A real variable characterization of $H^{p}$. - Studia Math. 51, 1974, 269-274.

[9] Colfman, R. R., and G. Weiss: Analyse harmonique non-commutative sur certains espaces homogènes. Étude de certaines intégrales singulières. - Lecture Notes in Math. 242, SpringerVerlag, Berlin-New York, 1971 (in French).

[10] Coifman, R. R., and G. Weiss: Extensions of Hardy spaces and their use in analysis. - Bull. Amer. Math. Soc. 83, 1977, 569-645.

[11] Deng, D., and Y. HAN: Harmonic analysis on spaces of homogeneous type. - Lecture Notes in Math. 1966, Springer-Verlag, Berlin, 2009.

[12] Diening, L., P. Harjulehto, P. HÄstö, and M. RůžıčKa: Lebesgue and Sobolev spaces with variable exponents. - Lecture Notes in Math. 2017, Springer, Heidelberg, 2011.

[13] Fefferman, C., and E. M. Stein: $H^{p}$ spaces of several variables. - Acta Math. 129, 1972, $137-193$.

[14] Folland, G. B.: Real analysis. Modern techniques and their applications. Second edition. Pure Appl. Math. (New York), A Wiley-Interscience Publication, John Wiley \& Sons, Inc., New York, 1999.

[15] Fu, X., D.-C. Chang, and D. YAng: Recent progress in bilinear decompositions. - Applied Analysis and Optimization 1, 2017, 153-210.

[16] Fu, X., and D. YAng: Products of functions in $H_{\rho}^{1}(\mathcal{X})$ and $\mathrm{BMO}_{\rho}(X)$ over RD-spaces and applications to Schrödinger operators. - J. Geom. Anal. 27, 2017, 2938-2976.

[17] Fu, X., and D. YAng: Wavelet characterizations of the atomic Hardy space $H^{1}$ on spaces of homogeneous type. - Appl. Comput. Harmon. Anal. 44, 2018, 1-37.

[18] Fu, X., and D. YANG: Wavelet characterizations of Musielak-Orlicz Hardy spaces. - Banach J. Math. Anal. 12, 2018, 1017-1046.

[19] Fu, X., D. YAng, and Y. Liang: Products of functions in $\operatorname{BMO}(\mathcal{X})$ and $H_{\text {at }}^{1}(\mathcal{X})$ via wavelets over spaces of homogeneous type. - J. Fourier Anal. Appl. 23, 2017, 919-990.

[20] García-Cuerva, J., and J. M. MArtell: Wavelet characterization of weighted spaces. - J. Geom. Anal. 11, 2001, 241-264.

[21] Grafakos, L., L. Liu, and D. Yang: Maximal function characterizations of Hardy spaces on RD-spaces and their applications. - Sci. China Ser. A 51, 2008, 2253-2284.

[22] Grafakos, L., L. LiU, and D. YANG: Vector-valued singular integrals and maximal functions on spaces of homogeneous type. - Math. Scand. 104, 2009, 296-310.

[23] Han, Ya., Han, Yo., and J. Li: Criterion of the boundedness of singular integrals on spaces of homogeneous type. - J. Funct. Anal. 271, 2016, 3423-3464.

[24] HAn, Y., J. Li, and L. A. WARD: Hardy space theory on spaces of homogeneous type via orthonormal wavelet bases. - Appl. Comput. Harmon. Anal. 45, 2018, 120-169.

[25] HAN, Y., D. MÜller, and D. YANG: Littlewood-Paley characterizations for Hardy spaces on spaces of homogeneous type. - Math. Nachr. 279, 2006, 1505-1537.

[26] Han, Y., D. Müller, and D. YANG: A theory of Besov and Triebel-Lizorkin spaces on metric measure spaces modeled on Carnot-Carathéodory spaces. - Abstr. Appl. Anal. 2008, Art. ID 893409, $250 \mathrm{pp}$.

[27] He, Z., Y. Han, J. Li, L. LiU, D. YANG, and W. YuAN: A complete real-variable theory of Hardy spaces on spaces of homogeneous type. - J. Fourier Anal. Appl. 25, 2019, 2197-2267.

[28] He, Z., L. Liu, D. YAng, and W. YuAn: New Calderón reproducing formulae with exponential decay on spaces of homogeneous type. - Sci. China Math. 62, 2019, 283-350. 
[29] Heinonen, J.: Lectures on analysis on metric spaces. - Springer-Verlag, New York, 2001.

[30] Hou, S., D. YANG, and S. YANG: Lusin area function and molecular characterizations of Musielak-Orlicz Hardy spaces and their applications. - Commun. Contemp. Math. 15, 2013, 1350029, 37 pp.

[31] Hou, S., D. YANG, and S. YANG: Musielak-Orlicz BMO-type spaces associated with generalized approximations to the identity. - Acta Math. Sin. (Engl. Ser.) 30, 2014, 1917-1962.

[32] Hytönen, T., and A. Kairema: Systems of dyadic cubes in a doubling metric space. - Colloq. Math. 126, 2012, 1-33.

[33] John, F., and L. Nirenberg: On functions of bounded mean oscillation. - Comm. Pure Appl. Math. 14, 1961, 415-426.

[34] KY, L. D.: Bilinear decompositions and commutators of singular integral operators. - Trans. Amer. Math. Soc. 365, 2013, 2931-2958.

[35] Ky, L. D.: New Hardy spaces of Musielak-Orlicz type and boundedness of sublinear operators. - Integral Equations Operator Theory 78, 2014, 115-150.

[36] KY, L. D.: Bilinear decompositions for the product space $H_{L}^{1} \times \mathrm{BMO}_{L}$. - Math. Nachr. 287, 2014, 1288-1297.

[37] Ky, L. D.: Endpoint estimates for commutators of singular integrals related to Schrödinger operators. - Rev. Mat. Iberoam. 31, 2015, 1333-1373.

[38] KY, L. D.: On the product of functions in BMO and $H^{1}$ over spaces of homogeneous type. J. Math. Anal. Appl. 425, 2015, 807-817.

[39] Latter, R. H.: A characterization of $H^{p}\left(\mathbf{R}^{n}\right)$ in terms of atoms. - Studia Math. 62, 1978, 93-101.

[40] LI, W.: A maximal function characterization of Hardy spaces on spaces of homogeneous type. - Approx. Theory Appl. (N.S.) 14, 1998, 12-27.

[41] Liang, Y., J. HuAng, and D. Yang: New real-variable characterizations of Musielak-Orlicz Hardy spaces. - J. Math. Anal. Appl. 395, 2012, 413-428.

[42] Liang, Y., E. Nakai, D. Yang, and J. Zhang: Boundedness of intrinsic Littlewood-Paley functions on Musielak-Orlicz Morrey and Campanato spaces. - Banach J. Math. Anal. 8, 2014, $221-268$.

[43] Liang, Y., and D. YANG: Musielak-Orlicz Campanato spaces and applications. - J. Math. Anal. Appl. 406, 2013, 307-322.

[44] Liang, Y., and D. YANG: Intrinsic square function characterizations of Musielak-Orlicz Hardy spaces. - Trans. Amer. Math. Soc. 367, 2015, 3225-3256.

[45] Liu, J., D. D. Haroske, and D. YANG: New molecular characterizations of anisotropic Musielak-Orlicz Hardy spaces and their applications. - J. Math. Anal. Appl. 475, 2019, 13411366 .

[46] Liu, L., D.-C. Chang, X. Fu, and D. YAng: Endpoint boundedness of commutators on spaces of homogeneous type. - Appl. Anal. 96, 2017, 2408-2433.

[47] Liu, L., D.-C. Chang, X. Fu, and D. YAng: Endpoint estimates of linear commutators on Hardy spaces over spaces of homogeneous type. - Math. Meth. Appl. Sci. 41, 2018, 5951-5984.

[48] Lu, S. Z.: Four lectures on real $H^{p}$ spaces. - World Scientific Publishing Co., Inc., River Edge, N. J., 1995.

[49] Macías, R. A., and C. Segovia: Lipschitz functions on spaces of homogeneous type. - Adv. Math. 33, 1979, 257-270.

[50] Macías, R. A., and C. Segovia: A decomposition into atoms of distributions on spaces of homogeneous type. - Adv. Math. 33, 1979, 271-309.

[51] Muckenhoupt, B., and R.L. Wheeden: Two weight function norm inequalities for the Hardy-Littlewood maximal function and the Hilbert transform. - Studia Math. 55, 1976, 279 294. 
[52] NakaI, E.: Pointwise multipliers on weighted BMO spaces. - Studia Math. 125, 1997, 35-56.

[53] Nakai, E., and K. Yabuta: Pointwise multipliers for functions of weighted bounded mean oscillation on spaces of homogeneous type. - Math. Japon. 46, 1997, 15-28.

[54] Reed, M., and B. Simon: Methods of modern mathematical physics. I. Functional analysis. Second edition. - Academic Press, Inc., [Harcourt Brace Jovanovich, Publishers], New York, 1980.

[55] Stein, E. M.: Singular integrals and differentiability properties of functions. - Princeton Univ. Press, Princeton N. J., 1970.

[56] Stein, E. M.: Harmonic analysis: Real-variable Mmthods, orthogonality, and oscillatory integrals. - Princeton Univ. Press, Princeton, N. J., 1993.

[57] Stein, E. M., and G. Weiss On the theory of harmonic functions of several variables. I. The theory of $H^{p}$-spaces. - Acta Math. 103, 1960, 25-62.

[58] Strömberg, J.-O., and A. Torchinsky: Weighted Hardy spaces. - Lecture Notes in Math. 1381, Springer-Verlag, Berlin, 1989.

[59] YAng, D., Y. Liang, and L. D. KY: Real-variable theory of Musielak-Orlicz Hardy spaces. Lecture Notes in Math. 2182, Springer-Verlag, Cham, 2017.

[60] YANG, D., and S. YANG: Local Hardy spaces of Musielak-Orlicz type and their applications. - Sci. China Math. 55, 2012, 1677-1720.

[61] YANG, D., and Y. ZHOU: Radial maximal function characterizations of Hardy spaces on RDspaces and their applications. - Math. Ann. 346, 2010, 307-333.

[62] YAnG, D., and Y. Zhou: New properties of Besov and Triebel-Lizorkin spaces on RD-spaces. - Manuscripta Math. 134, 2011, 59-90.

Received 30 January 2019 • Accepted 7 June 2019 